\title{
A comparison of the growth and asexual reproduction by Cryphonectria parasitica isolates infected with hypoviruses via anastomosis and transfection
}

Jenise M. Bauman

West Virginia University

Follow this and additional works at: https://researchrepository.wvu.edu/etd

\section{Recommended Citation}

Bauman, Jenise M., "A comparison of the growth and asexual reproduction by Cryphonectria parasitica isolates infected with hypoviruses via anastomosis and transfection" (2005). Graduate Theses, Dissertations, and Problem Reports. 2298.

https://researchrepository.wvu.edu/etd/2298

This Thesis is protected by copyright and/or related rights. It has been brought to you by the The Research Repository @ WVU with permission from the rights-holder(s). You are free to use this Thesis in any way that is permitted by the copyright and related rights legislation that applies to your use. For other uses you must obtain permission from the rights-holder(s) directly, unless additional rights are indicated by a Creative Commons license in the record and/ or on the work itself. This Thesis has been accepted for inclusion in WVU Graduate Theses, Dissertations, and Problem Reports collection by an authorized administrator of The Research Repository @ WVU. For more information, please contact researchrepository@mail.wvu.edu. 


\title{
A comparison of the growth and asexual reproduction by Cryphonectria parasitica isolates infected with hypoviruses via anastomosis and transfection
}

\author{
Jenise M. Bauman
}

Thesis submitted to the Davis College of Agriculture, Forestry, and Consumer Sciences at West Virginia University in partial fulfillment of the requirements for the degree of

\author{
Master of Science \\ in \\ Plant Pathology \\ William L. MacDonald, Ph.D., Chair \\ John R. Brooks, Ph.D. \\ James B. Kotcon, Ph.D. \\ Daniel G. Panaccione, Ph.D. \\ Division of Plant and Soil Sciences \\ Morgantown, West Virginia \\ 2005
}

Keywords: hypovirulence, biological control, chestnut blight, Cryphonectria parasitica Copyright 2005 Jenise M. Bauman 


\begin{abstract}
A comparison of the growth and asexual reproduction by Cryphonectria parasitica isolates infected with hypoviruses via anastomosis and transfection
\end{abstract}

Jenise M. Bauman

Transfection is an alternative laboratory method of hypovirus transmission that can be used to create hypovirulent strains. This technique involves the insertion of a synthetic hypovirus dsRNA into individual fungal spheroplasts by electroporation. A transfected strain has the same phenotypic traits as a strain infected via anastomosis. Results from a preliminary study at West Virginia University in 1998-99 indicated that recovery of hypovirulent isolates was similar between CHV1-infected anastomosed and transfected isolates. The objective of this research was to compare the two hypovirus acquisition methods in three experiments. The first study confirmed the performance of anastomosed and transfected isolates tested in a 1998-99 field study. In addition to the isolates previously evaluated, a third set of isolates containing CHV3-County Line was included. The size of cankers and the reisolation of $\mathrm{V}$ and $\mathrm{HV}$ isolates were evaluated. The second study evaluated stroma production and hypovirus transmission to conidia. The third study involved laboratory tests to evaluate hypovirus transmission to different vegetative incompatible isolates of $C$. parasitica via anastomosis. The field study site, located in the Monongahela National Forest, utilized 96 American chestnut trees. Four inoculations per tree were established to assess hypovirulent isolates while two inoculations per tree were used for virulent isolates. After 5 and 12 months, all cankers were measured to determine growth rates of the isolates. To evaluate the recovery of $\mathrm{V}$ and $\mathrm{HV}$ isolates, three bark samples were collected from the margin of each canker using a bone marrow biopsy instrument. Bark samples were cultured, and resulting colonies were analyzed for morphology to determine if the isolates retrieved were similar to those used to initiate the cankers. To evaluate stroma production, all cankers were observed and scored on a subjective $0-3$ scale for the production of pycnidia. To evaluate the production of hypovirulent inoculum in vitro, conidia produced in Petri dishes were single spored on PDA. Germinating spores were observed for hypovirulent morphology to determine the influence of the isolate and the infection method on the production of hypovirulent conidia. Results evaluating size of canker were comparable to the 1998-99 study. These results showed that no differences existed in the size of cankers produced when isolates were infected either by anastomosis or transfection. When reisolated, there was no clear trend observed in either anastomosed or transfected isolates with regard to hypovirus retention. Very few stroma were produced after one year in the field from either transfected or anastomosed hypovirulent isolates. The single spore results showed high HV transmission rates among most isolates regardless of virus acquisition in vitro. The results from the hypovirus transmission study showed virus acquisition by incompatible isolates was dependent on the alleles at the vic loci of the donor. The results from the entire study illustrated that the mode by which isolates were infected with hypovirus generally did not influence the behavior of an isolate; rather differences observed were more dependent on the isolate and the hypovirus it harbored. 
Table of Contents

Acknowledgments........................................................... iii

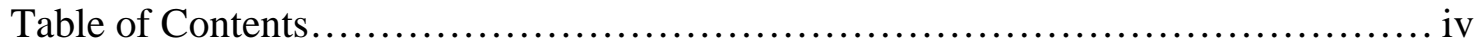

List of Tables..................................................................

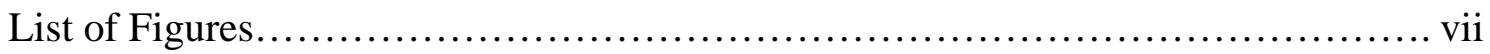

List of Appendices......................................................... ix

Introduction..................................................................

Literature Review.............................................................

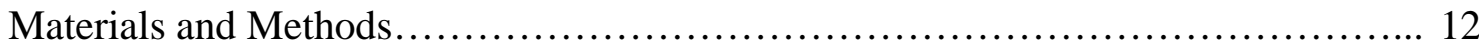

Test Strains...................................................... 12

Field Plot Location and Description.................................... 15

Field Canker Initiation.............................................. 16

Field Data Collection................................................ 17

HV Recovery......................................................... 18

Analysis of In Vitro Asexual Sporulation by the Test Isolates................ 19

Analysis of In Vivo Asexual Sporulation by the Test Isolates................20

Conformation of Infecting Strain by VC Testing........................ 21

Horizontal Transmission by Vegetative Incompatible Isolates............... 22

Statistical Analysis..................................................23

Results..................................................................... 25

Analysis of Canker Growth......................................... 25

Bark Sample Analysis to Evaluate Hypovirus Persistence................. 31

Canker Stroma Rating............................................. 38

Comparison of HV Transmission to Conidia in vivo.................................. 39

Comparison of HV Transmission to Conidia in vitro........................ 44

Hypovirus Transmission via Anastomosis in vitro........................................ 46

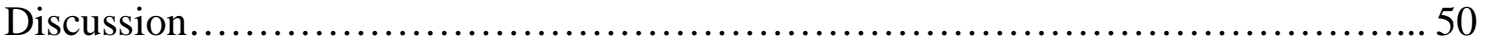

Summary............................................................. 65

References............................................................... 66

Appendices................................................................ 69 


\section{List of Tables}

Table 1. Test isolates of Cryphonectria parasitica. The "+" denotes the presence of hypovirulent isolates. "NA" indicates that the data is not applicable because isolates

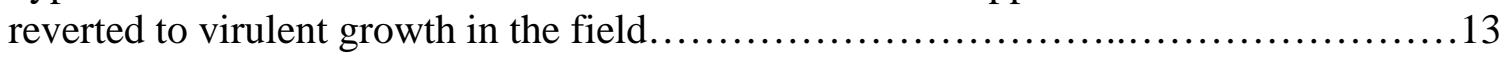

Table 2. Tukey's HSD test contrasting size of canker produced by the virulent isolates measured after 5 months (11/12/03) and after 12 months (7/29/04). Canker sizes produced by isolates designated by the same letter are not significantly different..................................................................26

Table 3. Tukey's HSD test used to contrast average sizes $(L+W / 2)$ in centimeters of cankers produced by CHV1-Euro7 infected isolates recorded $5(11 / 12 / 03)$ and 12 months $(7 / 29 / 04)$ after initiation. Measurements in bold text indicate significant differences between anastomosed and transfected isolates. Isolates connected by the same letter are not significantly different.

Table 4. Tukey's HSD test used to contrast average sizes $(L+W / 2)$ in centimeters of cankers produced by CHV1-Ep713 infected isolates recorded 5 (11/12/03) and 12 months (7/29/04) after initiation. Measurements in bold text indicate significant differences between anastomosed and transfected isolates. Isolates connected by the same letter are not significantly different. ...

Table 5. Tukey's HSD test used to contrast average sizes $(\mathrm{L}+\mathrm{W} / 2)$ in centimeters of cankers produced by CHV3-County Line infected isolates recorded 5 (11/12/03) and 12 months (7/29/04) after initiation. Isolates connected by the same letter are not significantly different.

Table 6. Tukey's HSD test contrasting \% HV recovered from cankers produced by CHV1-Euro7 infected isolates sampled after 5 (11/12/03) and 12 months (7/29/04). Bold text indicates significant differences between anastomosed and transfected isolates. Means designated by the same letter are not significantly different.

Table 7. Tukey's HSD test contrasting \% HV recovered from cankers produced by CHV1-Ep713 infected isolates sampled after 5 (11/12/03) and 12 months (7/29/04). Bold text indicates significant differences between anastomosed and transfected isolates. Means designated by the same letter are not significantly different....................35 Table 8. Tukey's HSD test contrasting \% HV recovered from cankers produced by anastomosed CHV3-County Line-infected isolates sampled after 5 (11/12/03) and 12 months (7/29/04). Means designated by the same letter are not significantly different....37

Table 9. Average stroma rating and percentage of HV transmission to conidia from CHV1-Euro7 isolates rated and sampled after 5 (11/12/03) and 12 months (7/29/04)...41

Table 10. Average stroma rating and percentage of $\mathrm{HV}$ transmission to conidia from CHV1-Ep713 isolates rated and sampled after 5 (11/12/03) and 12 months (7/29/04)...42 Table 11. Average stroma rating and percentage of $\mathrm{HV}$ transmission to conidia from CHV3-County Line isolates rated and sampled after 5 (11/12/03) and 12 months

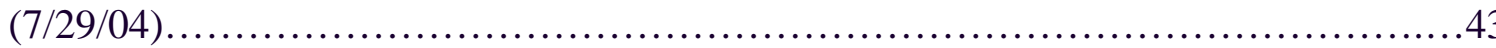

Table 12. Tukey's HSD test comparing the percentage of HV transmission to progeny between CHV1-Euro7 and CHV1-Ep713 anastomosed and transfected isolates. Means designated by the same letter are not significantly different. No significant differences existed between anastomosed and transfected isolates. 
Table 13. Tukey's HSD test comparing CHV3-County Line infected isolates. The percentage of $\mathrm{HV}$ transmission to progeny among parental backgrounds when single conidia were isolated from cultures grown on PDA was measured. Means designated by the same letter are not significantly different.....................................46 Table 14. Results for horizontal transmission of virulent isolates (top row) when paired with anastomosed (A) and transfected (T) HV isolates (left hand column). Number reported is the number of times the virulent isolate was converted via anastomosis out of 5 pairings. NA= Not available for this study. 48

Table 15. Chi-square test contrasting frequency of hypovirus transmission to incompatible isolates infected with CHV1-Euro7 or CHV1-Ep713. Isolates connected by the same letter are not significantly different. Number of conversion (\# Conv) are out of

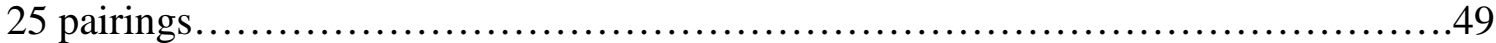

Table 16. Chi-square test contrasting horizontal transmission between vegetative incompatible isolates infected with CHV3-County Line. Isolates connected by the same letter are not significantly different. Number of conversion (\# Conv) are out of 25 pairings. 


\section{List of Figures}

Figure 1. Six different virulent Cryphonectria parasitica isolates used in this study....11 Figure 2. Examples of hypovirus expression among three hypoviruses:

Schomberg(CHV1-Euro7), WR2(CHV1-Ep713), and Euro7ssv(CHV3-County Line)

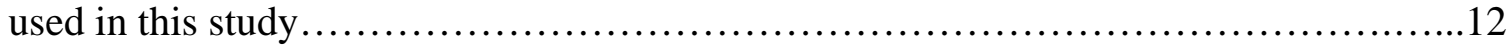

Figure 3: Petri dish containing one HV isolate and one compatible virulent isolate paired for anastomosis-mediated transfer of hypovirus dsRNA............................14

Figure 4. Satellite photo (left) and topographic map (right) illustrating the location of

this field study. Red arrows indicate the location of the plots.........................15

Figure 5. Seven mm cork borer (left) used to create wound for canker initiation. Bark

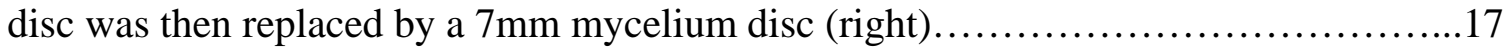

Figure 6. Sampling scheme for $2 \mathrm{~mm}$ bark plugs................................ 18

Figure 7. Colonies germinating from bark plugs on GYE (left). Resulting colonies that were transferred from GYE and cultured on PDA (right) ............................19

Figure 8. Single asexual spores (conidia) that have germinated on PDA. Four of the five resulting colonies are hypovirus-infected and exhibit the altered growth habit of a hypovirulent isolate...........................................................20

Figure 9. Seven mm bark disc removed from canker (left). Pycnidia containing conidia

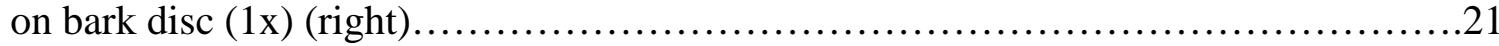

Figure 10. Schematic placement of test isolates on Brom Cresol Green medium (left). Seven-day-old cultures on Brom Cresol Green medium illustrating barrage formation (right).

Figure 11. Barrage formation appears as a distinct line indicating horizontal transmission has been prevented (left). Red arrow denotes change in virulent morphology indicating horizontal transmission of hypovirus has occurred (right).......23 Figure 13. Average canker size produced by isolates measured November 12, 2003 and July 29, 2004 without regard to hypovirus or background. No statistical difference in the size of canker produced by anastomosed or transfected isolates existed when compared at either sample periods

Figure 14. Average canker area produced by all anastomosed isolates when cankers were measured after 5 (11/12/03) and 12 months (7/29/04). Cankers produced by CHV3County Line-infected isolates were significantly smaller.

Figure 15. Comparison of the percentage of parental hypovirulent (HV), virulent (V), and non-C. parastica (Non Cp) isolates without regard to hypovirus or fungal host. Bark plugs were recovered from cankers after $5(11 / 12 / 03)$ and 12 months (7/29/04). There was no significant differences between anastomosed and transfected isolates............32 Figure 16. Comparison of CHV3- County Line, CHV1-Euro7, and CHV1-Ep713 hypoviruses in terms of percentage of hypovirulent (HV), virulent (V), and non- $C$. parastica (Non Cp) isolates recovered from all cankers initiated by anastomosed isolates

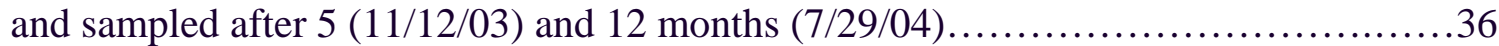
Figure 17. Comparison of stroma ratings (0-3) produced by cankers initiated by anastomosed and transfected isolates evaluated after $5(11 / 12 / 03)$ and 12 months (7/29/04). There is no statistical difference between anastomosed and transfected isolates. 
Figure 18. The percentage of hypovirulent (HV) and virulent (V) colonies that resulted from pycnidia produced by anastomosed and transfected isolates, regardless of hypovirus or parental background, sampled after $5(11 / 12 / 03)$ and 12 months $(7 / 29 / 04) \ldots \ldots \ldots . .40$ Figure 19. Average stroma produced by all anastomosed isolates when cankers were rated after 5 (11/12/03) and 12 months (7/29/04). CHV1-Euro7 infected isolates produced significantly more stroma in November than CHV1-Ep713 or CHV3County Line.....................................................................

Figure 20. Percentage of hypovirulent (HV) and virulent (V) colonies resulting from single-conidial isolations from anastomosed and transfected isolates, regardless of hypovirus and parental background, cultured in vitro................................44

Figure 21. Comparison of the percentage of hypovirulent (HV) and virulent (V) colonies resulting from single-conidial isolation from anastomosed isolates cultured in vitro.

CHV3-County Line isolates had a significantly lower transmission rates when compared to CHV1-Euro7 and CHV1-Ep713...........................................46

Figure 22. The percentage hypovirus transmission that resulted from pairing incompatible anastomosed and transfected isolates, regardless of hypovirus or parental background. There were no differences between anastomosed and transfected isolates 


\section{List of Appendices}

Appendix A: Media Used............................................... 69

Appendix B: Peptone Solution.......................................... 71

Appendix C: dsRNA Extraction Protocol..................................... 71

Appendix D: Tree number and placement of hypovirulent isolates............... 73

Appendix E: Figures 23-28. Percentage of hypovirulent (HV), virulent (V), and non-C. parastica (Non Cp) isolates recovered from cankers initiated by all isolates/hypovirus combinations sampled after $5(11 / 03)$ and 12 months .......................76-82 Appendix F: ANOVA Tables...........................................83-91 
Introduction

The impact of chestnut blight, caused by the pathogen Cryphonectria parasitica, has diminished in Europe presumably due to a natural biological control caused by hypovirus infection. Hypoviruses are virus particles of dsRNA found in the cytoplasm of debilitated strains of $C$. parasitica. Although infectious, hypovirus transmission is only known to occur in nature via anastomosis among vegetatively compatible strains. Hypovirus dissemination with subsequent diminishment of fungal virulence has presumably resulted in the "spontaneous healing" of blighted chestnuts in European forests and orchards.

Hypovirulence-mediated biological control has been far less successful in North America presumably due to barriers caused by diversity in vegetative compatibility among C. parasitica strains. Furthermore, the superficial cankers caused by hypovirulent isolates do not produce the amount of conidia to rival the wild-type strains. A field isolate with the ability to persist and produce hypovirulent inoculum appears to be paramount for the success of biological control. Studies analyzing the fungal genome have been conducted with one of the intentions of selecting isolates that display a less debilitated growth habit after hypovirus infection (Elliston 1977, Chen and Nuss, 1999). Anastomosis-mediated transfer of hypovirus dsRNA is commonly used to study a variety of fungal genomes. When a HV donor is vegetatively incompatible with the $\mathrm{V}$ recipient, infection via anastomosis can be time consuming if at all achievable. Transfection is a laboratory technique used to overcome the limitations caused by vegetative incompatibility. A full-length synthetic coding strand transcript is introduced into the spheroplasts by eletroporation and surviving spheroplasts are then regenerated on 
specialized media. This has proven to be a versatile method capable of extending hypovirus infection into a variety of VC types as well as other fungal species related to $C$. parasitica (Chen and Nuss 1999).

Previous studies on excised stems (Chen and Nuss 1999) and chestnut sprouts in the field (Double, unpublished data) have concluded that transfected isolates behave analogous to their anastomosed counterparts in terms of pathogenicity and HV persistence. The objective of this study was to extend on the previous analyses comparing anastomosed to transfected isolates. Pathogenicity and HV persistence were retested and other variables were added such as stroma production in the field, hypovirus transmission to conidia (in vivo and in vitro), and HV conversion of incompatible isolates. Six isolates representing different parental backgrounds and three different hypoviruses were used to access what influence the genome of the fungus, genome of the hypovirus, and method of infection has on an isolates potential as a biological control agent. 


\section{Literature Review}

American chestnut (Castanea dentata) was eliminated as a canopy tree from eastern forests with the introduction of Cryphonectria parasitica into New York City in the early 1900's. C. parasitica is a fungal pathogen whose origin has been linked to nursery stock imported into the United States from Asia (Griffin, 1986). American chestnut, valued for its economical and ecological qualities, was highly susceptible to this canker inciting pathogen. Most conditions favored the pathogen including two abundantly produced spore types that provided efficient means of spread (Kuhlman, 1978). Conidia were disseminated by rain, insects, and animals and the ascospores were wind blown over long distances. The fungus enters through wounds in bark and produces a canker that kills the cells in the cambium region through the action of enzymes produced by the invading mycelial fans (Hebard et al., 1984).

American chestnut occupied, on average, twenty-five percent of the eastern forest and in certain forest stands comprised more than seventy percent (Braun, 1950). A prolific host coupled with the high susceptibility to the blight was responsible for the epidemic. Once the devastation was evident, a variety of strategies were pursued to control the pathogen. Control measures included eradication of infected tissue and trees, treatment with Bordeaux mixture, and quarantine of infected hosts (Kuhlman, 1978). None of these control measures were successful. As a result, breeding programs were initiated in the 1930's to incorporate resistant genes from Chinese and Japanese chestnut into American chestnut. However, the hybrids that resulted were either deficient in adequate field resistance or they lacked the American chestnut characteristics. By the 1950's, 200 million acres of American chestnut from Maine to Georgia had succumbed to 
the disease. This once prominent species that measured up to one-hundred feet in height was relegated to a minor place in the eastern forests (Kuhlman, 1978). Fortunately, American chestnut was saved from extinction by its ability to sprout from surviving root systems (MacDonald and Fulbright, 1991). However, the fungus generally girdles the sprouts before they reach a size of any significance.

The European chestnut (Castanea sativa) was similarly affected by the accidental introduction of C. parasitica into Italy in 1938. The disease devastated the Italian forests and orchards. In the 1950's, less than twenty years after the onset of the epidemic, Antonio Biraghi, an Italian plant pathologist, observed “spontaneous healing” in many of the abandoned chestnut orchards. Biraghi observed regenerating European chestnuts and hypothesized that this change either was due to the loss of pathogenicity in the fungus or the development of partial resistance in the host (Mittempergher, 1978).

Jean Grente, a French mycologist in the 1960's, agreed with Biraghi's hypothesis that an alteration in the fungus resulted in the spontaneous recovery of European chestnut (Mittempergher, 1978). Grente's research consisted of numerous isolations from cankers that revealed a culture with an altered fungal growth habit, reduced sporulation, and less pigmention. These isolates also had a reduced pathogenicity in vivo, and as a result they were referred to as "hypovirulent". Grente demonstrated the transmissible nature of hypovirulence by pairing both strains, in vitro, and observing the conversion of the virulent strain to the hypovirulent form following their anastomosis (Grente and Berthelay-Sauret, 1978). Grente intimated that anastomosis allows for cytological determinants of hypovirulence to be exchanged between the two thalli (Grente and 
Berthelay-Sauret, 1978). Conversion, however, did not occur in some situations when anastomosis failed due to the phenomenon called vegetative incompatibility.

Some researchers hypothesized that the debilitated nature of the European cankers was caused by a fungal virus (Day et al., 1977). In the late 1970's, double-stranded RNA (dsRNA) was identified as the genetic material associated with virus-like particles in hypovirulent isolates (Dodds, 1978). The presence of the dsRNA was detected from European and American hypovirulent isolates via gel electrophoresis by phenol/chloroform extractions that show the presence of dsRNA bands along a gel gradient. Variations in banding patterns of dsRNA molecules suggested hypovirulent isolates were not all identical and several viruses may be responsible for debilitation of the host. These different forms may be distinguished by their degree of pathogenicity to C. parasitica and their effects on the morphology of the fungal colony (Day, 1978). Gel electrophoresis provided the first evidence of virus-like particles by a purified extract of pleomorphic, club-shaped particles from a French hypovirulent strain (Dodds, 1980). This finding confirmed the cytoplamic factor by detecting dsRNA following anastomosis of an isolate that was previously dsRNA free (Dodds, 1980).

The first observation of virus-like particles in the cytoplasm of European and American hypovirulent isolates was made by transmission electron microscopy (Newhouse et al., 1983). Description of similar cytoplasmic features between the virulent and hypovirulent isolates were documented. However, spherical., lipidencapsidated virus-like particles were found only in the hypovirulent strains of both European and American isolates and absent in the virulent strains. Electron microscopy also provided ultrastructural evidence of cytoplasmic continuity between compatible 
virulent and hypovirulent isolates by observing fusion at an anastomosis bridge

(Newhouse et al., 1983). This bridge was not evident between two incompatible isolates; thus reinforcing the idea that vegetative incompatibility is a barrier to virus transmission (Newhouse et al., 1983).

The infectious nature of the dsRNA was identified in the 1990's (Nuss, 1992). Research initially involved direct analysis of dsRNA to identify its structural properties, cloning sequence analysis and in vitro expression to determine the genetic organization. Understanding of the genetic organization of the hypovirus eventually allowed researchers to introduce cDNA copies of portions of the viral genome into the nucleus of virulent $C$. parasitica via a cDNA mediated-transformation. This genetic manipulation resulted in a fungal colony that exhibited the hypovirulent phenotype. When paired with a compatible virulent isolate, the hypovirulent engineered strain converted a virulent isolate to a hypovirulent form following anastomosis. These results provided direct evidence that a viral-like dsRNA is the genetic determinate responsible for transmissible hypovirulence in the chestnut blight fungus (Chen and Nuss, 1999).

Cloning sequence analysis has allowed for closer examination of dsRNA in the respect to the amount, size, and number of segments that often differ among virusinfected isolates. A few of the dsRNAs associated with hypovirulence in North America and Europe have been examined for nucleotide sequence relatedness at the molecular level (Paul and Fulbright, 1988). As a result, four types of dsRNA viruses have been described and placed in the genus Hypovirus in the family Hypoviridae (Hillman et al., 1994). Those hypoviruses are referred to as CHV1, CHV2, CHV3, and CHV4 based on the similarity of the cDNA sequence information (Hillman and Suzuki, 2004). 
CHV1 has been released in many areas of North America but never found naturally. Isolate Ep713 is a CHV1-type hypovirus containing isolate from Europe. The $12.7 \mathrm{~kb}$ Ep713 genome is comprised of two coding regions designated as ORF A and ORF B (Hillman et al., 1994). When compared to CHV1-Euro7, another European CHV1-type isolate, the level of nucleotide identity for the entire 5' non-coding domain is 93\%; but the CHV1-Euro7 genome is 11 nucleotides shorter than the CHV1-Ep713 genome (Chen and Nuss, 1999). Despite the similarity, these two isolates have phenotypic and morphological traits that differ. CHV1-Ep713 is a debilitated isolate in vitro and produces small cankers and few asexual spores in vivo. In contrast, CHV1Euro7 grows rapidly in vitro and produces larger cankers in vivo with moderate asexual sporulation (Chen and Nuss, 1999).

CHV3-type isolates are found in North America. Biological control with this hypovirus occurs naturally in chestnut stands in Michigan (Milgroom and Cortesi, 2004). CHV3-Grand Haven 2 (GH2) is an example of this hypovirus and is comprised of one ORF and a 9.8 kb genome (Hillman and Suzuki, 2004). Phenotypic traits differ considerably in CHV3 infected isolates. CHV3-GH2 infected isolates are severely debilitated. These isolates produce small, superficial cankers both in vivo and in vitro.

Despite the variation among hypovirus-containing strains, they all have the potential to arrest canker expansion, as noted in the callousing cankers in European orchards and in North America. When C. parasitica strains are infected with a hypovirus, the chestnut tree has the opportunity to activate its defenses and produce callus tissue in sufficient quantities to contain the infection. Chestnut blight has 
diminished in Europe, presumably due to a natural biological control caused by hypoviruses (Chen and Nuss, 1999).

Hypovirulence-mediated biological control has been far less successful in North America presumably due to barriers caused by diversity in vegetative compatibility in $C$. parasitica populations (Milgroom et al., 1991). Vegetative incompatibility restricts fungal anastomosis preventing the spread of the hypovirus. Consequently, a lack of anastomosis results in a lack of hypovirus dissemination thereby inhibiting biological control (Anagnostakis and Waggoner, 1981). There are sixty-four possible European vegetative types as indicated by six loci (Cortesi and Milgroom, 1998). Researchers suspect that there are over one hundred possible vegetative compatible types of the fungus in North America (Anagnostakis and Day, 1979). This diversity in eastern North America may preclude natural dissemination of hypoviruses in the forest. Tree survival is anticipated only when conversion of all cankers located on the main stem occur; failure to convert even one canker may result in the death of a tree. Viral acquisition by all cankers is therefore imperative for the success of biological control. Furthermore, the superficial cankers caused by hypovirulent isolates do not produce the amount of conidia to rival the wild-type strains. Therefore, a field isolate with the ability to produce hypovirulent inoculum is paramount for the success of biological control.

Studies that analyze the fungus have been conducted with intention of selecting isolates that display a less debilitated growth habit after hypovirus infection (Elliston 1977; Chen and Nuss, 1999). The notion is that an isolate that has a greater tolerance to hypovirus-infection will produce a larger canker with the propensity to sporulate asexually. Other characteristics that are sought after to promote a successful biological 
control agent include persistence in a forest-ecosystem as well as the ability to transmit hypovirus to a variety of vc types.

Anastomosis-mediated transfer of hypovirus dsRNA transmits hypovirus from an infected isolate to one that is virus-free and is commonly used to study a variety of fungal genomes and hypoviruses. When the donor and recipient isolates are vegetatively compatible, hypovirus transmission is easy to achieve. However, if incompatible, other methods must be applied. One technique entails pairing the donor and recipient fungi on Cellophane ${ }^{\circledR}$ that is cut to the diameter of 100x15 mm Petri plate. Agar plugs containing mycelium of the donor and the recipient are placed adjacent to one another at the perimeter of a Petri plate and replicated on 10 plates. Cultures are incubated at 20 degrees $\mathrm{C}$ and observed after 7 to 10 days for a change in the morphology of the recipient isolate.

Another method involves inoculations on sterilized excised stems. Two $7 \mathrm{~mm}$ bark discs are removed adjacent to one another on the excised stem. The bark disc is then replaced with a similar size piece of mycelium removed from the margin of seven-dayold cultures representing the donor and recipient isolate. Inoculations are replicated 5-10 times and incubated at room temperature in a moisture chamber for 7 to 10 days. Bark plugs are then removed from the margin of the recipient canker and plated onto GYE amended with antibiotics and transferred to PDA after 48 hours. Cultures are then incubated at $20^{\circ} \mathrm{C}$ for $7-10$ days and observed for HV morphology.

These methods of hypovirus acquisition tend to be time consuming if at all achievable. Transfection is a laboratory technique that can overcome the limitations caused by vegetative incompatibility. This technique involves the insertion of hypovirus 
synthetic transcripts into individual fungal spheroplasts by electroporation. To obtain the spheroplasts, hyphae are washed with cell degrading enzymes. Spheroplasts are then mixed with synthetic hypovirus dsRNA in an electrical conductive suspension. Electroporation is accomplished by exposing spheroplasts to $1.5 \mathrm{kV}$ and 200 ohms of electricity (Chen et al., 1996). This forces the synthetic dsRNA into the pores of the spheroplasts which can then be regenerated on specialized media. Successfully transfected colonies can be selected based on phenotype. Resulting transfected strains have phenotypic traits identical to the parent strain. This technique demonstrates that a synthetic transcript, without the other organelles transferred in during anastomosis, can initiate an infection when introduced into fungal spheroplasts (Chen et al., 1994). Transfection thus overcomes the barriers imposed by vegetative incompatibly.

This has proven to be a versatile method capable of extending hypovirus infection into a variety of VC types as well as to other fungal species related to C. parasitica (Chen and Nuss 1999). Previous studies assessing the pathogenicity of these HV isolates on excised stems have concluded that transfected isolates are comparable to their anastomosed counterparts in terms of size of canker and sporulation (Chen and Nuss 1999). A 1998 field study conducted at West Virginia University compared transfected and anastomosed isolates with regard to their pathogenicity and HV persistence. Isolates used in this study included six virulent strains each infected with one of two different hypoviruses (CHV1-Euro7 and CHV1-Ep713). One set of six isolates had acquired the hypovirus through anastomosis and the other by transfection. Results indicated that after 16 months the two methods of hypovirus acquisition produced cankers that were comparable in size. When bark samples were extracted from each canker there was no 
difference in recovery of infecting isolate whether the canker was initiated by an anastomosed or a transfected isolate (Double, unpublished data).

The objective of this study was to extend the previous analyses comparing anastomosed to transfected isolates. Pathogencicity and HV persistence were retested and other variables were added such as stroma production in the field, vertical transmission to conidia (in vivo and in vitro), and horizontal conversion of incompatible isolates. In addition to the original isolates, a third set of isolates containing CHV3County Line were used. Thus, six different fungal isolates (parental backgrounds) and three different hypoviruses were used to evaluate the influence the method of infection, genome of the fungus and hypovirus had on an isolates potential as a biological control agent. 


\section{Materials and Methods}

\section{Test Strains}

The 36 strains of Cryphonectria parasitica that were used in this study are listed in Table 1. All virulent strains originally were isolated from natural cankers found on American chestnut with the exception of Euro7ssv. This virulent isolate originated from a single conidial isolation of hypovirulent isolate Euro7. The original HV isolate was obtained from a canker on European chestnut growing near Florence, Italy. The Bockenhauer and Schomberg virulent isolates were recovered from cankers on American chestnut growing near West Salem, WI. JR10 and WR2 were isolated from cankers in Front Royal., VA (Double, per. com.). Ep155 was first isolated in Bethany, CT (Anagnostakis, per. com.). These six unique virulent strains formed the basis for the isolate families (Figure 1). Each family consisted of one virulent isolate (V) and isogenic isolates that contained either: 1) CHV1-Euro7 or CHV1-Ep713 hypovirus acquired by anastomosis; 2) CHV1-Euro7 or CHV1- Ep713 hypovirus acquired by transfection; or 3)

CHV3-County Line hypovirus acquired by anastomosis (Figure 2, Table 1).

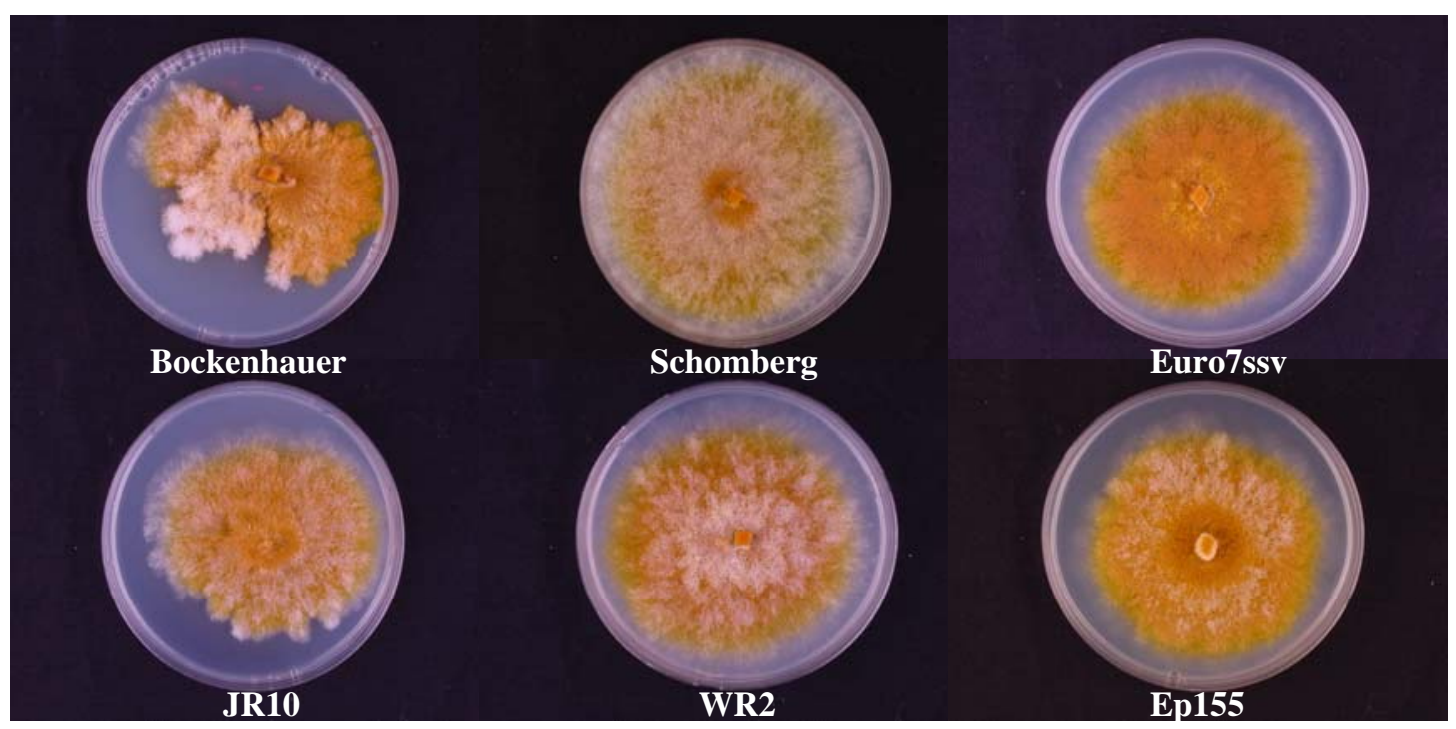

Figure 1. Six different virulent Cryphonectria parasitica isolates used in this study. 


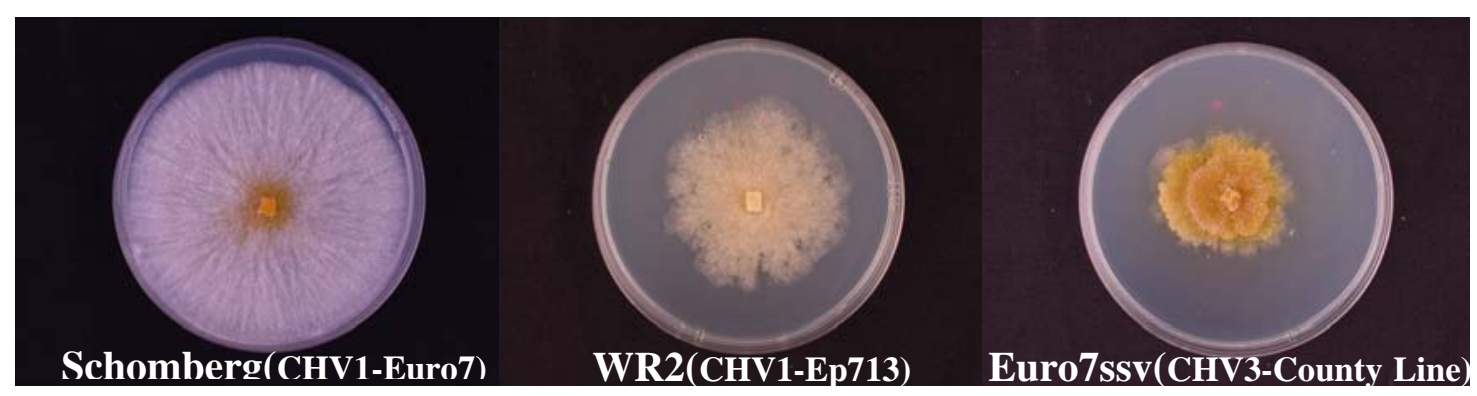

Figure 2. Examples of hypovirus expression among three hypoviruses:

Schomberg(CHV1-Euro7), WR2(CHV1-Ep713), and Euro7ssv(CHV3-County Line) used in this study.

\begin{tabular}{|c|c|c|c|c|c|c|}
\hline \multicolumn{7}{|c|}{ Parental Background } \\
\hline Hypovirus & Bockenhauer & Schomberg & Euro7ssv & JR10 & WR2 & Ep155 \\
\hline $\begin{array}{c}\text { CHV1-Euro7 } \\
\text { Anastomosed }\end{array}$ & + & + & + & + & + & NA \\
\hline $\begin{array}{c}\text { CHV1-Euro7 } \\
\text { Transfected }\end{array}$ & + & + & + & + & + & NA \\
\hline $\begin{array}{c}\text { CHV1-Ep713 } \\
\text { Anastomosed }\end{array}$ & + & + & + & NA & + & + \\
\hline $\begin{array}{c}\text { CHV1-Ep713 } \\
\text { Transfected }\end{array}$ & + & + & + & + & NA & + \\
\hline $\begin{array}{c}\text { CHV3- } \\
\text { County Line } \\
\text { Anastmosed }\end{array}$ & + & + & + & + & + & + \\
\hline $\begin{array}{c}\text { Virulent } \\
\text { (virus-free) }\end{array}$ & + & + & + & + & + & + \\
\hline
\end{tabular}

Table 1. Test isolates of Cryphonectria parasitica. The “+” denotes the presence of hypovirulent isolates. "NA" indicates that the data is not applicable because isolates reverted to virulent growth in the field.

\section{Anastomosed and Transfected Strains}

CHV1-Ep713 and CHV1-Euro7 were originally associated with isolates from superficial C. parasitica cankers on European chestnuts in Europe. CHV3-County Line hypovirus was found in a C. parasitica strain isolated from a superficial canker on American chestnuts in Michigan. Each anastomosed HV strain was created in the laboratory by anastomosis-mediated transfer of hypovirus dsRNA from infected isolates 
to the virulent parent. To accomplish this, a five-millimeter mycelial plug was transferred from the advancing margin of seven-to-ten day-old hypovirulent cultures and plated with each virulent isolate. Both mycelium plugs were placed adjacent to one another at the perimeter of a Petri plate (Figure 3) containing Difco® potato dextrose agar (PDA) amended with methionine and biotin (Appendix A). Cultures were incubated at 20 degrees $\mathrm{C}$. A change in the morphology of the virulent isolate indicated hypovirus transmission. All hypovirus transmissions were confirmed by phenol/chloroform extractions that show the presence of dsRNA bands along a gel gradient in the acquiring strain (Appendix C).

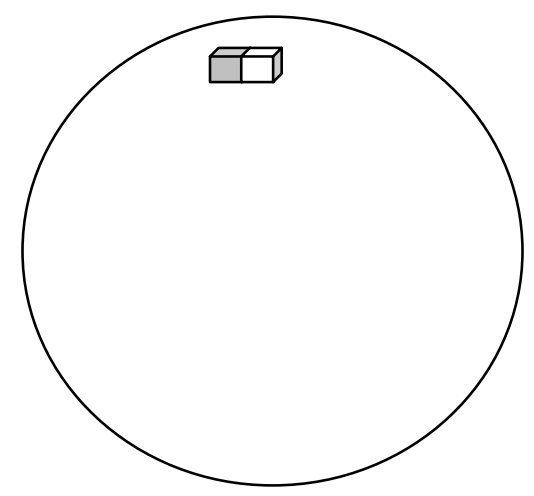

Figure 3: Petri dish containing one HV isolate and one compatible virulent isolate paired for anastomosis-mediated transfer of hypovirus dsRNA.

All transfected HV isolates were provided by Donald Nuss, Center for Biotechnology, Biosystems Research, University of Maryland Biotechnology Institute. Transfected isolates were created by electroporation of full-length synthetic copies of CHV1-Euro7 or CHV1-Ep713 coding strand transcripts into spheroplasts of virus-free strains as described by Chen et al., 1994. The spheroplasts were regenerated on specialized media and successful transfected colonies were selected based on phenotype. 
All isolates were cultured onto filter paper and stored at -20 degree C. When needed for study, a $5 \mathrm{~mm}$ piece of filter paper was transferred onto a Petri dish containing PDA. The isolates were then cultured at $20 \mathrm{C}, 58 \mathrm{~cm}$ beneath two 34 watt GE® "Wattmiser” fluorescent lamps with alternating 16 hours of light and 8 hours of darkness. Stock cultures were produced by transferring mycelium from the Petri dish to a test tube containing glucose yeast extract agar amended with antibiotics (Appendix A). All test tube slants were refrigerated at $5 \mathrm{C}$.

\section{Field Plot Location and Description}

The study site was located in the Potomac Ranger District of the Monongahela National Forest. The plot is on Big Mountain Road (USFS \#48) of the Snowy Mountain Quadrangle (Figure 4). The location was 3,670 feet above sea level with a global position of north $38^{\circ}, 35.5^{\prime}$ ', 58.6' /west $79^{\circ}, 35^{\prime \prime}, 25.5^{\prime}$. This area contained abundant sprout populations of American chestnut that ranged six to nine centimeters in diameter. Ninety-five blight-free American chestnut sprouts were used for the study. Each stem was marked with flagging and numbered in sequence with a yellow paint stick.
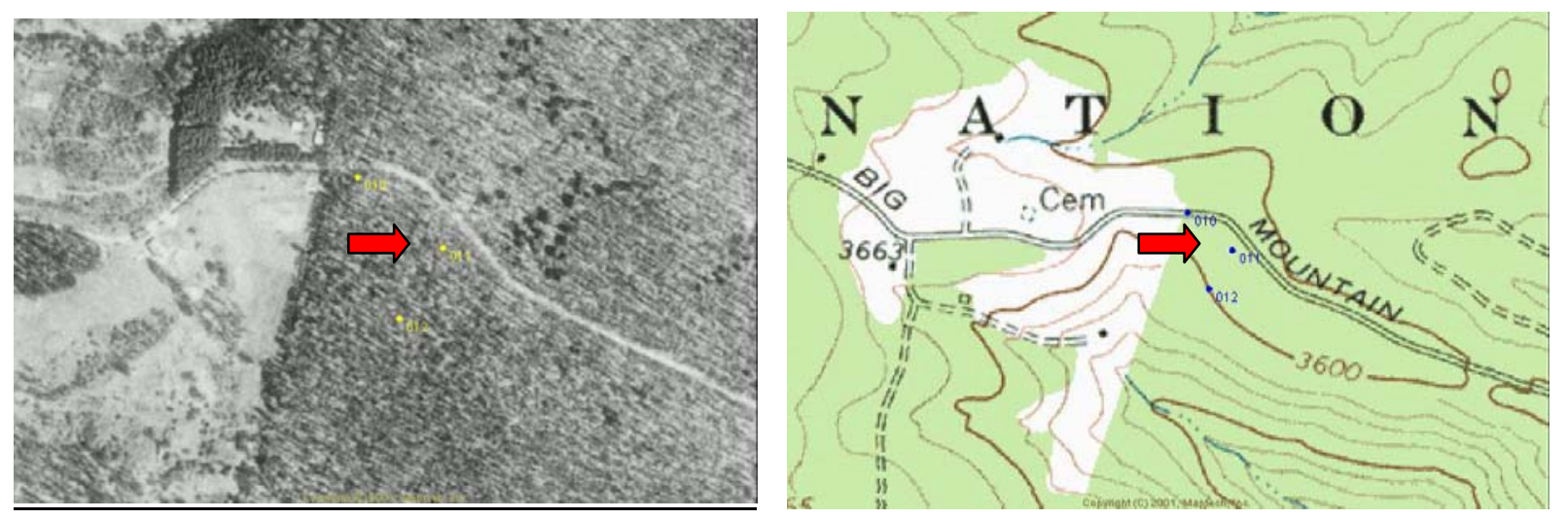

Figure 4. Satellite photo (left) and topographic map (right) illustrating the location of this field study. Red arrows indicate the location of the plots. 


\section{Field Inoculum}

Inoculum was prepared by transferring a small piece of mycelium from test tube slants to the center of Petri plates containing PDA medium. Three Petri plates of each isolate were established. Cultures were incubated for seven days at $20 \mathrm{C}$ under the light conditions previously described.

\section{Field Canker Initiation}

Hypovirulent and virulent cankers were initiated on July 15, 2003 by removing a 7-mm bark disk and inserting a similar size piece of mycelium removed from the margin of seven-day-old cultures (Figure 5). The inoculation site was covered with tape to retard desiccation. Four hypovirulent cankers were established per tree, at 50, 100, 150, and 200 centimeters above the ground on trees numbered 1 through 60 and 88 through 95 . To prevent more than one hypovirus hybridizing within the same thallus of a test isolate, only one hypovirus was designated per tree. For example, cankers on tree \# 1 were produced by isolates infected with CHV1-Ep713 and tree \# 4 hosted only those isolates infected with CHV1-Euro7. Tree number and isolate placement are listed in Appendix D. Trees designated to harbor the particular hypoviruses were selected at random. Once trees were assigned, canker placement and hypovirus-containing strains were assigned positions at random.

Virulent cankers were established two per tree at 50 and 150 centimeters above the ground on trees sixty-one through eighty-seven (Appendix D). To avoid hypovirus contamination of virulent cankers, the control trees were located 15 meters from the nearest hypovirus-inoculated tree, buffered by a logging road and existing forest trees. 
Tree number, the virulent parent, and canker placement were selected at random for trees designated as controls. All virulent and hypovirulent inoculations were replicated nine times for each isolate.
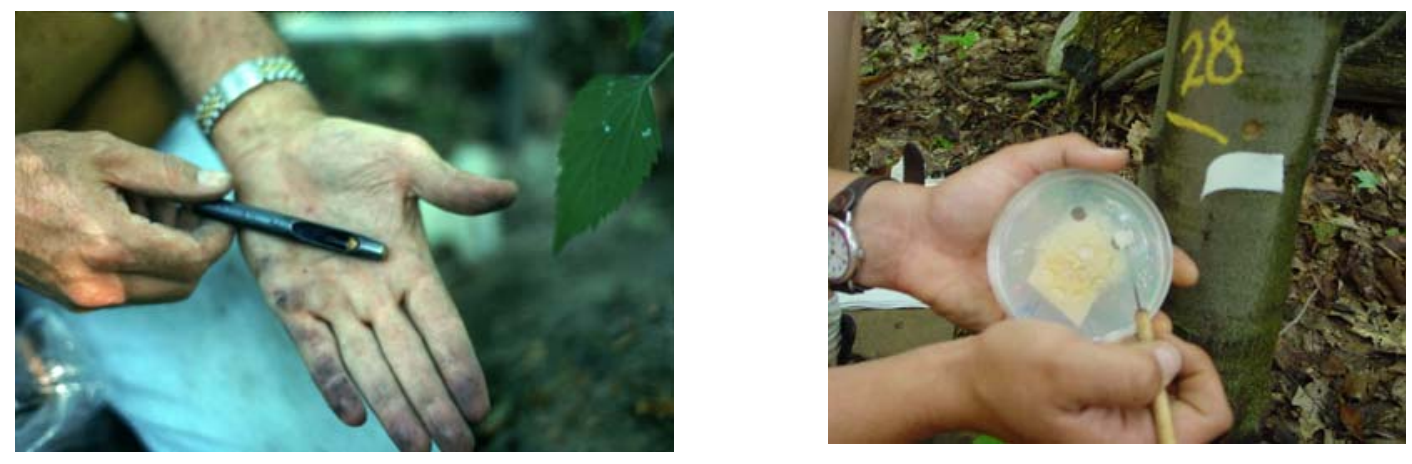

Figure 5. Seven-mm diameter cork borer (left) used to create wound for canker initiation. Bark disc was then replaced by a 7-mm diameter mycelium disc (right).

\section{Field Data Collection}

On November 12, 2003, the length and width of all cankers were measured $(\mathrm{cm})$ and their dimensions recorded to determine the growth rates of the isolates used in the study. All cankers were scored on a subjective scale (0-3) for the production of pycnidia ( 0 = no stromata; $1=$ slight stromata; 2 = moderate stromata; and, 3 = abundant stromata $)$. To assess whether the test isolate retained its original infection status in the canker, bark samples were collected. Three bark samples were removed from the margin of each canker with a bone marrow biopsy instrument as illustrated in Figure 6. Bark samples were stored in 96-well micro-titer plates (Fisherbrand Scientific) so that the position of the sample could be recorded. The wells were then covered with tape, returned to the laboratory and stored at- $20 \mathrm{C}$ until analysis. An identical bark plug collection was made on July 29, 2004. 


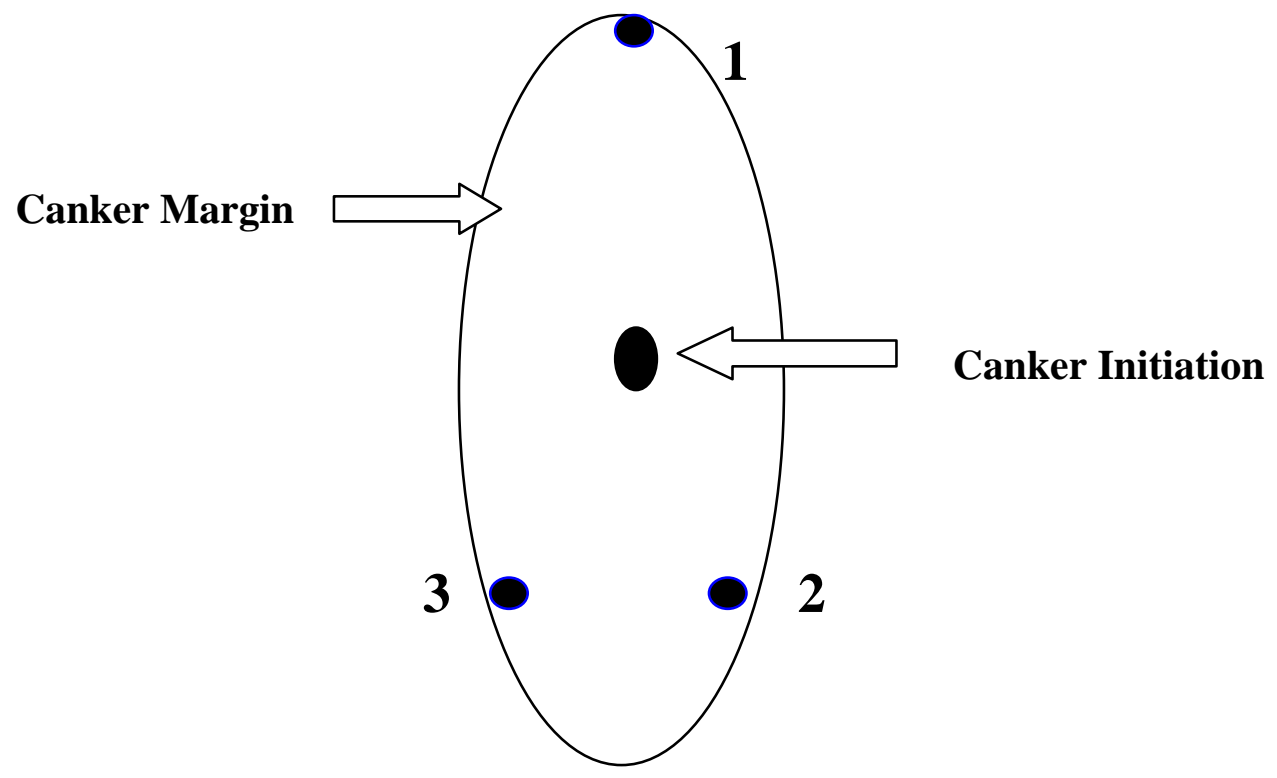

Figure 6. Sampling scheme for 2-mm bark plugs.

\section{HV Recovery}

In December 2003 and August of 2004, the two-millimeter bark samples from canker margins were removed from the freezer and allowed to thaw for one hour. Tape was removed from the microtiter plates, a nickel-plated wire mesh screen was attached, and the plates were submerged in a $0.6 \%$ sodium hypochlorite solution for 14 minutes. The solution was drained and bark plugs were transferred to a Petri dish containing glucose yeast extract medium (GYE) amended with antibiotics (Appendix A). Three samples per plate, placed approximately $45 \mathrm{~mm}$ apart, were incubated at room temperature under natural light conditions for five-to-seven days (Figure 7). The resulting mycelium was transferred to PDA and incubated at $20 \mathrm{C}$ with a 16:8 hour photoperiod for 7 to 10 days. The morphology of the resulting colonies was analyzed to 
determine if the retrieved isolates appeared hypovirulent or virulent and if they were similar to parental isolates used to initiate the cankers (Figure 7).

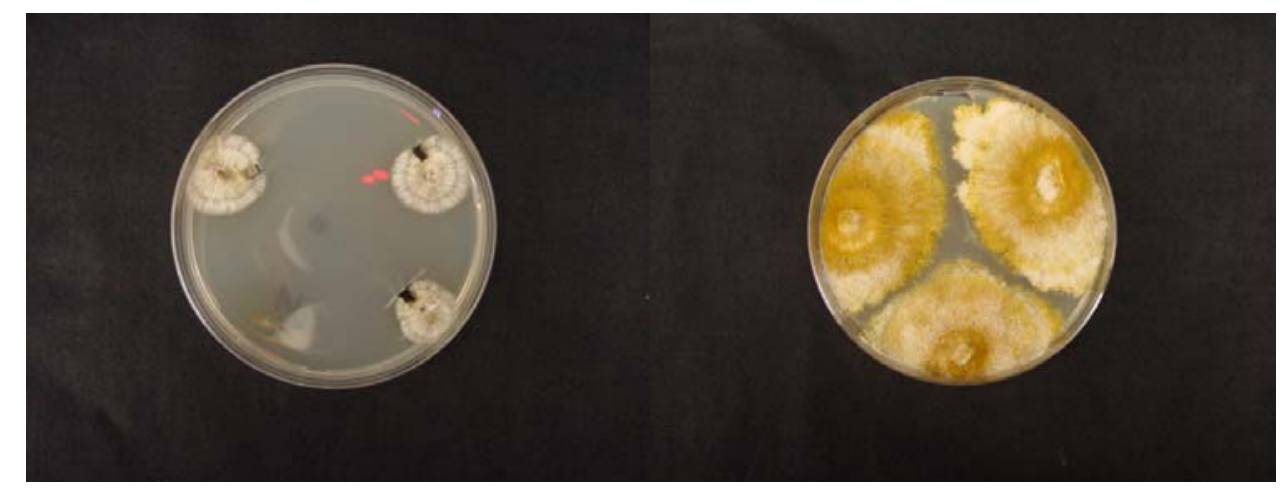

Figure 7. Colonies germinating from bark plugs on GYE (left). Resulting colonies that were transferred from GYE and cultured on PDA (right).

\section{Analysis of In Vitro Asexual Sporulation by the Test Isolates}

Prior to testing isolates in the field, single conidial cultures were obtained from isolates in vitro to establish the rate of hypovirus transmission to asexual progeny. To accomplish this, conidia were removed from the surface of 14-day-old cultures using a sterilized dissecting spear. A small amount of conidial exudate was collected on the spear tip and placed in $9 \mathrm{ml}$ of sterile $0.1 \%$ peptone solution (Appendix B). The spore suspension $(0.25 \mathrm{ml})$ was serially diluted to extinction. Spore dilutions of $10^{\overline{2}}$ for $\mathrm{HV}$ isolates and $10^{-5}$ for the $\mathrm{V}$ isolates were aseptically pipetted onto the surface of GYE amended with antibiotics and distributed evenly with a sterile glass rod. Plates were incubated at room temperature under natural lighting for forty-eight hours. A stereomicroscope was used to locate the germlings. Fifty individual germinating conidia were aseptically transferred onto PDA using a sterile surgical blade (5 germlings per plate). 
Plates were incubated for seven-to-fourteen days at $20 \mathrm{C}$ with a 16:8 hour photoperiod.

Resulting colonies were scored as virulent or hypovirulent based on their cultural morphology (Figure 8).

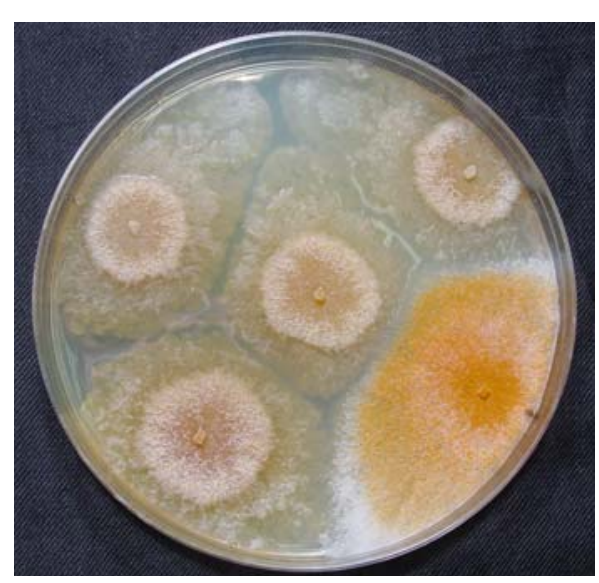

Figure 8. Single asexual spores (conidia) that have germinated on PDA. Four of the five resulting colonies are hypovirus-infected and exhibit the altered growth habit of a hypovirulent isolate.

\section{Analysis of In Vivo Asexual Sporulation by the Test Isolates}

To determine the percentage of hypovirus transmission to conidia in vivo, $7 \mathrm{~mm}$ bark disks were removed from cankers (November 2003 and July 2004) that produced stroma and returned to the laboratory. Samples were collected in 24-well tissue culture plates, and stored at -20 C. As time permitted, bark discs were removed from the freezer and soaked in warm tap water for one hour (Figure 9). Conidia were extracted from pycnidia under a stereo-microscope and then crushed in a watch-glass that contained $2 \mathrm{ml}$ of $0.1 \%$ peptone solution (Appendix B). A sterile pipette was used to transfer $1.5 \mathrm{ml}$ of the spore suspension into a $1.5 \mathrm{ml}$ microcenterfuge tube. Contents were vortexed at high speed for 45 seconds and then diluted. From each dilution, $0.5 \mathrm{ml}$ of the solution was 
pipetted onto the surface of GYE agar plates amended with antibiotics and distributed evenly using a sterile glass rod as with the in vitro study. After forty-eight hours the germlings were transferred to PDA and incubated for seven-to-fourteen days under temperature and light conditions previously described. Based on their cultural morphology, resulting colonies were scored as virulent or hypovirulent.
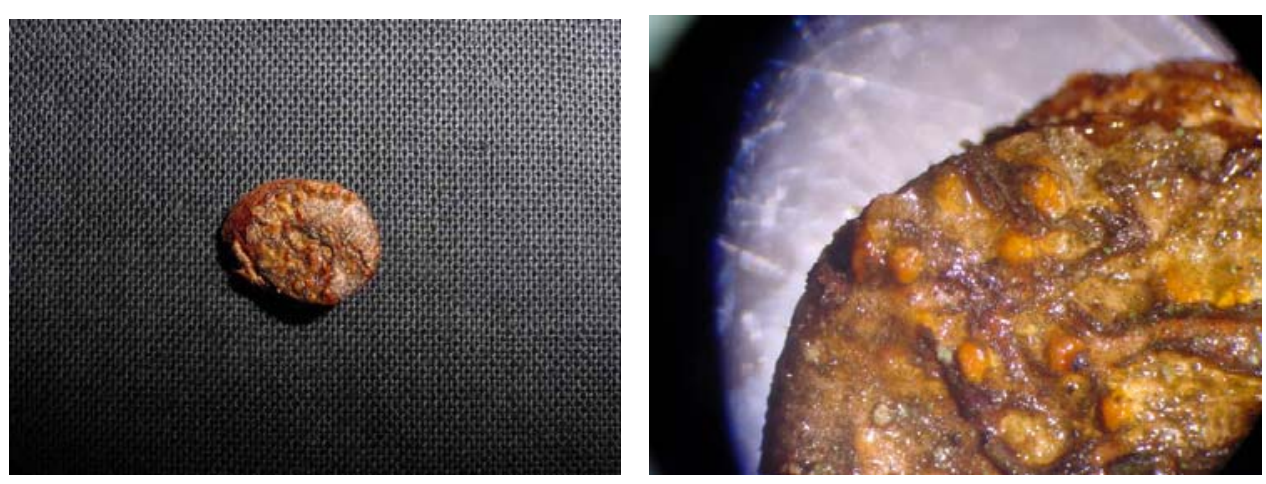

Figure 9. Seven-mm bark disc removed from canker (left). Pycnidia containing conidia on bark disc (1x) (right).

\section{Confirmation of Infecting Strain by VC Testing}

During the analysis of in vivo asexual sporulation, some stroma retrieved from the cankers established as HV yielded conidia that generated 100\% virulent progeny. Because this was a suspicious occurrence, the virulent progeny were subjected to a vegetative compatibility test to confirm their relationship to the infecting strain. To accomplish this, three pairings were made on Brom cresol green medium (Figure 10). The first pairing was the virulent single-conidial isolate in question and the test isolate used to initiate the canker. The second pairing consisted of two known compatible isolates to model a compatible reaction. The third pairing consisted of two known incompatible isolates to confirm vegetative incompatibility. Vegetative incompatibility is 
characterized by a very distinct barrage formed between the two isolates in culture. An incompatible reaction would indicate that the virulent isolate recovered was presumably the result of a wild-type infection. Conversely, a compatible reaction indicated that the isolate retrieved represented the inoculum used to create the canker.

Compatible/compatible

Unknown/Test Strain
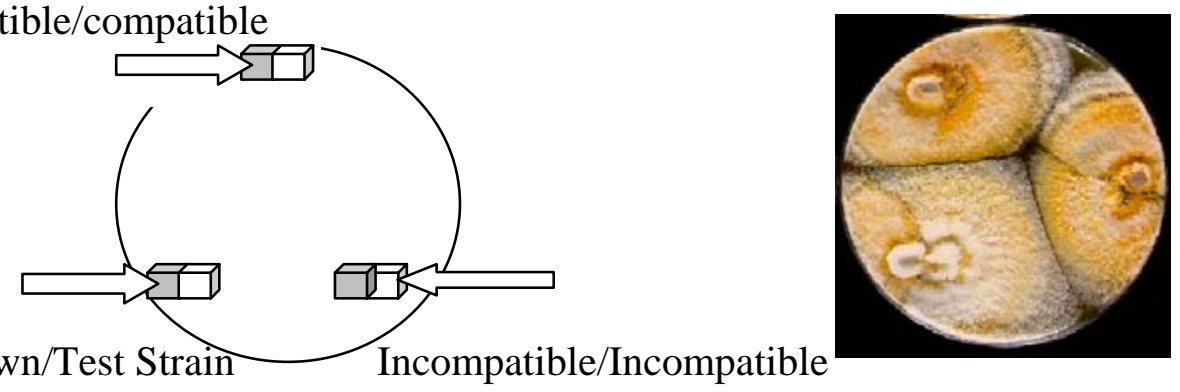

Figure 10. Schematic placement of test isolates on Brom Cresol Green medium (left). Seven-day-old cultures on Brom Cresol Green medium illustrating barrage formation (right).

\section{Hypovirus Transmission by Vegetative Incompatible Isolates via Anastomosis}

Initial VC testing was conducted to confirm that Bockenhauer, Schomberg, Euro7ss, JR10, WR2, and Ep155 were vegetatively incompatible. Generally, vegetatively incompatible isolates will form a barrage preventing anastomosis, thus, inhibiting the transfer of hypovirus dsRNA. However, hypovirus transmission has been known to occur between two vegetatively incompatible isolates. This test was conducted to determine if the infection process has an effect on vegetative incombatibility in terms of anastomosis-mediated transfer of hypovirus dsRNA.

To accomplish this, each HV test isolate was paired with each virulent, incompatible isolate. A $5 \mathrm{~mm}$ mycelium plug from a 10-to-14 day-old hypovirulent isolate was transferred to a Petri plate containing PDA. Placed adjacent to the HV mycelium plug was a 5-mm, 10-to-14 day-old virulent isolate (Figure 11). All cultures 
were incubated for three weeks at $20 \mathrm{C}$ with a 16:8 hour photoperiod then observed for conversion based on morphology. Cultures were scored as " 1 " when converted and " 0 " when conversion was not observed based on the morphological characteristics of the virulent isolate (Figure 11). Five replications of each pairing were made.

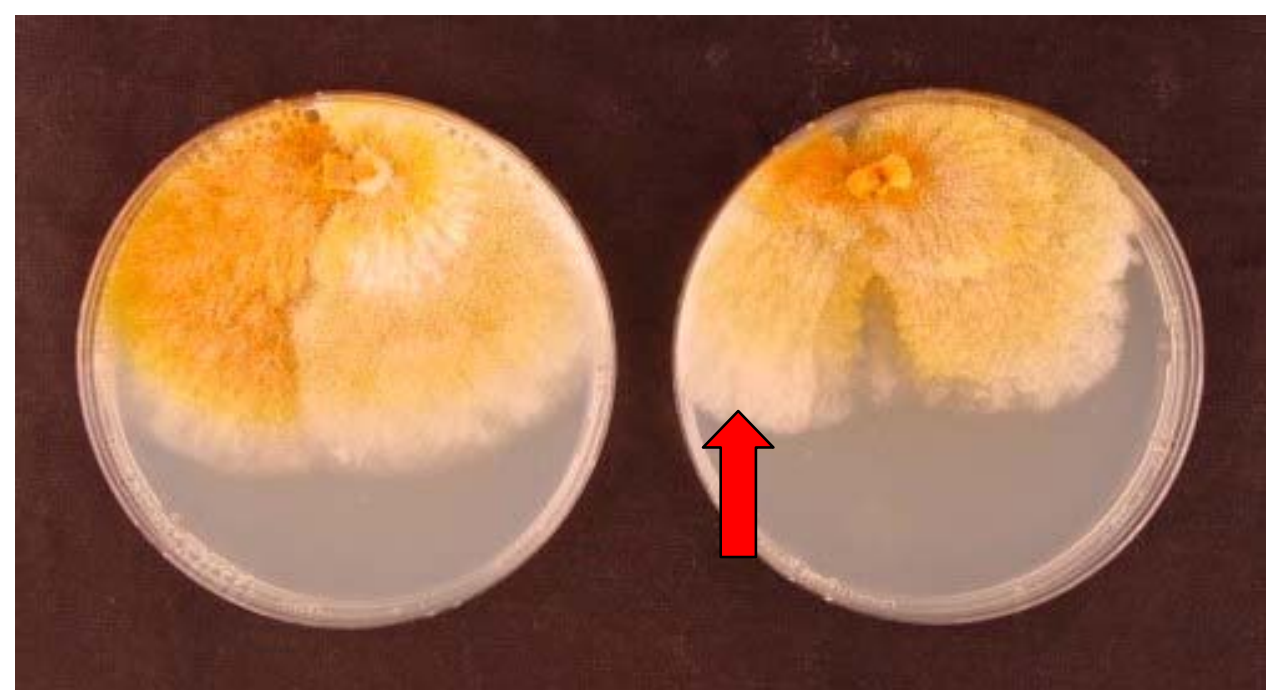

Figure 11. Barrage formation appears as a distinct line indicating horizontal transmission has been prevented (left). Red arrow denotes change in virulent morphology indicating horizontal transmission of hypovirus has occurred (right).

\section{Statistical Analysis}

A simple factorial design was used for data analysis in this study. Analysis of Variance (ANOVA) was used to detect differences in size of canker, recovery of HV isolates, and hypovirus transmission to conidia (in vitro). One-way ANOVA evaluated virulent (virus-free) isolates, CHV1-Euro7, and CHV1-Ep713 infected isolates that acquired the hypoviruses by either anastomosis or transfection by using a 1x3 factorial design. LS Means contrasts were used to further determine where significant differences 
occurred. Two-way ANOVA assessed virulent, CHV3-County Line, CHV1-Euro7, and CHV1-Ep713 isolates that acquired the hypovirus by anastomosis by using a 2x4 factorial design. Three-way ANOVA evaluated fungal isolates, method of infection, and hypoviruses using a 2x2x6 nested factorial design (virulent isolates were excluded). Oneway ANOVA with 26 treatment combinations compared isogenic anastomosed and transfected isolates using LSMeans contrasts. Tukey’s HSD test was used for multiple comparisons among the parental backgrounds. Pearson's $\chi 2$-test was used to analyze stroma production between anastomosed and transfected isolates (HV isolates were pooled and virulent isolates were excluded) and to compare hypovirus transmission to vegetative incompatible isolates. All statistics were analyzed using JMP version 5 (SAS Institute, Cary, North Carolina). These analyses were conducted with the guidance from Dr. George Seidel and Dr. James Kotcon from West Virginia University. All Analysis of Variance tables are located in Appendix F. 


\section{Results}

Of the 30 hypovirus-infected C. parasitica isolates used to initiate cankers, 26 performed like hypovirulent (HV) strains by their reduced canker growth and sporulation. Four isolates: JR10(CHV1-Ep713) anastomosed, WR2(CHV1-Ep713) transfected, Ep155(CHV1-Euro7) anastomosed and Ep155(CHV1-Euro7) transfected grew and sporulated similar to virulent isolates when they were assessed in November 2003. Double-stranded RNA extractions confirmed that these isolates did not contain hypovirus and presumably had reverted to their virulent form. Therefore, these isolates were excluded from this study.

A third set of isolates containing CHV3-County Line infected via anastomosis in each parental background was included in this field study. They were compared to the isolates containing CHV1-Euro7 and CHV1-Ep713 infected by anastomosis. Cankers produced by these isolates were contrasted to determine the influence different hypoviruses had on its fungal host in terms of size of canker, how well they retained their hypovirus, HV transmission to conidia, and HV transmission to incompatible isolates via anastomosis.

\section{Analysis of Canker Growth}

The average size $((\mathrm{L}+\mathrm{W}) / 2)$ of all virulent cankers that were initiated as controls when measured in November 2003 was 7.0 cm. Euro7ssv, Ep155, and Bockenhauer, isolates produced cankers similar in size ranging from 7.1 to $7.7 \mathrm{~cm}$ (Table 2). These isolates produced cankers significantly larger than WR2 and JR10 which averaged 6.3 and $5.9 \mathrm{~cm}$, respectively. By July 29, 2004, the average size of virulent cankers had 
increased to $18.8 \mathrm{~cm}$. When comparing the sample periods certain similarities remained consistent; virulent isolates Ep155, Bockenhauer, Schomberg, and Euro7ssv were similar in growth and significantly larger than the cankers formed by isolates JR10 and WR2.

\begin{tabular}{|c|c|c|c|c|c|}
\hline \multicolumn{3}{|c|}{ Five-Month-Old Virulent Cankers } & \multicolumn{3}{|c|}{ Twelve-Month-Old Virulent Cankers } \\
\hline t Grouping & $\begin{array}{l}\text { Parental } \\
\text { Background }\end{array}$ & Mean & t Grouping & $\begin{array}{l}\text { Parental } \\
\text { Background }\end{array}$ & Mean \\
\hline A & Euro7ssv & 7.7 & A & Bockenhauer & 20.6 \\
\hline A & Ep155 & 7.7 & A & Ep155 & 20.3 \\
\hline A & Bockenhauer & 7.6 & A & Schomberg & 19.6 \\
\hline A B & Schomberg & 7.1 & A & Euro7ssv & 19.4 \\
\hline $\mathrm{B} C$ & WR2 & 6.3 & B & JR10 & 17.8 \\
\hline $\mathrm{C}$ & JR10 & 5.9 & B & WR2 & 16.6 \\
\hline
\end{tabular}

Table 2. Tukey's HSD test contrasting size of cankers produced by the virulent isolates measured after 4 (11/12/03) and after 12 months (7/29/004). Canker sizes produced by isolates designated by the same letter are not significantly different.

The size of cankers produced by virulent isolates was significantly larger in contrast to those produced by $\mathrm{HV}$ isolates $(\mathrm{P}=0.01)$. When anastomosed and transfected isolates were compared over all treatment groups, they averaged $3.3 \mathrm{~cm}$ and $3.4 \mathrm{~cm}$, respectively (Figure 13); sizes that were not significantly different $(\mathrm{P}=0.39)$. When cankers were measured in July 2004, after twelve months of growth, HV cankers were again significantly smaller than those produced by the virulent isolates $(\mathrm{P}<0.001)$. There was little change in the size of cankers compared to the four-month measurement. They averaged $3.9 \mathrm{~cm}$ and $4.2 \mathrm{~cm}$, for the anastomosed and transfected isolates, respectively; again differences that were not significant $(\mathrm{P}=0.27)$ (Figure 13). 


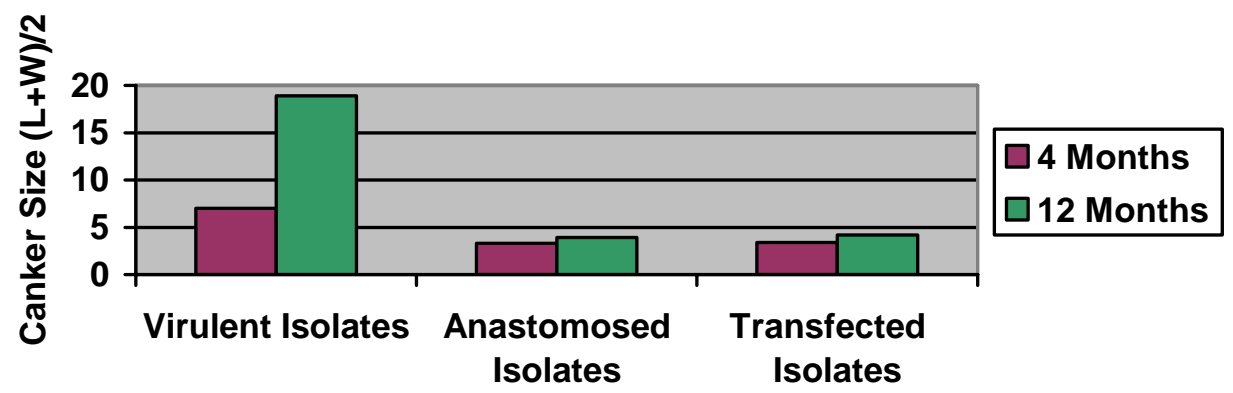

Figure 13. Average canker size produced by isolates measured after $4(11 / 12 / 03)$ and 12 months (7/29/04) when isolates were compared over all treatment groups. Larger cankers were produced by virulent isolates. Differences in the size of canker produced by anastomosed verses transfected isolates were not statistically significant.

\section{Analysis of Canker Size among Parental Backgrounds}

The factorial ANOVA indicated that a 3-way interaction occurred among isolates (parental backgrounds), hypoviruses, and method of infection when canker sizes were analyzed in November 2003 and again in July 2004 (P<0.001). Contrasts among CHV1Euro7 infected isolates measured after 4 months of growth indicated that Schomberg(CHV1-Euro7) isolates produced the largest cankers with the anastomosed isolates producing significantly larger cankers than their transfected counterparts $(\mathrm{P}=0.03) . \quad$ JR10 and WR2 anastomosed isolates produced cankers significantly smaller than those produced by all other backgrounds infected with CHV1-Euro7 (Table 3). However, the JR10 and WR2 transfected isolates produced significantly larger cankers than their anastomosed counterparts $(\mathrm{P}=0.0001)$.

When cankers were measured 12 months after initiation, the same relationships existed among anastomosed and transfected isolates for the Schomberg, JR10, and WR2 isolates. Schomberg(CHV1-Euro7) anastomosed isolates produced larger cankers than 
those produced by the transfected strains $(\mathrm{P}=0.03)$. JR10(CHV1-Euro7) and WR2(CHV1-Euro7) transfected strains produced cankers significantly larger than their anastomosed counterparts $(\mathrm{P}=0.0001)$ (Table 3$)$.

\begin{tabular}{|c|c|c|c|c|c|}
\hline \multicolumn{6}{|c|}{ Cankers Measured After 4 months: CHV1-Euro7 } \\
\hline \multicolumn{3}{|c|}{ Anastomosed } & \multicolumn{3}{|c|}{ Transfected } \\
\hline $\mathrm{t}$ & Parental Background & Mean & $\mathrm{t}$ & Parental Background & Mean \\
\hline A & Schomberg & 4.9 & A & Schomberg & 3.9 \\
\hline A $B$ & Bockenhauer & 4.1 & A B & Euro7ssv & 3.8 \\
\hline B & Euro7ssv & 3.3 & A B & Bockenhauer & 3.6 \\
\hline $\mathrm{C}$ & WR2 & 1.9 & B & WR2 & 3.1 \\
\hline $\mathrm{C}$ & JR10 & 1.8 & B & JR10 & 3.1 \\
\hline & Ep155 & NA & & Ep155 & NA \\
\hline \multicolumn{6}{|c|}{ Cankers Measured After 12 Months: CHV1-Euro7 } \\
\hline \multicolumn{3}{|c|}{ Anastomosed } & \multicolumn{3}{|c|}{ Transfected } \\
\hline $\mathrm{t}$ & Parental Background & Mean & $\mathrm{t}$ & Parental Background & Mean \\
\hline A & Schomberg & 5.9 & A & Bockenhauer & 4.9 \\
\hline A B & Bockenhauer & 4.6 & A & Schomberg & 4.8 \\
\hline B C & Euro7ssv & 4 & A & Euro7ssv & 4.7 \\
\hline C & JR10 & 2.9 & A & JR10 & 4 \\
\hline $\mathrm{C}$ & WR2 & 2.6 & A & WR2 & 3.9 \\
\hline & Ep155 & NA & & Ep155 & NA \\
\hline
\end{tabular}

Table 3. Tukey's HSD test used to contrast average sizes $((\mathrm{L}+\mathrm{W}) / 2)$ in centimeters of cankers produced by CHV1-Euro7 infected isolates recorded 4 (11/12/05) and 12 months (7/29/04) after initiation. Measurements in bold text indicate significant differences between anastomosed and transfected isolates. Isolates connected by the same letter are not significantly different.

Among CHV1-Ep713 infected isolates, Ep155 anastomosed and transfected isolates produced cankers larger than the other parental backgrounds (Table 4). The Schomberg, Euro7ssv, JR10, and WR2 isolates produced cankers similar in size. The only statistical difference observed when comparing anastomosed and transfected isolates was with the Bockenhauer isolates; the transfected isolates produced significantly smaller cankers than their anastomosed counterparts $(\mathrm{P}=0.0002)$ (Table 4). 
After 12 months of growth, canker sizes remained similar to those when measured at 4 months. As in the November 2003 measurements, Ep155 isolates produced significantly larger cankers when compared to all but the Bockenhauer(CHV1-Ep713) anastomosed cankers, which remained significantly larger than its transfected counterpart $(\mathrm{P}=0.0010)$.

\begin{tabular}{|c|c|c|c|c|c|}
\hline \multicolumn{6}{|c|}{ Cankers Measured After 4 months: CHV1-Ep713 } \\
\hline \multicolumn{3}{|c|}{ Anastomosed } & \multicolumn{3}{|c|}{ Transfected } \\
\hline $\mathrm{t}$ & Parental Background & Mean & $\mathrm{t}$ & Parental Background & Mean \\
\hline A & Ep155 & 4.7 & A & Ep155 & 4.8 \\
\hline A & Bockenhauer & 4.5 & $\mathrm{~B}$ & JR10 & 3.5 \\
\hline $\mathrm{B}$ & Euro7ssv & 2.6 & B & Bockenhauer & 2.9 \\
\hline $\mathrm{B}$ & Schomberg & 2.6 & $\mathrm{~B}$ & Euro7ssv & 2.9 \\
\hline \multirow[t]{2}{*}{ B } & WR2 & 2.5 & $\mathrm{~B}$ & Schomberg & 2.8 \\
\hline & JR10 & NA & & WR2 & NA \\
\hline \multicolumn{6}{|c|}{ Cankers Measured After 12 Months: CHV1-Ep713 } \\
\hline \multicolumn{3}{|c|}{ Anastomosed } & \multicolumn{3}{|c|}{ Transfected } \\
\hline $\mathrm{t}$ & Parental Background & Mean & $\mathrm{t}$ & Parental Background & Mean \\
\hline A & Ep155 & 5.3 & A & Ep155 & 5.5 \\
\hline A & Bockenhauer & 4.8 & A B & JR10 & 4.5 \\
\hline B & WR2 & 3.2 & B & Schomberg & 3.5 \\
\hline $\mathrm{B}$ & Euro7ssv & 3.1 & B & Euro7ssv & 3.5 \\
\hline B & Schomberg & 2.9 & B & Bockenhauer & 3.4 \\
\hline & JR10 & NA & & WR2 & NA \\
\hline
\end{tabular}

Table 4. Tukey's HSD test used to contrast average sizes $((\mathrm{L}+\mathrm{W}) / 2)$ in centimeters of cankers produced by CHV1-Ep713 infected isolates recorded 4 (11/12/03) and 12 months (7/29/04) after initiation. Measurements in bold text indicate significant differences between anastomosed and transfected isolates. Isolates connected by the same letter are not significantly different.

\section{Canker Sizes among CHV3-County Line, CHV1-Euro7, and CHV1-Ep713 Isolates}

Isolates infected with CHV3-County Line were added to the comparison of isolates that acquired hypoviruses via anastomosis. CHV3-County Line-infected isolates, regardless of parental background, produced significantly smaller cankers than isolates 
containing either the CHV1-type hypovirus (CHV1-Euro7 or CHV1-Ep713) when measured after 5 and 12 months (Figure 14).

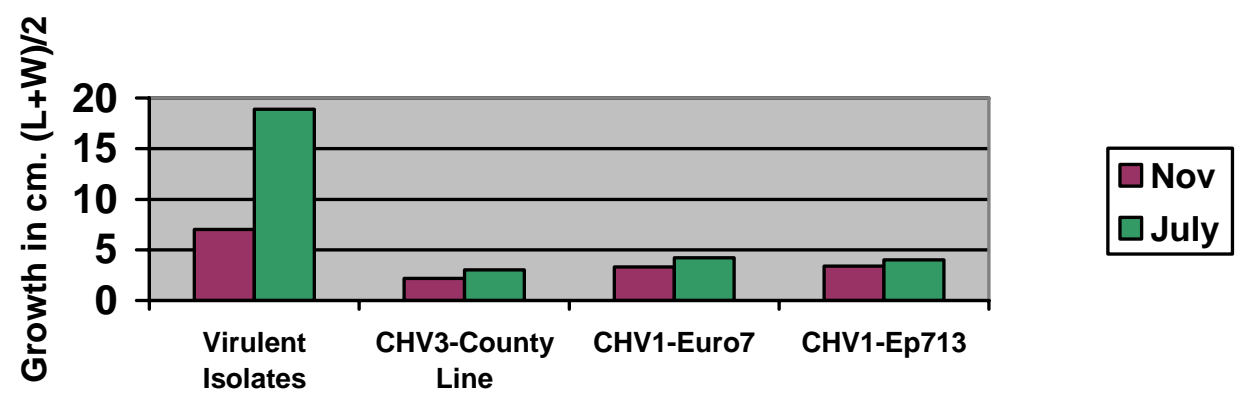

Figure 14. Average canker size produced by all anastomosed isolates when cankers were measured after 4 (11/12/03) and 12 months (7/29/04). Cankers produced by CHV3-County Line-infected isolates were significantly smaller. CHV1-Euro7 and CHV1-Ep713 data are repeated from Figure 13.

Significant 2-way interactions occurred among parental backgrounds and hypoviruses $(\mathrm{P}<0.001)$. When comparing canker sizes among the fungal genomes, CHV3-County Line infected isolates produced cankers similar in size regardless of the fungal background (Table 5). The Bockenhauer, Schomberg, and Ep155 isolates infected with CHV3-County Line produced significantly smaller cankers when compared to their isogenic counterparts infected with either CHV1-type hypovirus. Euro7ssv, JR10, and WR2 isolates produced cankers similar in size regardless of the hypovirus they contained. Isolates infected with CHV1-Euro7 or CHV1-Ep713 displayed variations in canker sizes dependent on the parental background; a trait that was not observed when isolates were infected by CHV3-County Line (Table 5). 


\begin{tabular}{|c|c|c|c|c|c|c|c|c|}
\hline \multicolumn{9}{|c|}{ Cankers Measured 5 Months After Initiation } \\
\hline \multicolumn{3}{|c|}{ CHV3-County Line } & \multicolumn{3}{|c|}{ CHV1-Euro7 } & \multicolumn{3}{|c|}{ CHV1-Ep713 } \\
\hline $\mathbf{t}$ & Background & $\mathbf{x}$ & $\mathbf{t}$ & Background & $\mathbf{x}$ & $\mathbf{t}$ & Background & $\mathbf{x}$ \\
\hline A & Euro7ssv & 2.6 & A & Schomberg & 4.9 & A & Ep155 & 4.7 \\
\hline A & JR10 & 2.3 & $\mathrm{AB}$ & Bockenhauer & 4.1 & A & Bockenhauer & 4.5 \\
\hline A & Schomberg & 2.3 & $\mathrm{~B}$ & Euro7ssv & 3.3 & $\mathrm{~B}$ & Euro7ssv & 2.6 \\
\hline A & Bockenhauer & 2.1 & $\mathrm{C}$ & WR2 & 1.9 & B & Schomberg & 2.6 \\
\hline A & Ep155 & 2.1 & $\mathrm{C}$ & JR10 & 1.8 & B & WR2 & 2.5 \\
\hline A & WR2 & 1.9 & & Ep155 & NA & & JR10 & NA \\
\hline \multicolumn{9}{|c|}{ Cankers Measured 12 Months After Initiation } \\
\hline \multicolumn{3}{|c|}{ CHV3-County Line } & \multicolumn{3}{|c|}{ CHV1-Euro7 } & \multicolumn{3}{|c|}{ CHV1-Ep713 } \\
\hline $\mathbf{t}$ & Background & $\mathbf{x}$ & $\mathbf{t}$ & Background & $\mathbf{x}$ & $\mathbf{t}$ & Background & $\mathbf{x}$ \\
\hline A & Schomberg & 3.6 & A & Schomberg & 5.9 & A & Ep155 & 5.3 \\
\hline A & Euro7ssv & 3.3 & $\mathrm{AB}$ & Bockenhauer & 4.6 & A & Bockenhauer & 4.8 \\
\hline A & JR10 & 3.3 & $\mathrm{BC}$ & Euro7ssv & 4 & B & WR2 & 3.2 \\
\hline A & Ep155 & 3.3 & $\mathrm{C}$ & JR10 & 2.9 & B & Euro7ssv & 3.1 \\
\hline A & WR2 & 2.7 & $\mathrm{C}$ & WR2 & 2.6 & B & Schomberg & 2.9 \\
\hline A & Bockenhauer & 2.7 & & Ep155 & NA & & JR10 & NA \\
\hline
\end{tabular}

Table 5. Tukey's HSD test used to contrast average sizes $((\mathrm{L}+\mathrm{W}) / 2)$ in centimeters of cankers produced by CHV3-County Line, CHV1-Euro7, and CHV1-Ep713 infected isolates recorded $4(11 / 12 / 03)$ and 12 months (7/29/04) after initiation. CHV1-Euro7 and CHV1-Ep713 data are repeated from Tables 3 and 4 . Measurements in bold text indicate significant differences among hypoviruses. Isolates connected by the same letter are not significantly different. 


\section{Bark Sample Analysis to Evaluate Hypovirus Persistence}

The morphology of the isolates cultured from bark plugs removed from cankers formed the basis for virulence or hypovirulence determination. Fungi isolated from seventy-eight percent of the individual bark plugs extracted from the 4-month-old control (virulent) cankers were scored as virulent isolates that were used to initiate the cankers (Figure 15). The balance (22\%) of the isolates recovered were categorized as other fungi isolated or bark plugs that produced no colonies. When the control cankers were sampled in July 2004, 91\% of the bark samples produced parental virulent colonies and 9\% represented other fungi or were sterile (Figure 15). No hypovirulent isolates were retrieved from the virulent control cankers at either date.

In contrast to the isolations from virulent cankers, frequency of recovery of virusinfected C. parasitica from cankers initiated by HV isolates was lower and the percentage of non-C. parasitica isolates recovered was dramatically higher (Figure 15). When comparisons were made between anastomosed and transfected isolates, regardless of hypovirus or parental background, there were no differences in the percentage of $\mathrm{HV}$ isolates retrieved when sampled in November $2003(\mathrm{P}=0.59)$. Isolation results for the anastomosed isolates were: $29 \% \mathrm{HV}, 18 \% \mathrm{~V}$, and 53\% non-C parasitica (Figure 15). The transfected isolates yielded 32\% HV, 10\% V, and 58\% non-C parasitica (Figure 15).

As noted with isolations from the virulent cankers, recovery of $\mathrm{HV}$ isolates increased from November 2003 to July 2004 (Figure 15). Bark plugs cultured from the July, 2004 sample yielded 38\% HV, 21\% V, and 41\% non-C. parasitica recovery from samples removed from anastomosed cankers and $47 \% \mathrm{HV}, 18 \% \mathrm{HV}$, and $35 \%$ non-C. parasitica were from the transfected group (Figure 15). Although HV recovery was 
higher among the transfected isolates, this difference was not statistically significant $(\mathrm{P}=0.07)$.

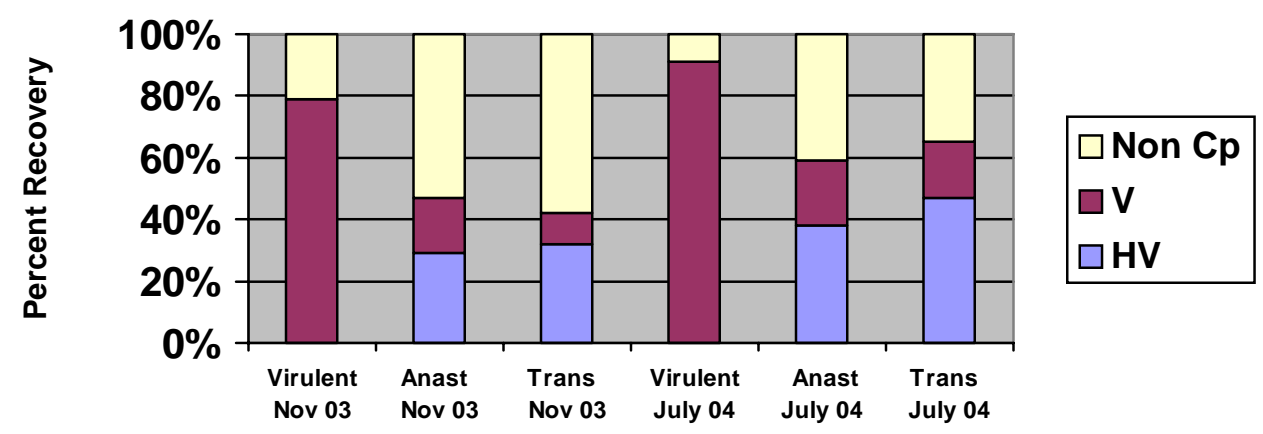

Figure 15. Comparison of the percentage of parental hypovirulent (HV), virulent (V), and non-C. parastica (Non Cp) isolates without regard to hypovirus or fungal host. Bark plugs were recovered from cankers after 4 (11/12/03) and 12 months (7/29/04). No significant differences existed between anastomosed and transfected isolates when isolates were compared over all treatment groups.

\section{Bark Sample Analysis among Parental Backgrounds}

Among the CHV1-Euro7 infected isolates sampled in November 2003, there were no differences in the percentages of $\mathrm{HV}$ isolates recovered between anastomosed and transfected isolates. Bockenhauer and Schomberg yielded the highest recovery percentages of $\mathrm{HV}$ isolates from cankers when compared to Euro7ssv, JR10, and WR2 isolates (Table 6). In the July 2004 sampling, a 3-way interaction occurred among parental backgrounds, hypoviruses, and method of infection $(\mathrm{P}=0.01)$. The statistical difference between anastomosed and transfected isolates was detected with the WR2 background. Cankers initiated by this isolate when transfected yielded a higher percentage of $\mathrm{HV}$ when compared to the cankers produced by the anastomosed isolates; $50 \%$ to $21 \%$, respectively ( $\mathrm{P}=0.04$ ) (Table 6 ). The percentage of $\mathrm{HV}$ isolated increased in July 2004 within the CHV1-Euro7 anastomosed and transfected group. All rankings 
and statistical similarities remained the same between the two sample periods when parental backgrounds were contrasted (Table 6).

\begin{tabular}{|c|c|c|c|c|c|}
\hline \multicolumn{6}{|c|}{$\begin{array}{l}\text { Percent HV Isolates Recovered from Cankers Sampled After } 4 \text { months: } \\
\text { CHV1-Euro7 }\end{array}$} \\
\hline \multicolumn{3}{|c|}{ Anastomosed } & \multicolumn{3}{|c|}{ Transfected } \\
\hline $\mathrm{t}$ & Parental Background & Mean & $\mathrm{t}$ & Parental Background & Mean \\
\hline A & Bockenhauer & $71 \%$ & $\mathrm{~A}$ & Bockenhauer & $48 \%$ \\
\hline A & Schomberg & $46 \%$ & A B & Schomberg & $38 \%$ \\
\hline B & Euro7ssv & $8 \%$ & A B & Euro7ssv & $33 \%$ \\
\hline B & JR10 & $8 \%$ & A B & WR2 & $25 \%$ \\
\hline \multirow[t]{2}{*}{ B } & WR & $8 \%$ & B & JR10 & $17 \%$ \\
\hline & Ep155 & NA & & Ep155 & NA \\
\hline \multicolumn{6}{|c|}{$\begin{array}{l}\text { Percent HV Isolates Recovered from Cankers Sampled After } 12 \text { months: } \\
\text { CHV1-Euro7 }\end{array}$} \\
\hline \multicolumn{3}{|c|}{ Anastomosed } & \multicolumn{3}{|c|}{ Transfected } \\
\hline $\mathrm{t}$ & Parental Background & Mean & $\mathrm{t}$ & Parental Background & Mean \\
\hline A & Bockenhauer & $71 \%$ & $\mathrm{~A}$ & Bockenhauer & $63 \%$ \\
\hline $\mathrm{B}$ & Schomberg & $38 \%$ & A B & Schomberg & $50 \%$ \\
\hline B C & WR2 & $21 \%$ & A B & WR2 & $50 \%$ \\
\hline B C & Euro7ssv & $17 \%$ & A B & Euro7ssv & $44 \%$ \\
\hline \multirow[t]{2}{*}{$\mathrm{C}$} & JR10 & $13 \%$ & B & JR10 & $33 \%$ \\
\hline & Ep155 & NA & & Ep155 & NA \\
\hline
\end{tabular}

Table 6. Tukey's HSD test contrasting \% HV recovered from cankers produced by CHV1-Euro7 infected isolates sampled after 4 (11/12/03) and 12 months (7/29/04). Bold text indicates significant differences between anastomosed and transfected isolates. Means designated by the same letter are not significantly different. These data are presented visually in histograms in Appendix E.

There were no differences in the percentages of $\mathrm{HV}$ isolates recovered between anastomosed and transfected isolates infected with CHV1-Ep713 when cankers were sampled in November 2003. Among the fungal genomes, HV isolates were recovered from Ep155(CHV1-Ep713) initiated cankers at a significantly higher rates (Table 7). Among the anastomosed isolates, WR2 and Euro7ssv had a significantly lower 
percentage of $\mathrm{HV}$ isolates recovered from bark plugs when sampled after 4 months (Table 7). Among the transfected group, JR10, Bockenhauer, Schomberg, and Euro7ssv were all similar at both time periods (Table 7).

In samples collected in July 2004, there was an increase in the percentage of HV isolates recovered and a decrease in non-C. parasitica from cankers initiated by all isolates except Ep155. Schomberg, Bockenhauer, and Ep155 did not differ statistically in percentage of $\mathrm{HV}$ isolated from cankers among the anastomosed isolates and all were greater than WR2 or Euro7ssv. This trend was reversed among the transfected isolates; these backgrounds were similar and had the lowest percentages of $\mathrm{HV}$ isolates recovered from cankers (Table 7). Two significant differences were detected between CHV1Ep713 anastomosed and transfected isolates during this sample period. Isolations from Euro7ssv transfected isolates (46\%) were significantly higher than their anastomosed counterparts (8\%) $(\mathrm{P}=0.02)$. Conversely, Schomberg anastomosed cankers yielded a higher percentage of $\mathrm{HV}$ isolates than those recovered from cankers initiated by the transfected isolates; $71 \%$ verses $42 \%$, respectively $(\mathrm{P}=0.04)$. 


\begin{tabular}{|c|c|c|c|c|c|}
\hline \multicolumn{6}{|c|}{$\begin{array}{l}\text { Percent HV Isolates Recovered from Cankers Sampled After } 4 \text { months: } \\
\text { CHV1-Ep713 }\end{array}$} \\
\hline \multicolumn{3}{|c|}{ Anastomosed } & \multicolumn{3}{|c|}{\begin{tabular}{|c|} 
Transfected \\
\end{tabular}} \\
\hline $\mathrm{t}$ & Parental Background & Mean & $\mathrm{t}$ & Parental Background & Mean \\
\hline A & Ep155 & $67 \%$ & A & Ep155 & $58 \%$ \\
\hline B & Bockenhauer & $38 \%$ & A B & JR10 & $33 \%$ \\
\hline $\mathrm{BC}$ & Schomberg & $33 \%$ & A B & Schomberg & $31 \%$ \\
\hline C D & WR2 & $10 \%$ & B & Euro7ssv & $21 \%$ \\
\hline \multirow[t]{2}{*}{$\mathrm{D}$} & Euro7ssv & $4 \%$ & B & Bockenhauer & $17 \%$ \\
\hline & JR10 & NA & & WR2 & NA \\
\hline \multicolumn{6}{|c|}{$\begin{array}{l}\text { Percent HV Isolates Recovered from Cankers Sampled After } 12 \text { months: } \\
\text { CHV1-Ep713 }\end{array}$} \\
\hline \multicolumn{3}{|c|}{ Anastomosed } & \multicolumn{3}{|c|}{ Transfected } \\
\hline $\mathrm{t}$ & Parental Background & Mean & $\mathrm{t}$ & Parental Background & Mean \\
\hline A & Schomberg & $71 \%$ & A & JR10 & $62 \%$ \\
\hline A & Bockenhauer & $67 \%$ & A & Euro7ssv & $46 \%$ \\
\hline A & Ep155 & $63 \%$ & A & Bockenhauer & $42 \%$ \\
\hline $\mathrm{B}$ & WR2 & $10 \%$ & A & Ep155 & $42 \%$ \\
\hline \multirow[t]{2}{*}{ B } & Euro7ssv & $8 \%$ & A & Schomberg & $42 \%$ \\
\hline & JR10 & NA & & WR2 & NA \\
\hline
\end{tabular}

Table 7. Tukey's HSD test contrasting \% HV recovered from cankers produced by CHV1-Ep713 infected isolates sampled after 4 (11/12/03) and 12 months (7/29/04). Bold text indicates significant differences between anastomosed and transfected isolates. Means designated by the same letter are not significantly different. These data are presented visually in histograms in Appendix E.

Bark Sample Analysis among Anastomosed CHV3-County Line, CHV1-Euro7, and

\section{CHV1-Ep713 Isolates}

In November 2003, CHV3-County Line (14\%) isolates infected via anastomosis

were recovered at significantly lower rates than the anastomosed CHV1-Euro7 (29\%) and CHV1-Ep713 (31\%) ( $\mathrm{P}=0.03$ and 0.01). However after 12 months, HV recovery was statistically similar among the three hypoviruses (Figure 16). 


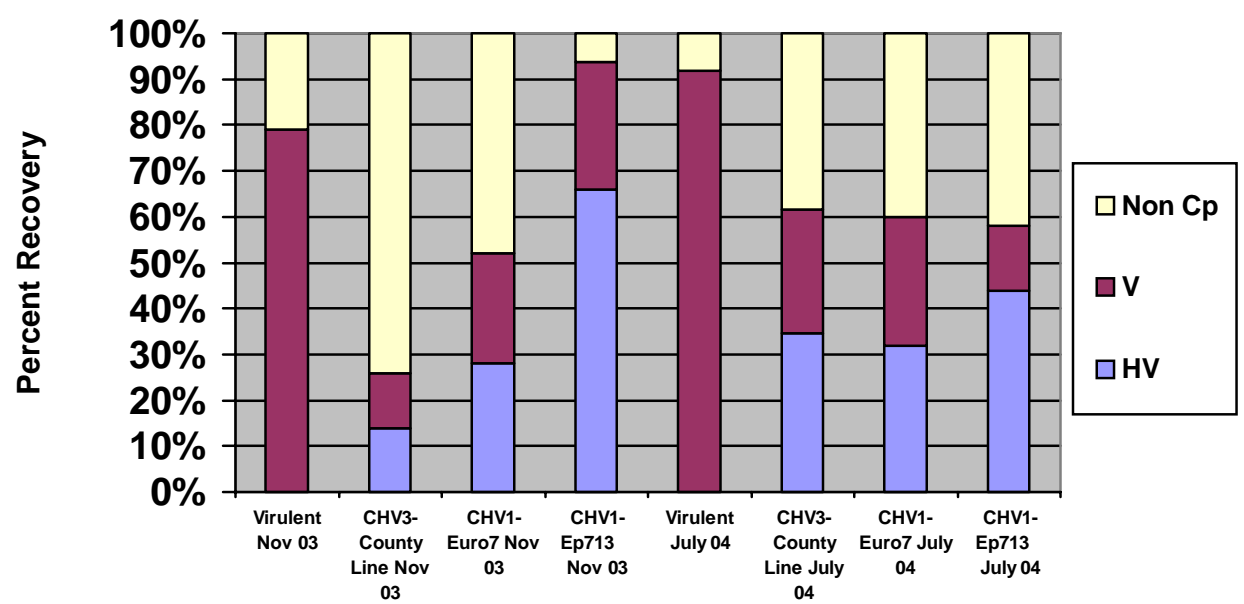

Figure 16. Comparison of CHV3- County Line, CHV1-Euro7, and CHV1-Ep713 hypoviruses in terms of percentage of hypovirulent (HV), virulent (V), and non-C. parastica (Non Cp) isolates recovered from all cankers initiated by anastomosed isolates and sampled after 4 (11/12/03) and 12 months (7/29/04). CHV1-Euro7 and CHV1-Ep713 data are repeated from Figure 15.

After four months there were significant 2-way interactions among the parental backgrounds and the three hypoviruses $(\mathrm{P}=0.001)$. County Line-infected Bockenhauer, Schomberg, and Ep155 were recovered at significantly lower percentages than their CHV1-type counterparts. In contrast, Euro7ssv, JR10, and WR2 backgrounds generated similar HV percentages, regardless of the infecting hypovirus (Table 8).

Significant 2-way interactions were detected 12 months after canker initiation $(\mathrm{P}=0.0001)$. CHV3-County Line-infected isolates again were recovered at lower rates in cankers from Bockenhauer, Schomberg, and Ep155 isolates (Table 8). Conversely, percent HV generated from Euro7ssv(CHV3-County Line) and JR10(CHV3-County Line) were recovered at significantly higher rates than the isolates infected with CHV1type hypoviruses (Table 8). Recovery of HV isolates in WR2 was similar regardless of the infecting hypovirus. 


\begin{tabular}{|c|c|c|c|c|c|c|c|c|}
\hline \multicolumn{9}{|c|}{ Cankers Measured 5 Months After Initiation } \\
\hline \multicolumn{3}{|c|}{ CHV3-County Line } & \multicolumn{3}{|c|}{ CHV1-Euro7 } & \multicolumn{3}{|c|}{ CHV1-Ep713 } \\
\hline $\mathbf{t}$ & Background & $\%$ & $\mathbf{t}$ & Background & $\%$ & $\mathbf{t}$ & Background & $\%$ \\
\hline A & Euro7ssv & 25 & A & Bockenhauer & 71 & A & Ep155 & 67 \\
\hline A & WR2 & 25 & A & Schomberg & 46 & B & Bockenhauer & 38 \\
\hline A & Bockenhauer & 19 & B & Euro7ssv & 8 & $\mathrm{BC}$ & Schomberg & 33 \\
\hline A & Schomberg & 17 & $\mathrm{~B}$ & JR10 & 8 & CD & WR2 & 10 \\
\hline A & Ep155 & 13 & B & WR2 & 8 & $\mathrm{D}$ & Euro7ssv & 4 \\
\hline A & JR10 & 8 & & Ep155 & NA & & JR10 & NA \\
\hline \multicolumn{9}{|c|}{ Cankers Measured 12 Months After Initiation } \\
\hline \multicolumn{3}{|c|}{ CHV3-County Line } & \multicolumn{3}{|c|}{ CHV1-Euro7 } & \multicolumn{3}{|c|}{ CHV1-Ep713 } \\
\hline $\mathbf{t}$ & Background & $\%$ & $\mathbf{t}$ & Background & $\%$ & $\mathbf{t}$ & Background & $\%$ \\
\hline $\mathrm{A}$ & Euro7ssv & 52 & $\mathrm{~A}$ & Bockenhauer & 71 & A & Schomberg & 71 \\
\hline A & Bockenhauer & 43 & $\mathrm{~B}$ & Schomberg & 38 & A & Bockenhauer & 67 \\
\hline A & JR10 & 42 & B C & WR2 & 21 & A & Ep155 & 63 \\
\hline A & WR2 & 29 & B C & Euro7ssv & 17 & $\mathrm{~B}$ & WR2 & 10 \\
\hline A & Schomberg & 28 & $\mathrm{C}$ & JR10 & 13 & B & Euro7ssv & 8 \\
\hline A & Ep155 & 13 & & Ep155 & NA & & JR10 & NA \\
\hline
\end{tabular}

Table 8. Tukey's HSD test contrasting \% HV recovered from cankers produced by anastomosed CHV3-County Line-infected isolates sampled after 4 (11/12/03) and 12 months (7/29/04). CHV1-Euro7 and CHV1-Ep713 data are repeated from Tables 6 and 7. Percentages in bold text indicate significant differences among hypoviruses. Means designated by the same letter are not significantly different. These data are presented visually in histograms in Appendix E.

\section{Canker Stroma Rating}

All cankers were scored on a subjective scale of $0-3$ ( $3=$ abundant stromata) for the production of pycnidia. Virulent cankers used as controls collectively had an average stroma rating of 2.78 when evaluated in November 2003. By July 2004, stromata production had increased to a rating of 3.0 for cankers produced by virulent isolates. The majority of the hypovirulent (HV) C. parasitica isolates used to initiate cankers did not produce stroma. In November 2003, the average stroma rating on cankers produced by all anastomosed and transfected isolates, regardless of hypovirus or parental background, was 0.30 and 0.41 , respectively (Figure 17). These differences were not significant $(\mathrm{P}=$ 
0.59). When assessed in July, stroma ratings decreased to 0.04 for anastomosed and 0.02 for transfected isolates (Figure 17). Again, these differences were not statistically significant $(\mathrm{P}=0.44)$.

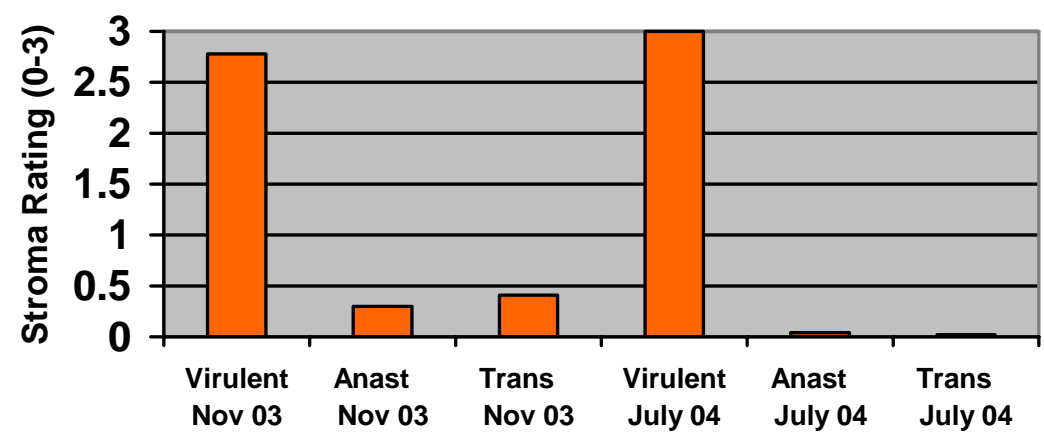

Figure 17. Comparison of the stroma rating (0-3) produced by cankers initiated by Euro7 and Ep713-infected isolates evaluated after 4 (11/12/03) and 12 (7/29/04). There was no statistical difference between anastomosed and transfected isolates when isolates were compared over all treatment groups.

\section{Comparison of HV Transmission to Conidia in vivo}

Single-conidia harvested from pycnidia of HV initiated cankers are reported as the percent of conidia to which hypovirus was transmitted based on the number of germlings that produced colonies with hypovirulent morphology. Data are presented only for cankers that produced stromata (Tables $9,10,11$ ). Due to the limited number of stroma produced by hypovirus-infected isolates, a statistical analysis was not performed.

In November 2003 only 31 of $192 \mathrm{HV}$ isolates formed cankers that produced stroma. Furthermore, of the 31 cankers, only 15 produced stroma from which conidia could be obtained from the bark discs. Six of these represented stroma from cankers initiated by anastomosed isolates and nine were from cankers initiated by transfected 
isolates. Of the six samples taken from cankers initiated by anastomosed isolates, the $\mathrm{HV}$ transmission to conidia averaged $44 \%$ (10\% - 94\%) compared to $25 \%$ (4\% - 70\%) HV transmission to conidia by the transfected isolates (Figure 18).

In July 2004, only 8 HV cankers produced visible stroma. Of these, stroma containing viable conidia were recovered from only 5 cankers; 3 cankers initiated by anastomosed isolates and 2 cankers produced by transfected isolates. The average percent of hypovirus transmission to conidia from stroma sampled from cankers initiated by anastomosed and transfected isolates was similar; 31\% (6\%-48\%) and 26\% (4\% and 48\%), respectively (Figure 18).

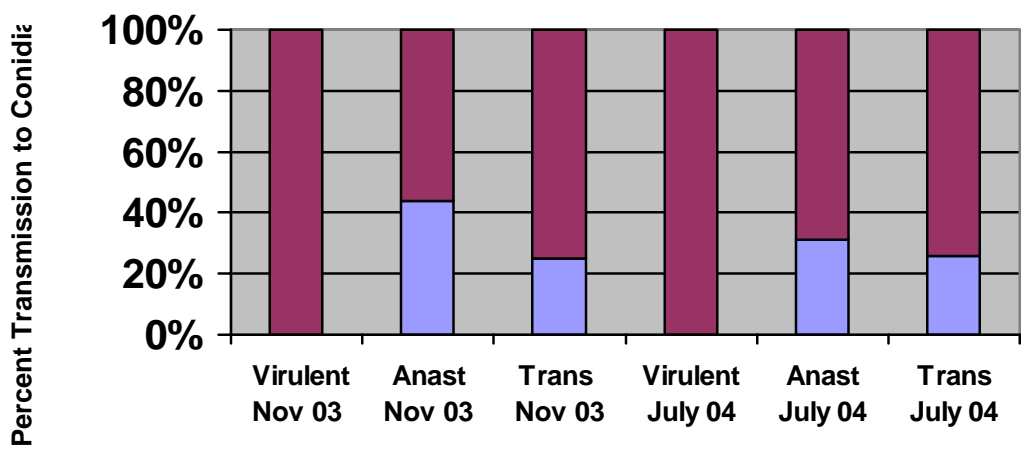

Figure 18. The percentage of hypovirulent (HV) and virulent (V) colonies that resulted from pycnidia produced by anastomosed and transfected isolates, regardless of hypovirus or parental background, sampled after 4 (11/12/03) and 12 months (7/29/04). 


\section{Asexual Reproduction and HV Transmission after 5 and 12 Months: CHV1-Euro7}

Stroma production was inconsistent and in some cases only a few of the replicates produced stroma in vivo. Of the CHV1-Euro7-infected isolates, Schomberg had the highest production of stroma as well as the highest percentage of $\mathrm{HV}$ transmission to conidia when sampled November 2003. When anastomosed and transfected isolates were compared, the anastomosed Schomberg(CHV1-Euro7) isolates produced more stroma and had a higher percentage of HV transmission than the Schomberg transfected isolates (Table 9). In contrast, cankers initiated by transfected Bockenhauer(CHV1-Euro7) and Euro7ssv(CHV1-Euro7) isolates generated a greater amount of stroma and a higher percentage of HV transmission than their anastomosed counterparts (Table 9). JR10 and WR2 did not produce stroma, thus HV transmission rates could not be determined.

Fewer stroma were evident in July for cankers produced by HV isolates. After 12-months, the anastomosed Schomberg isolate remained one of the better stroma producers of HV conidia (Table 9). The Bockenhauer anastomosed and transfected isolates produced limited stroma and when spores were isolated, relatively few had the morphology typical of HV isolates. WR2, which did not generate stroma in November, produced one canker initiated by a transfected isolate that yielded $48 \% \mathrm{HV}$ conidia in July (Table 9). JR10 and Euro7ssv isolates did not produce stroma after 12 months. 


\begin{tabular}{|c|c|c|c|c|c|c|}
\hline \multicolumn{7}{|c|}{ Ratings of Asexual Reproduction After 4 months: CHV1-Euro7 } \\
\hline Anastomosed & Bockenhauer & Schomberg & Euro7ssv & JR10 & WR2 & Ep155 \\
\hline Stroma Rating & 0.38 & 1.71 & 0.13 & 0 & 0 & NA \\
\hline \# of cankers & 2 of 8 & 6 of 8 & 1 of 8 & 0 & 0 & \\
\hline Viable stroma & 0 of 2 & 4 of 6 & 0 of 1 & 0 & 0 & \\
\hline$\%$ HV conidia & - & $\mathbf{6 0 \%}$ & - & - & - & \\
\hline Transfected & Bockenhauer & Schomberg & Euro7ssv & JR10 & WR2 & Ep155 \\
\hline Stroma Rating & 1.33 & 1.33 & 1.0 & 0 & 0 & NA \\
\hline \# of cankers & 5 of 8 & 4 of 8 & 4 of 8 & 0 & 0 & \\
\hline Viable stroma & 3 of 5 & 0 of 4 & 4 of 4 & 0 & 0 & \\
\hline$\%$ HV conidia & $\mathbf{2 7} \%$ & - & $\mathbf{2 8 \%}$ & - & - & \\
\hline Ratings of Asexual Reproduction After 12 Months: CHV1-Euro7 \\
\hline Anastomosed & Bockenhauer & Schomberg & Euro7ssv & JR10 & WR2 & Ep155 \\
\hline Stroma Rating & 0.06 & 0.21 & 0 & 0 & 0 & NA \\
\hline \# of cankers & 1 of 8 & 1 of 7 & 0 & 0 & 0 & \\
\hline Viable stroma & 1 of 1 & 1 of 1 & 0 & 0 & 0 & \\
\hline \% HV & $\mathbf{6} \%$ & $\mathbf{3 8 \%}$ & - & - & - & \\
\hline Transfected & Bockenhauer & Schomberg & Euro7ssv & JR10 & WR2 & Ep155 \\
\hline Stroma Rating & 0.07 & 0.07 & 0 & 0 & 0.08 & NA \\
\hline \# of cankers & 1 of 8 & 1 of 7 & 0 & 0 & 1 of 5 & \\
\hline Viable stroma & 1 of 7 & 0 of 1 & 0 & 0 & 1 of 1 & \\
\hline \% HV & $\mathbf{4} \%$ & - & - & - & $\mathbf{4 8 \%}$ & \\
\hline
\end{tabular}

Table 9. Average stroma rating and percentage of HV transmission to conidia from CHV1Euro7 isolates rated and sampled after 4 (11/12/03) and 12 months (7/29/04).

\section{Asexual Repoduction and HV Transmission after 5 and 12 Months: CHV1-Ep713}

When infected with CHV1-Ep713, significantly fewer stroma were produced than when CHV1-Euro7 was the infecting hypovirus $(\mathrm{P}=0.001)$. Bockenhauer and Ep155 isolates were the only isolates to produce stroma when cankers were rated and sampled in November 2003 (Table 10). Although the Bockenhauer isolates produced stroma, no conidia were recovered. The anastomosed and transfected Ep155 isolates produced similar percentages of HV inoculum (Table 10). In July 2004, the only CHV1-Ep713 cankers that produced stroma were those cankers initiated by the anastomosed Bockenhauer isolates (Table 10). 


\begin{tabular}{|c|c|c|c|c|c|c|}
\hline \multicolumn{7}{|c|}{ Ratings of Asexual Reproduction After 4 months: CHV1-Ep713 } \\
\hline Anastomosed & Bockenhauer & Schomberg & Euro7ssv & JR10 & WR2 & Ep155 \\
\hline Stroma Rating & 0.30 & 0 & 0 & 0 & 0 & 0.70 \\
\hline \# of cankers & 1 of 5 & 0 & 0 & 0 & 0 & 3 of 5 \\
\hline Viable stroma & 0 & 0 & 0 & 0 & 0 & 2 of 3 \\
\hline \% HV conidia & - & - & - & - & - & $12 \%$ \\
\hline Transfected & Bockenhauer & Schomberg & Euro7ssv & JR10 & WR2 & Ep155 \\
\hline Stroma Rating & 0.14 & 0 & 0 & 0 & 0 & 0.92 \\
\hline \# of cankers & 1 of 7 & 0 & 0 & 0 & 0 & 3 of 6 \\
\hline Viable stroma & 0 & 0 & 0 & 0 & 0 & 2 of 3 \\
\hline \% HV conidia & - & - & - & - & - & $18 \%$ \\
\hline Ratings of Asexual Reproduction After 12 Months: CHV1-Ep713 \\
\hline Anastomosed & Bockenhauer & Schomberg & Euro7ssv & JR10 & WR2 & Ep155 \\
\hline Stroma Rating & 0.06 & 0 & 0 & NA & 0 & 0 \\
\hline \# of cankers & 2 of 7 & 0 & 0 & & 0 & 0 \\
\hline Viable stroma & 1 of 2 & 0 & 0 & & 0 & 0 \\
\hline \% HV & $\mathbf{4 8} \%$ & - & - & & - & - \\
\hline Transfected & Bockenhauer & Schomberg & Euro7ssv & JR10 & WR2 & Ep155 \\
\hline Stroma Rating & 0 & 0 & 0 & 0 & NA & 0 \\
\hline \# of cankers & 0 & 0 & 0 & 0 & & 0 \\
\hline Viable stroma & 0 & 0 & 0 & 0 & & 0 \\
\hline \% HV & - & - & - & - & & - \\
\hline
\end{tabular}

Table 10. Average stroma rating and percentage of $\mathrm{HV}$ transmission to conidia from CHV1-Ep713 isolates rated and sampled after 4 (11/12/03) and 12 months (7/29/04).

\section{Asexual Reproduction and HV Transmission after 5 and 12 Months:}

\section{CHV3-County Line}

When cankers were rated and sampled in November 2003, isolates infected with CHV3-County Line sporulated at rates similar to those infected with CHV1-Ep713infected hypovirus and significantly less than those infected with CHV1-Euro7 $(\mathrm{P}=0.003)$ (Figure 19). 


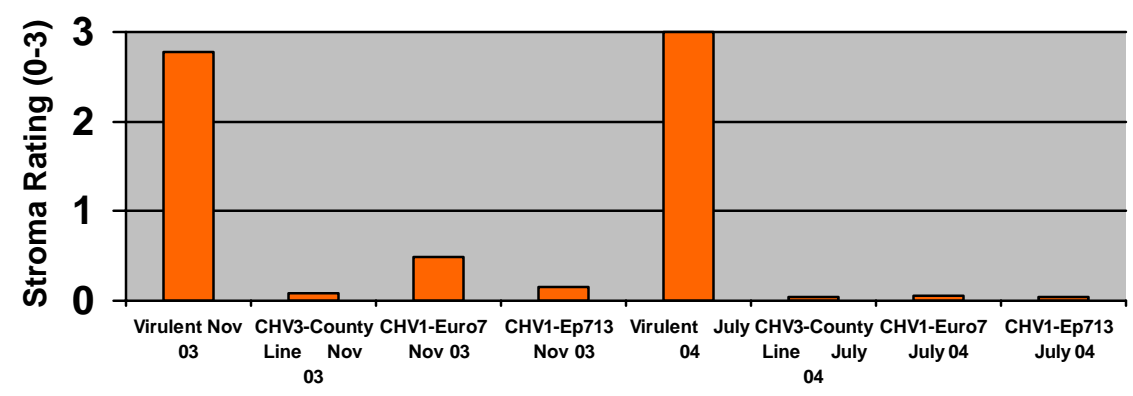

Figure 19. Average stroma produced by all anastomosed isolates when cankers were rated after 4 (11/12/03) and 12 months (7/29/04). CHV1-Euro7 and CHV1-Ep713 data are repeated from Figure 17. CHV1-Euro7 infected isolates produced significantly more stroma in November than CHV1-Ep713 or CHV3-County Line.

\begin{tabular}{|c|c|c|c|c|c|c|}
\hline \multicolumn{7}{|c|}{ Ratings of Asexual Reproduction After 4 months: CHV3-County Line } \\
\hline Anastomosed & Bockenhauer & Schomberg & Euro7ssv & JR10 & WR2 & Ep155 \\
\hline Stroma Rating & 0 & 0 & 0.43 & 0.17 & 0 & 0 \\
\hline \# of cankers & 0 & 0 & 3 of 7 & 1 of 6 & 0 & 0 \\
\hline Viable stroma & 0 & 0 & 3 of 3 & 0 & 0 & 0 \\
\hline$\% \mathrm{HV}$ & - & - & $8 \%$ & - & - & - \\
\hline \multicolumn{7}{|c|}{ Ratings of Asexual Reproduction After 12 Months: CHV3-County Line } \\
\hline Anastomosed & Bockenhauer & Schomberg & Euro7ssv & JR10 & WR2 & Ep155 \\
\hline Stroma Rating & 0 & 0.17 & 0.06 & 0 & 0 & 0 \\
\hline \# of cankers & 0 & 1 of 6 & 3 of 7 & 0 & 0 & 0 \\
\hline Viable stroma & 0 & 1 of 1 & 3 of 3 & 0 & 0 & 0 \\
\hline$\% \mathrm{HV}$ & - & $2 \%$ & $16 \%$ & - & - & - \\
\hline
\end{tabular}

Table 11. Average stroma rating and percentage of $\mathrm{HV}$ transmission to conidia from CHV3-County Line isolates rated and sampled after 4 (11/12/03) and 12 months (7/29/04). 


\section{Comparison of HV Transmission to Conidia in vitro}

In contrast to the field results, the test isolates produced abundant stroma containing viable conidia when cultured on PDA in the laboratory. The percentage of hypovirus transmission to conidia when isolates were examined collectively was $83 \%$ and 88\% for anastomosed and transfected isolates, respectively (Figure 20). This difference was not statistically significant $(\mathrm{P}=0.37)$.

No statistical differences between anastomosed and transfected isolates were detected when hypovirus transmission between CHV1-Euro7 and CHV1-Ep713-infected isolates were compared (Table 12). When contrasting fungal isolates, Schomberg consistently gave high transmission rates regardless of the CHV1 hypovirus.

Bockenhauer and Euro7ssv genomes generated a higher percent $\mathrm{HV}$ transmission when infected with CHV1-Ep713 than CHV1-Euro7. In contrast, WR2 and JR10 produced dramatically higher percentages of CHV1-Euro7 infected conidia (Table 12).

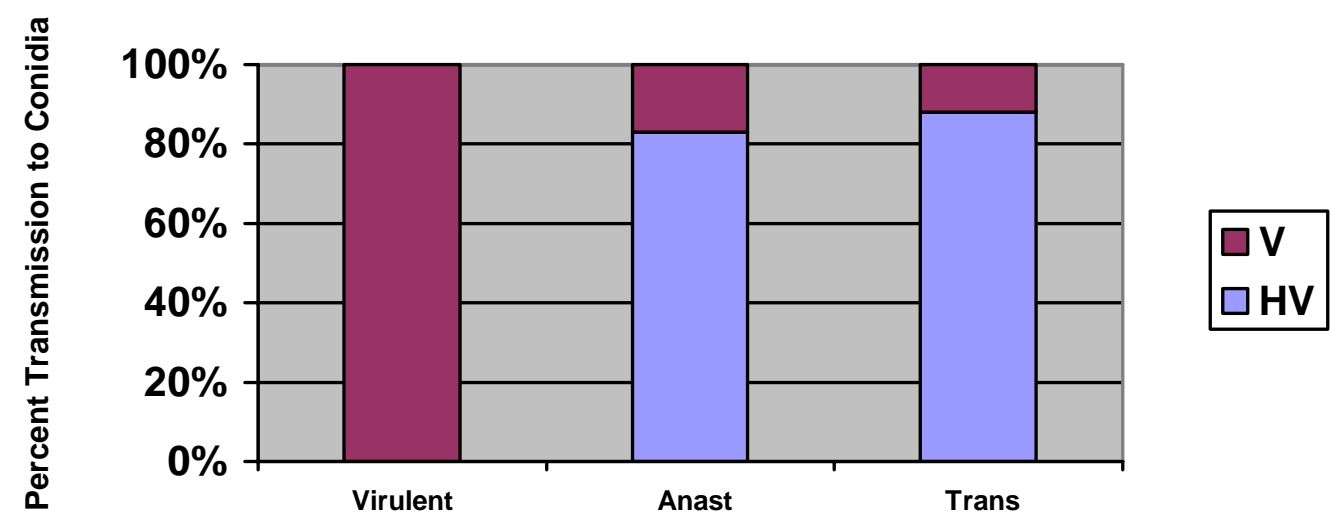

Figure 20. Percentage of hypovirulent (HV) and virulent (V) colonies resulting from single-conidial isolations cultured in vitro. No differences existed between anastomosed and transfected isolates when isolates were compared over all treatment groups. 


\begin{tabular}{|c|c|c|c|c|c|}
\hline \multicolumn{6}{|c|}{ Hypovirus Transmission to Conidia: CHV1-Euro7 } \\
\hline \multicolumn{3}{|c|}{ Anastomosed } & \multicolumn{3}{|c|}{ Transfected } \\
\hline $\mathrm{t}$ & Parental Background & Mean & $\mathrm{t}$ & Parental Background & Mean \\
\hline A & Schomberg & $99 \%$ & A & JR10 & $99 \%$ \\
\hline A & WR2 & $95 \%$ & A & WR2 & $98 \%$ \\
\hline A B & JR10 & $84 \%$ & A B & Schomberg & $92 \%$ \\
\hline A B & Bockenhauer & $83 \%$ & A B & Euro7ssv & $85 \%$ \\
\hline \multirow[t]{2}{*}{ B } & Euro7ssv & $73 \%$ & B & Bockenhauer & $81 \%$ \\
\hline & Ep155 & NA & & Ep155 & NA \\
\hline \multicolumn{6}{|c|}{ Hypovirus Transmission to Conidia: CHV1-EP713 } \\
\hline \multicolumn{3}{|c|}{ Anastomosed } & \multicolumn{3}{|c|}{ Transfected } \\
\hline $\mathrm{t}$ & Parental Background & Mean & $\mathrm{t}$ & Parental Background & Mean \\
\hline A & Schomberg & $100 \%$ & A & Schomberg & $100 \%$ \\
\hline A & Euro7ssv & $94 \%$ & A & Bockenhauer & $99 \%$ \\
\hline A & Bockenhauer & $93 \%$ & A & Euro7ssv & $97 \%$ \\
\hline A & Ep155 & $91 \%$ & A & Ep155 & $92 \%$ \\
\hline $\mathrm{B}$ & WR2 & $23 \%$ & B & JR10 & $37 \%$ \\
\hline & JR10 & NA & & WR2 & NA \\
\hline
\end{tabular}

Table 12. Tukey's HSD test comparing the percentage of HV transmission to progeny between CHV1-Euro7 and CHV1-Ep713 anastomosed and transfected isolates. Means designated by the same letter are not significantly different. No significant differences existed between anastomosed and transfected isolates.

\section{Comparison of HV transmission to Conidia in vitro among CHV3-County Line, CHV1-Euro7, and CHV1-Ep713 Hypoviruses}

When anastomosed isolates were collectively compared, those infected with CHV3-County Line generated significantly fewer HV conidia than isolates infected with CHV1-Euro7 ( $\mathrm{P}=0.0001)$ or CHV1-Ep713 (0.005). The means were 87\% CHV1Euro7, 73\% CHV1-Ep713, and 49\% CHV3-County Line (Figure 21). There were significant 2-way interactions among the parental backgrounds and the three hypoviruses $(\mathrm{P}=0.001)$. Transmission among the parental backgrounds infected with CHV3-County Line was significantly lower with few exceptions (Table 13). 


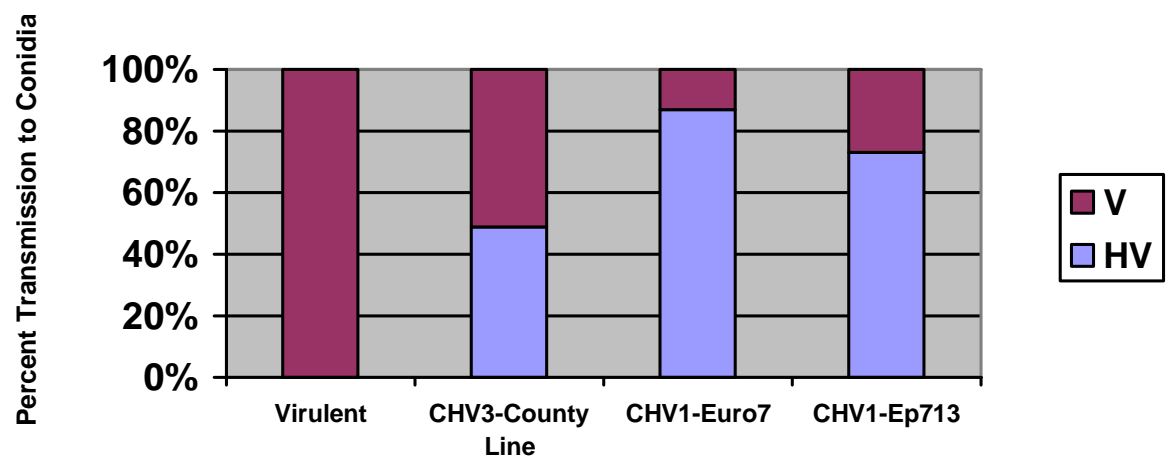

Figure 21. Comparison of the percentage of hypovirulent (HV) and virulent (V) colonies resulting from single-conidial isolation from anastomosed isolates cultured in vitro. CHV3-County Line isolates had a significantly lower transmission rate when compared to CHV1-hypovirus infected isolates. CHV1Euro7 and CHV1-Ep713 data are repeated from Figure 20.

\begin{tabular}{|l|l|l|l|l|l|l|l|l|}
\hline \multicolumn{9}{|c|}{ Hypovirus Transmission to Conidia } \\
\hline \multicolumn{3}{|c|}{ CHV3-County Line } & \multicolumn{3}{c|}{ CHV1-Euro7 } & \multicolumn{4}{|c|}{ CHV1-Ep713 } \\
\hline t & Background & $\mathbf{9}$ & t & Background & $\mathbf{\%}$ & t & Background & $\mathbf{\%}$ \\
\hline A & WR2 & $\mathbf{9 1}$ & A & Schomberg & $\mathbf{9 9}$ & A & Schomberg & $\mathbf{1 0 0}$ \\
\hline AB & Euro7ssv & 61 & A & WR2 & $\mathbf{9 5}$ & A & Euro7ssv & $\mathbf{9 4}$ \\
\hline ABC & JR10 & $\mathbf{4 9}$ & AB & JR10 & $\mathbf{8 4}$ & A & Bockenhauer & $\mathbf{9 3}$ \\
\hline BC & Schomberg & $\mathbf{3 4}$ & AB & Bockenhauer & $\mathbf{8 3}$ & A & Ep155 & $\mathbf{9 1}$ \\
\hline BC & Bockenhauer & $\mathbf{3 2}$ & B & Euro7ssv & 73 & B & WR2 & $\mathbf{2 3}$ \\
\hline C & Ep155 & $\mathbf{2 6}$ & & Ep155 & NA & & JR10 & NA \\
\hline
\end{tabular}

Table 13. Tukey's HSD test comparing CHV3-County Line infected isolates. The percentage of HV transmission to progeny among parental backgrounds when single conidia were isolated from cultures grown on PDA was measured. Means designated by the same letter are not significantly different. CHV1-Euro7 and CHV1-Ep713 data are repeated from Table 12. Percentages in bold text indicate significant differences among hypoviruses.

\section{Hypovirus Transmission via Anastomosis in vitro}

Pairings were made in vitro to attempt hypovirus transmission via anastomosis between vegetative incompatible isolates used in this study. Pairings between isogenic (compatible) V and HV isolates served as controls. When data were pooled, anastomosed and transfected isolates performed the same in terms of rate of hypovirus transmission; 22\% and 20\%, respectively $(\mathrm{P}=0.17)$ (Figure 22). 


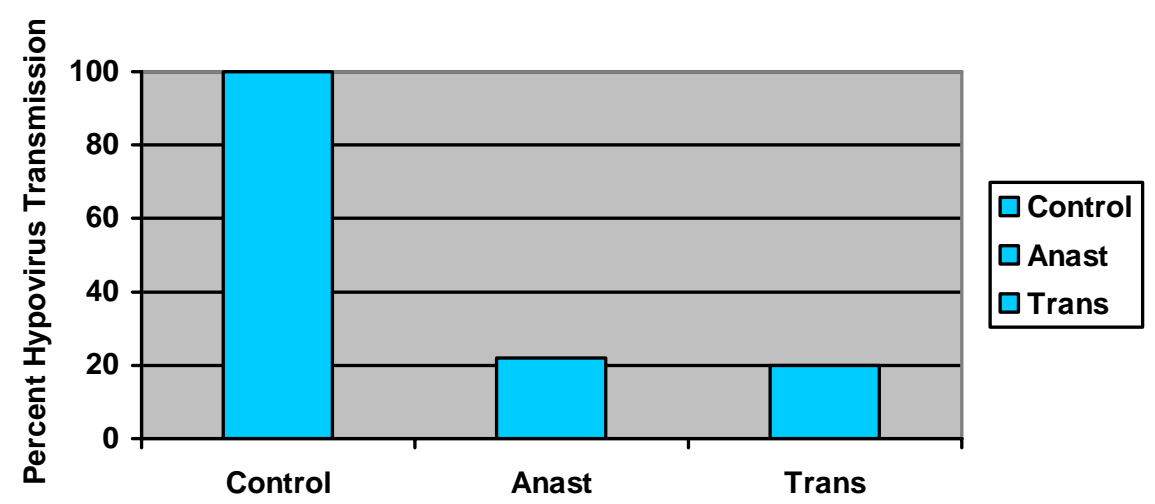

Figure 22. The percentage of hypovirus transmission that resulted from pairing incompatible isolates over all treatment groups. No differences between anastomosed and transfected isolates were noted

\section{HV Transmission among Parental Backgrounds}

When hypovirus transmission between isolate combinations were compared, transmission rates depended on the genome of the fungus and was not influenced by the mode of infection or the infecting hypovirus. One example of HV transmission that was observed between incompatible isolates occurred when Euro7ssv was hypovirus-infected, it transmitted its hypovirus to virulent WR2 isolate during each pairing (Table 14). This trend also was observed when the pairings were reversed; virus-containing WR2 isolate paired with the Euro7ssv virulent isolate. In contrast, when hypovirulent JR10 isolates was paired with the Schomberg virulent isolate, hypovirus transmission occurred each time. However, hypovirulent Schomberg isolates did not transmit hypovirus to JR10 when it was the virulent recipient (Table 14). 


\begin{tabular}{|c|c|c|c|c|c|c|c|c|c|c|c|c|}
\hline \multirow{3}{*}{ HV Isolate } & \multicolumn{12}{|c|}{ HV Transmission } \\
\hline & \multicolumn{2}{|c|}{$\begin{array}{c}\text { Bock } \\
\text { Virulent }\end{array}$} & \multicolumn{2}{|c|}{$\begin{array}{l}\text { Schom } \\
\text { Virulent }\end{array}$} & \multicolumn{2}{|c|}{$\begin{array}{l}\text { Euro7ssv } \\
\text { Virulent }\end{array}$} & \multicolumn{2}{|c|}{$\begin{array}{c}\text { JR10 } \\
\text { Virulent }\end{array}$} & \multicolumn{2}{|c|}{$\begin{array}{c}\text { WR2 } \\
\text { Virulent }\end{array}$} & \multicolumn{2}{|c|}{$\begin{array}{c}\text { Ep155 } \\
\text { Virulent }\end{array}$} \\
\hline & $\mathbf{A}$ & $\mathbf{T}$ & $\mathbf{A}$ & $\mathbf{T}$ & A & $\mathbf{T}$ & $\mathbf{A}$ & $\mathbf{T}$ & $\mathbf{A}$ & $\mathbf{T}$ & $\mathbf{A}$ & $\mathbf{T}$ \\
\hline Bock(Euro7) & 5 & 5 & 2 & 0 & 0 & 0 & 0 & 0 & 0 & 0 & 2 & 1 \\
\hline Bock(Ep713) & 5 & 5 & 0 & 0 & 0 & 0 & 0 & 0 & 0 & 0 & 0 & 0 \\
\hline Bock(Coli) & 5 & NA & 0 & NA & 0 & NA & 0 & NA & 0 & NA & 1 & NA \\
\hline Schom(Euro7) & 1 & 0 & 5 & 5 & 2 & 2 & 1 & 0 & 0 & 1 & 3 & 3 \\
\hline Schom(Ep713) & 2 & 1 & 5 & 5 & 3 & 1 & 0 & 0 & 0 & 0 & 3 & 2 \\
\hline Schom(Coli) & 3 & NA & 5 & NA & 0 & NA & 0 & NA & 0 & NA & 0 & NA \\
\hline E7ssv(Euro7) & 1 & 0 & 0 & 1 & 5 & 5 & 0 & 0 & 5 & 5 & 1 & 0 \\
\hline E7ssv(Ep713) & 0 & 0 & 0 & 0 & 5 & 5 & 2 & 2 & 5 & 5 & 0 & 1 \\
\hline E7ssv(Coli) & 3 & NA & 1 & NA & 5 & NA & 3 & NA & 5 & NA & 0 & NA \\
\hline JR10(Euro7) & 1 & 1 & 5 & 5 & 0 & 0 & 5 & 5 & 0 & 0 & 1 & 2 \\
\hline JR10(Ep713) & NA & 0 & NA & 5 & NA & 1 & NA & 5 & NA & 1 & NA & 1 \\
\hline JR10(Coli) & 1 & NA & 5 & NA & 1 & NA & 5 & NA & 0 & NA & 0 & NA \\
\hline WR2(Euro7) & 0 & 0 & 0 & 0 & 5 & 5 & 0 & 1 & 5 & 5 & 0 & 1 \\
\hline WR2(Ep713) & 0 & NA & 0 & NA & 5 & NA & 0 & NA & 5 & NA & 0 & NA \\
\hline WR2(Coli) & 0 & NA & 0 & NA & 5 & NA & 1 & NA & 5 & NA & 0 & NA \\
\hline Ep155(Euro7) & NA & NA & NA & NA & NA & NA & NA & NA & NA & NA & NA & NA \\
\hline Ep155(Ep713) & 1 & 0 & 0 & 0 & 0 & 0 & 2 & 1 & 0 & 0 & 5 & 5 \\
\hline Ep155(Coli) & 1 & NA & 0 & NA & 0 & NA & 2 & NA & 0 & NA & 5 & NA \\
\hline
\end{tabular}

Table 14. Results for hypovirus transmission of virulent isolates (top row) when paired with anastomosed (A) and transfected (T) HV isolates (left hand column). Number reported is the number of times HV transmission occurred out of 5 pairings. $\mathrm{NA}=$ Not available for this study.

Two isolates, JR10 and Euro7ssv, were able to transmit their hypoviruses among the greatest number of isolates (Tables 15, 16). Schomberg, WR2, and Ep155 isolates were intermediate in this ability and Bockenhauer consistently had the lowest rate of hypovirus transmission. These trends generally remained consistent regardless of the infecting hypovirus (Table 16). 


\begin{tabular}{|l|l|l|l|l|l|}
\hline \multicolumn{7}{|c|}{ Aypovirus Transmission: CHV1-Euro7 Isolates } \\
\hline t & Parental Background & \#Conv. & T & Parental Background & \#Conv. \\
\hline A & Euro7ssv & 8 & A & JR10 & 8 \\
\hline A & JR10 & 7 & A & WR2 & 7 \\
\hline A & Schomberg & 7 & A & Euro7ssv & 7 \\
\hline A & WR2 & 5 & A & Schomberg & 5 \\
\hline A & Bockenhauer & 4 & A & Bockenhauer & 1 \\
\hline \multicolumn{7}{|c|}{ Hypovirus Transmission: CHV1-Ep713 Isolates } \\
\hline \multicolumn{7}{|c|}{ Anastomosed } & \multicolumn{4}{c|}{ Transfected } \\
\hline t & Parental Background & \#Conv & T & Parental Background & \# Conv \\
\hline A & Schomberg & 8 & A & Euro7ssv & 8 \\
\hline A B & Euro7ssv & 5 & A & JR10 & 8 \\
\hline A B & WR2 & 5 & A B & Schomberg & 4 \\
\hline A B & Ep155 & 3 & A B & Ep155 & 1 \\
\hline B & Bockenhauer & 0 & B & Bockenhauer & 0 \\
\hline & JR10 & NA & & WR2 & NA \\
\hline
\end{tabular}

Table 15. Chi-square test contrasting frequency of hypovirus transmission to incompatible isolates infected with CHV1-Euro7 or CHV1-Ep713. Isolates connected by the same letter are not significantly different. Number of conversion (\# Conv) is 5 incompatible pairings replicated 5 times (25 pairings total).

\begin{tabular}{|c|c|c|}
\hline \multicolumn{3}{|c|}{$\begin{array}{l}\text { Hypovirus Transmission: } \\
\text { CHV3-County Line Isolates }\end{array}$} \\
\hline \multicolumn{3}{|c|}{ Anastomosed } \\
\hline $\mathrm{t}$ & Parental Background & \# Conv \\
\hline A & Euro7ssv & 12 \\
\hline A B & JR10 & 7 \\
\hline A $B$ & WR2 & 6 \\
\hline B & Schomberg & 3 \\
\hline B & Ep155 & 1 \\
\hline B & Bockenhauer & 1 \\
\hline
\end{tabular}

Table 16. Chi-square test contrasting horizontal transmission between vegetative incompatible isolates infected with CHV3-County Line. Isolates connected by the same letter are not significantly different. Number of conversion (\# Conv) is 5 incompatible pairings replicated 5 times (25 pairings total). 


\section{Discussion}

Superficial cankers were observed on European chestnuts in Italy less than twenty years after the accidental introduction of C. parasitica. Chestnut blight has diminished in some regions of Europe, presumably due to the natural biological control caused by hypoviruses (Rigling et al., 1989). This recovery from blight now is known to be associated with dsRNA viruses mainly in the family Hypoviridae (Hillman and Suzuki, 2004). Unfortunately, hypovirulence-mediated biological control has been far less successful in North America. This may be due in part from the diversity in vegetative compatibility among C. parasitica populations that inhibit hypovirus transmission between incompatible isolates (Milgroom and Cortesi 2004). There are other factors that may have impeded the success of hypovirulence in North America. European chestnuts are not as susceptible to the blight as American chestnuts (Hebard et al., 1984). The partial resistance of European chestnut may allow more time for hypovirulent isolates to transmit their hypoviruses to virulent strains thereby establishing hypovirus infection, preventing tree mortality. In addition, sanitation practices as well as the removal of competing vegetation in orchards in Europe may reduce stress and promote tree growth (Milgroom and Cortesi 2004). Implementing such management practices in the forests of North America would not be economically feasible even if hypovirulent strains were present. An additional factor that presumably can reduce the effectiveness of hypovirulent isolates is the reduction of asexual spore production. Even though hypovirus can be transmitted to conidia, the sporulation of $\mathrm{HV}$ isolates does not rival wild-type strains. Furthermore, hypovirulent isolates lack the ability to reproduce 
sexually. Therefore any ascospores that are produced are virulent and maintain the virulence of the fungus and the diversity of vegetative compatibility types.

A field isolate that has the ability to produce non-lethal cankers, persist in the forest, generate hypovirulent inoculum, and transmit hypovirus to a variety of vegetative compatibility types presumably would aid in the successful use of hypoviruses as biological control agents. Studies that analyze the fungus have been conducted with intention of selecting isolates that display these characteristics. Anastomosis-mediated transfer of hypoviruses commonly is used to study the performance of a variety of fungal and hypovirus genome combinations. However, infecting vegetative incompatible isolates with different hypoviruses often fails despite repeated attempts to do so on artificial medium or in chestnut bark.

Transfection is an alternative laboratory method of hypovirus transmission that can be used to create hypovirulent strains. This technique involves the insertion of a synthetic hypovirus dsRNA into individual fungal spheroplasts by electroporation. The spheroplasts are regenerated on specialized media and the successfully transfected colonies can be chosen based on phenotype. A transfected strain has the same phenotypic traits as a strain infected via anastomosis. Previous studies assessing growth and sporulation on excised stems (Chen and Nuss 1999) and HV persistence on chestnut sprouts in the field (Double, unpublished data) have concluded that transfected isolates behave comparably to their anastomosed counterparts in terms of pathogenicity and $\mathrm{HV}$ persistence.

The objective of this research was to conduct a second field study to further document whether hypovirulence is due solely to the acquisition of the dsRNA or if the 
other cytoplasmic elements that may be transferred through the cytoplasm during anastomosis play a role in how the hypoviruses are being expressed. This was accomplished by evaluating the performance of isolates which were infected with hypoviruses either by transfection or anastomosis. Three experiments were conducted. The first test confirmed the growth and HV persistence of both isolate types previously tested by Double in 1998-99. In addition to the original isolates used in this previous field test, another set of isolates containing the CHV3-County Line hypovirus were included. The second component of this research involved both laboratory and field tests to evaluate the quality and quantity of hypovirulent inoculum produced by various isolates. Conidia produced in vitro and in vivo were retrieved, single spored and plated on PDA. The germinating spores were observed and classified based on their morphology. A third laboratory experiment evaluated whether differences existed in hypovirus transmission rates between transfected and anastomosed isolates when paired with virulent isolates representing a variety of vegetative compatibility types.

After four and twelve months in the field the influence of the hypovirus-infection on the growth of isolates was clearly demonstrated. The cankers initiated by virulent isolates produced significantly larger cankers than their HV counterparts. This finding was consistent with previous studies (Elliston, 1977; Peever et al., 2000). When the general performance of anastomosed and transfected isolates was compared, no consistent trends toward larger or smaller cankers was noted. These results were comparable and confirmed the work by Chen and Nuss (1999) on excised stems and the previous field study conducted by Double (per. com.). The parental background appeared to contribute to the differences in the size of cankers produced by the HV 
isolates. The variations in rankings observed among the isolates infected with CHV1Euro7 were in most cases similar to the rankings of the isolates in their virulent form (Tables 2, 5). That is, the most invasive strains, Bockenhauer, Schomberg, and Ep155, generally produced, with some exceptions, significantly larger cankers while JR10 and WR2 produced smaller ones. Euro7ssv isolates produced cankers that were intermediate in size. This too was observed in the previous studies (Chen and Nuss, 1999; Double, unpublished data) indicating that the fungal host may play a role in how the fungus responds to hypovirus infection.

When comparing isolates that were infected with CHV1-Ep713 to those with CHV1-Euro7, there were no significant differences in size of cankers produced when all virus-infected isolates were pooled. However, when individual isolates were compared two notable exceptions existed; Schomberg(CHV1-Euro7) anastomosed and Bockenhauer(CHV1-Euro7) transfected produced cankers significantly larger than their CHV1-Ep713 counterparts. This was in contrast to the 1998 field study where the only significant difference was when larger cankers were produced by the Ep155(CHV1Euro7) isolate when compared to those initiated by Ep155(CHV1-Ep713) (Double per. com.). Unfortunately, in the current test the Ep155(CHV1-Euro7) isolate apparently lost its hypovirus and grew as a virulent isolate, thus data were not available for comparison. Other field tests have reported a more aggressive growth habit for CHV1-Euro7-infected isolates compared to the debilitated expansion observed from those infected with CHV1Ep713 (Double and MacDonald, per. com.). Chen and Nuss (1999) also reported larger cankers initiated by Ep155 and Euro7ssv when infected with CHV1-Euro7 hypovirus when compared to CHV1-Ep713 infected isolates. This is in contrast to this field study 
where the fungal isolate appeared to play a greater role in how these hypoviruses were expressed. For example, Euro7ssv isolates produced cankers similar in size regardless of the infecting CHV1 hypovirus. In contrast, isolates JR10 and WR2, when infected with the CHV1-Euro7, produced smaller cankers than their CHV1-Ep713 counterparts. Thus, CHV1-Euro7 hypovirus may not be a suitable biological control agent when present in some fungal backgrounds. This would indicate that the isolate plays a very important role in hypovirus expression and thus how well suited particular hypovirus/isolate combinations are as biological control agents.

When CHV3-County Line was included as a comparative hypovirus, isolates infected with this virus produced significantly smaller cankers than the CHV1hypoviruses when assessed after either 4 or 12 months of growth (parental backgrounds pooled). Furthermore, all fungal backgrounds produced cankers similar in size when infected with CHV3-County Line. Isolates that produced larger cankers were severely affected by the CHV3-County Line hypovirus. The significant debilitation caused by the CHV3-type hypovirus has been observed in previous studies (Milgroom and Cortesi 2004; Double, per. com.). CHV1 and CHV3-type hypoviruses differ genetically (Hillman and Suzuki, 2004) and it appears that CHV3-County Line hypovirus significantly down regulates of genes responsible for pathogenesis of its fungal host. Persistence of hypoviruses within the cankers was analyzed by the removal of bark plugs at the time the cankers were measured (4 and 12 months after initiation). As the cankers aged during this study, the percentage of hypovirulent isolates recovered increased. This occurred regardless of hypovirus-infection method, parental background, or hypovirus with the exception of Ep155 infected with CHV1-Ep713. Presumably, 
seasonal differences between sampling periods may have influenced the increase in recovery of hypovirulent strains. Undoubtedly the fungus was growing more actively in July than November and the hypovirus may have been more actively replicating resulting in better recovery rates of hypovirus infected strains.

When the two methods of hypovirus infection were compared, no consistent differences in the recovery of $\mathrm{HV}$ isolates existed between anastomosed and transfected isolates when data were pooled. However, differences in recovery were observed among individual parental backgrounds. Essentially, cankers which enlarged the most (Schomberg, Bockenhauer, and Ep155) generally yielded the highest percentage of hypovirulent isolates (Tables 3, 4, 6, 7). When the CHV3-County Line isolates were included for comparison (parental backgrounds pooled) the recovery of the two CHV1types was significantly higher than CHV3-County Line after 4 months in the field. This could be due to the limited area of bark invaded due to reduced fungal activity of CHV3County Line infected isolates. As previously discussed, larger cankers generally yielded higher hypovirus recovery rates. However, after 12 months the HV recovery was similar among the three hypoviruses (parental backgrounds pooled). Therefore, it can be speculated that CHV3-County Line isolates are slower to establish, however, after they do, they persist comparably to CHV1-hypoviruses. Thus, over time HV isolate recovery does not appear to be dependent on the method of hypovirus acquisition or the hypoviruses used. Rather, hypovirus persistence appears to be based on how successfully certain fungal/hypovirus combinations can establish themselves in the bark of their host tree (Tables 6, 7, 8). 
Hypovirulent cultures of $C$. parasitica are generally stable in culture (Double and MacDonald, per. com; Hillman and Suzuki, 2004) and consequently, should retain their hypoviruses in cankers. Therefore it is curious that virulent colonies were recovered from cankers initiated by $\mathrm{HV}$ isolates. Some virulent isolates that were recovered were vegetatively incompatible with the test isolate. This indicated that the virulent strain that was recovered was not the $\mathrm{HV}$ isolate used to establish the canker. One explanation could be that wounding during canker initiation (Kuhlman, 1983) or during the period of canker expansion provided an opportunity for wild-type C. parasitica to become established in the same canker. Some virulent colonies isolated from cankers were vegetatively compatible with the infecting strain when vc tested. These compatible isolates may coincidentally have been the same vc type. A second explanation may be that virulent hyphae developed from the infecting hypovirulent strain. The stability of hypovirulent isolates in bark has not been studied. The bark may provide a substrate that stimulates virulent hyphal growth. Since hypovirulent hyphae do not colonize host tissue as well, virulent hyphae may have outgrown the hypovirus-infected thallus.

As the percentage of $\mathrm{HV}$ isolates that were recovered during the summer sampling period increased the percentages of the contaminants decreased. The majority of contaminants consisted of Aspergillus spp., Penicillium spp., Pestilotia spp., and Trichoderma spp; all fairly common fungi that have been associated with infected chestnut bark (Jones, 2003). This decrease in the number of contaminants was in contrast to two previous field studies where the number of other organisms isolated increased when the percentage of HV increased (Bell, 2004; Jones 2003). However, in both these studies, cankers were initiated by virulent isolates then treated with HV inoculum. A 
much larger canker initially was produced in these studies. As a result, there was a larger area of dead tissue (bark) potentially generating a greater substrate for growth of other organisms. In this study, HV isolates were used to initiate infections that produced superficial cankers with less necrotic tissue. This artificial means of canker initiation may not be what occurs in nature, as it is not known whether hypovirulent infections can establish naturally. In areas where the phenomenon of hypovirulence provides active biological control, non-lethal hypovirulent cankers may be the result of virulent cankers that acquired the hypoviruses via anastomosis. Thus, larger virulent cankers that are established first may provide an increase in necrotic tissue resulting in an increase in colonization by other saprophytic microorganisms. Furthermore, the level of contaminants that exist in naturally occurring cankers has not been studied extensively as well as whether these microorganisms contribute to biological control. These other organisms may be antagonistic to C. parasitica contributing to biological control in areas where hypoviruses are established (Minervini and Bisiach, 1993).

Stroma production by HV isolates was poor compared to virus-free isolates, as has been noted in previous studies (Elliston, 1985; MacDonald and Fulbright, 1991; Peever et al., 2000). All virulent isolates sporulated prolifically and trees harboring virulent isolates did not produce callous; a finding typical of most virulent infections. In contrast, only $31 \mathrm{HV}$ cankers produced stroma in November 2003 and only 10 when rated in July 2004. The decline in sporulation observed between sampling periods is undoubtedly due to poor growth of the HV isolates. However, host response may be a factor. The attenuation in virulence caused by hypovirus-infection may have provided the host tree ample time to activate its defense mechanisms. Therefore the HV isolates 
may have been further debilitated by host responses within an actively growing tree. When rated in November the host tree was dormant and may have been incapable of responding to infection. However, in July the tree was actively growing and capable of producing polyphenolic compounds (McCarroll and Thor, 1978), ethylene (Hebard and Shain, 1988), and callous tissue. Therefore host responses may further diminish spore production by hypovirulent isolates and be dependent on the season of infection.

There were no differences between anastomosed and transfected isolates in terms of stromata production during either sampling period. The isolates that produced stroma were the isolates that also were the most pathogenic (Bockenhauer, Schomberg, Ep155 and Euro7ssv). Previous studies have shown stroma production to be dependent on the fungal genome (Ellison, 1977; Ellison, 1985; Chen and Nuss, 1999). CHV1-Euro7infected isolates produced more stroma than CHV1-Ep713 and CHV3-County Line infected isolates when rated in November 2003. Although CHV1-Euro7 infected isolates produced more stroma than CHV1-Ep713 and CHV3-County Line infected isolates, the amount of stroma produced by isolates infected with CHV1-Euro7 paled in comparison to their isogenic virulent counterparts. Greater sporulation from CHV1-Euro7 infected isolates previously has been observed on excised stems (Chen and Nuss 1999) and chestnut stems in the field (Hillman and Suzuki, 2004; Double and MacDonald per. com.), however this was not observed during this study. Further evidence for the lack of sporulation from CHV1-Euro7 infected isolates was better illustrated when cankers were rated in July 2004. Sporulation was so poor at this time that CHV1-Euro7, CHV1Ep713, and CHV3-County Line infected isolates were similar in their lack of spore production. 
The production of hypovirulent conidia in the forest ecosystem helps maintain the natural spread of hypoviruses and would seem to be a necessary prerequisite for successful biological control. The low rate at which HV isolates sporulate would appear to be a major barrier impeding the success of hypovirulence as a biological control agent. As mentioned previously, the amount of stroma produced by hypovirulent isolates during this study was extremely low. Therefore, the spread of hypovirus via conidia presumably would be dramatically limited, regardless of the method by which the isolates were hypovirus infected. This field study emphasized the competitive disadvantage HV isolates have in the field compared to the virulent wild-types. Hypovirulent isolates may have the ability to retard canker development, however, the lack dissemination via ascospores or conidia makes it impossible for HV isolates to rival the inoculum levels produced by virulent cankers.

Four of the cankers sampled in July 2004 produced all virulent colonies when single-conidial isolations were performed. The absence of HV colonies was suspicious because a percentage of the conidia should contain hypovirus. This prompted a vegetative compatibility test to confirm infecting strain. All 4 virulent isolates in question were vegetatively incompatible with the test isolate used to initiate the canker indicating that the stroma sampled was not produced by the HV test isolate. The stroma collected from non-related virulent isolates demonstrated the occurrence of secondary infections presumably by wild-type C. parasitica; a finding discussed previously with regard to HV recovery. This exemplifies how cankers can quickly become a complex assortment of infecting $C$. parasitica strains. This situation is further complicated by the barriers imposed by vegetative incompatibility. In nature, this type of secondary virulent 
infection could girdle the part of the tree where the infection was established despite the presence of hypovirulent isolates.

In the laboratory, single-conidial isolations were performed to compare hypovirus transmission rates in vitro to rates observed in the field study. There were no significant differences when comparing the rate of hypovirus transmission to conidia between anastomosed and transfected isolates. Isolates that acquired the hypovirus by either method performed similarly. CHV1-infected isolates have been reported to transmit their hypoviruses at rates ranging from 90-100\% (Milgroom and Cortesi, 2004; Hillman and Suzuki, 2004). During this study CHV1-Euro7 and CHV1-Ep713 infected isolates had similar transmission rates ranging from $80-100 \%$. There were a few exceptions among the CHV1 infected isolates where certain backgrounds generated unusually low hypovirus transmission rates (Table 12). This indicates that HV transmission to conidia may depend on the fungal host; a trait noted in previous single-conidial isolations (Elliston, 1985; Peever et al., 2000). CHV3-County Line had a significantly lower rate of hypovirus transmission to conidia when isolates were pooled and compared to CHV1Euro7 and CHV1-Ep713. When examining fungal genomes, CHV3-County Line transmission percentages had a wider variation in rankings among parental backgrounds than those infected with CHV1-Euro7 or CHV1-Ep713 (Table 13). This could be due to the genetics of the different hypoviruses. CHV3 hypoviruses are comprised of one ORF and lacks the protein p40 found on ORF A on the genomes of CHV1 hypoviruses. Further, p40 associated with CHV1 hypoviruses may increase ORF B expression that in turn enhances viral replication increasing dsRNA accumulation and hypovirus transmission (Hillman and Suzuki, 2004). Thus, due to the variation in rankings 
observed among the parental backgrounds infected with CHV3-County Line, it appears that the fungal genome may have some regulatory effects on how well the hypovirus is able to replicate and be passed on during conidiogenesis.

The virulent isolates produced more conidia than the HV isolates when cultured on PDA. All HV isolates cultured on PDA produced an abundance of viable HV conidia which is in stark contrast to what was observed in the field. The difference may be due to inadequate nutrition acquired by the hypovirulent isolate when growing in bark. If the genes responsible for enzymatic activity in the fungus are down regulated due to hypovirus-infection (Hillman and Suzuki, 2004) an HV isolate will lack the ability to activate enzymes required for tissue degradation to obtain nutrients. This may in turn inhibit the formation of fruiting bodies.

Percentage of hypovirus transmission to conidia was significantly higher in the laboratory than in the field. One explanation for this is that when stroma samples were removed from the field cankers and evaluated, stromal pustules may have been produced by both the test isolate and a wild-type $C$. parasitica strain that colonized the same canker. Thus, the conidia isolated from cankers may have been a mixture of conidia from the HV test isolates as well as virulent contaminants. Therefore, the HV percentage of HV conidia produced may have been reduced because conidia produced by wild-type $C$. parasitica were included in the spore counts.

The final experiment in this study involved anastomosis-mediated transfer of hypovirus to evaluate hypovirus transmission between vegetative incompatible isolates. No differences existed between anastomosed and transfected isolates in their capacity to transmit their hypovirus to different vc types. Further, no differences were noted when 
the three hypoviruses were compared. Essentially, the ability to transmit hypovirus when paired in culture depends on the genetics of the isolates being paired. For example, Euro7ssv virulent and WR2 virulent isolates are vegetatively incompatible with each other. However, when Euro7ssv is hypovirus-infected, the isolate readily converted virulent WR2, regardless of the infecting hypovirus (Table 18). This also was true when the pairings were reversed. Vegetative compatible isolates share the same alleles at all vegetative incompatible (vic) loci. If they differ at one or more loci, programmed cell death may occur preventing cytoplasmic transfer reducing horizontal hypovirus transmission (Cortesi et al., 2001). Cortesi found variation among the six vic loci ranging from strong inhibition to no inhibition dependent on the particular loci that were different. He hypothesized that the different vic loci is correlated to the differences in cell death rates. Therefore in this study, the transmission that occurred between WR2 and Euro7ssv may have been the result of delayed cell death allowing for hypovirus transmission. Further, virus transmission may vary depending on which alleles are present in the donor and recipient strain (Milgroom and Cortesi, 2004). For example, when HV JR10 was paired with Schomberg virulent, hypovirus transmission occurred. However, when JR10 was the recipient isolate, a barrage was formed inhibiting hypovirus exchange (Table 14). Tests were not conducted in the forest so it is not known if these transmission rates would occur between these isolates in the field. The diversity of vc types that exist in this country are a major factor hindering hypovirus spread in the North American forests. Therefore, isolates that are able to transmit their hypovirus to a variety of vc types may be better suited for biological control. 
In summary, anastomosis and transfection are valuable techniques that can be used to deliver hypoviruses to a variety of fungal genomes. What is useful about transfection is that it can be a rapid method permitting hypovirus transmission to a broad range of isolates of varying vc types. During this study there were no clear trends that indicated transfected isolates behaved differently with regard to pathogenesis and HV transmission to those that acquired their hypoviruses via anastomosis. These results support previous studies (Chen and Nuss, 1999; Double, unpublished data). Thus, hypovirulence appears to be based solely on the transmission of the dsRNA and not to other components in the cytoplasm that may be transferred into the recipient fungus during anastomosis.

This research also provides additional evidence that each hypovirus interacts with its host differently. The results support previous research that reported varying morphological and pathogenic characteristics dependent on the genome of the hypovirulent isolate and hypovirus (Elliston, 1985; Chen and Nuss, 1999; Peever et al., 2000). The most important message consequential to this study is that certain isolate/hypovirus combinations have better biological control potential than others. It would seem that those fungal isolates that are most pathogenic also have other traits such as increased ability to sporulate and high $\mathrm{HV}$ transmission rates that merit their use as biological control agents. This, however, may not always be the case. For example, certain hypovirus/isolate combinations, JR10 and WR2 infected with CHV1-Euro7, performed very poorly in comparison to the other isolates infected with the same hypovirus. However, these isolates showed an improved biological control potential when harboring CHV1-Ep713. Therefore, hypovirus infection may not create strains that 
are uniform in all biological control characteristics. Rather, it appears to be a complex interaction between fungus and hypovirus genomes resulting in isolates with varying attributes. The implication may be that if hypoviruses can successfully spread in the forest among populations of wild-type $C$. parasitica the result may be a population of isolates that provide the diversity in pathogenicity, sporulation, and $\mathrm{HV}$ transmission as observed in this study. 


\section{Summary}

1. The mode (anastomosis or transfection) by which isolates were infected with hypovirus generally did not influence the size of the cankers that an isolate produced; rather differences in canker size were more dependent on the isolate and the hypovirus it harbored.

2. Isolates representing the six parental backgrounds produced cankers of various sizes when infected by either the two CHV1 hypoviruses. This suggests that the fungal genome influences the degree of hypovirus expression based on the rate of canker expansion.

3. When isolates were infected with the CHV3-County Line hypovirus, no differences were noted in size of the cankers that were produced. This hypovirus severely debilitated all test isolates.

4. The rate of recovery of hypovirulent isolates cultured from bark plugs removed from cankers was not influenced by the mode of the hypovirus infection (anastomosis or transfection). Differences were noted among the hypovirus isolation frequencies of the various isolates. Generally, hypovirulent isolate recovery rates were highest for those that produced the largest cankers and least for isolates that produced smaller infections.

5. Very few cankers, initiated with the hypovirus-infected isolates, produced stromata after one year. This was in sharp contrast to their hypovirus-free counterparts. Some of the stromata that hypovirulent cankers produced yielded only virulent conidia suggesting they were associated with portions of the canker thallus that was virulent.

6. The mode (anastomosis or transfection) of hypovirus transmission to isolates did not influence the rate of transmission to conidia when tested in culture. With few exceptions, hypovirus transmission was very high but also dependent on the parental isolate.

7. There were no significant differences between the anastomosed and transfected isolates with regard to hypovirus transmission when incompatible isolates were paired in culture. 


\section{References}

Anagnostakis, S. L., and Day, P.R. 1979. Hypovirulence conversion in Endothia parasitica. Phytopathology 69: 1226-1229.

Anagnostakis, S. L., and Waggoner, P.E. 1981. Hypovirulence, vegetative incompatibility, and the growth of cankers of chestnut blight. Phytopathology 71: 11981202.

Bell, B. C. 2004. Comparison of chestnut treatment procedures from hypovirus introduction. Master’s Thesis. West Virginia University. Morgantown. 71pp.

Braun, E. L. 1950. Decidiuous forests of Eastern North America. McGraw-Hill Book Co. Inc., New York. 596 pgs.

Chen, B., Choi, G. H., and Nuss, D. 1994. Attenuation of fungal virulence by synthetic infectious hypovirus transcripts. Science 264: 1762-1764.

Chen, B., Chen, C-H., Bowman, B., and Nuss, D. 1996. Phenotypic changes associated with wild-type and mutant hypovirus RNA transfection of plant pathogenic fungi phylogenetically related to Cryphonectria parasitica. Phytopathology 86: 301-310.

Chen, B., and Nuss, D. 1999. Infectious cDNA clone of hypovirus CHV1-Euro 7: a comparative virology approach to investigate virus mediated hypovirulence of chestnut blight fungus Cryphonectria parasitica. Journal of Virology 73: 985-992.

Cortesi, P., and Milgroom, M. G. 1998. Genetics of vegetative incompatibility in Cryphonectria parasitica. Applied and Environmental Microbiology Aug: 2988-2994.

Cortesi, P., McCulloch, C. E., Song, H., Lin, H., and Milgroom, M.G. 2001. Genetic control of horizontal virus transmission in the chestnut blight fungus, Cryphonectria parasitica. Genetics 159: 107-118.

Day, P. R., Dodds, J. A., Ellison, J. E., Jaynes, R. A., and Anagnostakis, S. L. 1977. Double-stranded RNA in Endothia parasitica. Phytopathology 67: 1393-1396.

Day, P.R. 1978. Epidemiology of hypovirulence. p. 118-121. in: Proc. Am. Chestnut Symposium., W.L. MacDonald, F.C. Cech, J. Luchok, and H. C. Smith, eds. West Virginia University Press, Morgantown.

Dodds, J. A. 1978. Double-stranded RNA and Virus-like particles in Endothia parasitica. p. 108-110. in: Proc. Am. Chestnut Symposium., W.L. MacDonald, F.C. Cech, J. Luchok, and H. C. Smith, eds. West Virginia University Press, Morgantown.

Dodds, J. A. 1980. Association of Type 1 Viral-like dsRNA with club-shaped particles in hypovirulent strains of Endothia parasitica. Virology 107: 1-12. 
Elliston, J. E. 1977. Pathogenicity and sporulation of normal and diseased strains of Endothia parasitica in American chestnut. P. 95-100 in: Proc. Am. Chestnut Symposium., W.L. MacDonald, F.C. Cech, J. Luchok, and H. C. Smith, eds. West Virginia University Press, Morgantown.

Elliston, J. E. 1985. Preliminary evidence for two debilitating cytoplasmic agents in a strain of Endothia parasitica from western Michigan. Phytopathology 75: 170-173.

Grente, J., and Berthelay- Sauret, S. 1978. Biological control of chestnut blight. p. 30-34. in: Proc. Am. Chestnut Symposium., W.L. MacDonald, F.C. Cech, J. Luchok, and H. C. Smith, eds. West Virginia University Press, Morgantown.

Griffin, G. J. 1986. Chestnut blight and its control. Horticulture Review 8:291-336.

Hebard, F. V., Griffin, G. J., and Elkins, J. R. 1984. Developmental histopathology of cankers incited by hypovirulent and virulent isolates of Endothia parasitica on susceptable resistant chestnut trees. Phytopathology 74: 140-149.

Hebard, F. V. and Shain, L. 1988. Effects of virulent Endothia parasitica and their metabolites on ethylene production by bark of American and Chinese chestnut and Scarlet oak. Phytopathology 78:841-845.

Hillman, B. I., Halpern, B. T., and Brown, M. P. 1994. A viral dsRNA element of the chestnut blight fungus with a distinct genetic organization. Virology 201: 241-250.

Hillman, B. I. and Suzuki, N. 2004. Viruses of the chestnut blight fungus, Cryphonectria parasitica. Advances in Virus Research. 63: 423-472.

JMP, Version 5. SAS Institute Inc., Cary, NC, 1989-2002.

Jones, W. E. 2003. Recovery of hypovirulent isolates from younger and older Cryphonectria parasitica mycelium in cultures and cankers. Anticipated Master's Thesis. West Virginia University. Morgantown. 80pp.

Kuhlman, E. G. 1978. The devastation of American chestnut by blight. p. 1-3. in: Proc. Am. Chestnut Symposium., W.L. MacDonald, F.C. Cech, J. Luchok, and H. C. Smith, eds. West Virginia University Press, Morgantown.

Kuhlman, E. G. 1983. Effects of Hypovirulence in Cryphonectria parasitica and of secondary blight infections on dieback of American chestnut trees. Phytopathology 73: 1030-1034.

MacDonald, W. L., and Fulbright, D. W. 1991. Biological control of chestnut blight: use and limitations of transmissible hypovirulence. Plant Disease. 75: 656-660. 
McCarroll, D. R. and Thor, E. 1978. Biological control of chestnut blight. pp. 70-72. in: Proc. Am. Chestnut Symposium., W.L. MacDonald, F.C. Cech, J. Luchok, and H. C. Smith, eds. West Virginia University Press, Morgantown.

Milgroom, M. G., MacDonald, W. L., and Double, M. L. 1991. Spatial pattern analysis of vegetative compatibility groups in the chestnut blight fungus, Cryphonectria parasitica. Canadian Journal of Botany. 69: 1407-1413.

Minervini, G. and Bisiach, M. 1993. pp. 485-489. in: Proceedings of the International Congress on Chestnut., E. Antognozzi.

Milgroom, M. G. and Cortesi, P. 2004 Biological control of chestnut blight with hypovirulence: a critical analysis. Annual Review Phytopathology. 42: 311-38.

Mittempergher, L. 1978. The present status of chestnut blight in Italy. p. 34-37. in: Proc. Am. Chestnut Symposium., W.L. MacDonald, F.C. Cech, J. Luchok, and H. C. Smith, eds. West Virginia University Press, Morgantown.

Newhouse, J. R., Hoch, H. C., and MacDonald, W. L. 1983. The ultrastructure of Endothia parasitica. Comparison of a virulent with a hypovirulent isolate. Canadian Journal of Botany. 61: 389-399.

Nuss, Donald L. 1992. Implications of engineered transmissible hypovirulence for biological control of chestnut blight. p. 3-6. in: Proc of the International Chestnut Conference., M. L. Double and W. L. MacDonald, eds. West Virginia University Press, Morgantown.

Paul, C. P., and Fulbright, D. W. 1988. Double-stranded RNA molecules from Michigan hypovirulent isolates of Endothia parasitica vary in size and sequence homology. Phytopathology 78: 751-755.

Peever, T. L., Liu, Y., Cortesi, P., and Milgroom, M. 2000. Variation in tolerance and virulence in the chestnut blight fungus-hypovirus interaction. Applied and Environmental Microbiology 66: 4863-4869.

Rigling, D., Heiniger, U. and Hohl, H. R. 1989. Reduction of laccase activity in dsRNAcontaining hypovirulent strains of Cryphonectria parasitica. Phytopathology. 79: 219223. 


\section{Appendix A: Media Used}

\section{Potato Dextrose Agar (PDA):}

$\begin{array}{lc}\text { Difco }{ }^{\circledR} \text { Potato Dextrose Agar } & 39.0 \mathrm{~g} \\ \text { Methionine } & 0.1 \mathrm{~g} \\ \text { Biotin } & 50 \mu \mathrm{g} \\ \text { Distilled Water } & 1000 \mathrm{ml}\end{array}$

To perform phenol/chloroform extractions for the presence of dsRNA, mycelium plugs were transferred to PDA lined with sterile cellophane.

Glucose Yeast Extract Agar with Antibiotics (GYE/A):

$\begin{array}{lr}\text { Bacto® Dextrose (glucose) } & 10.0 \mathrm{~g} \\ \text { Yeast Extract } & 2.0 \mathrm{~g} \\ \text { KH2PO4 } & 1.0 \mathrm{~g} \\ \text { MgSO4 } & 0.5 \mathrm{~g} \\ \text { Thiamine } & 50 \mu \mathrm{g} \\ \text { Biotin } & 10 \mu \mathrm{g}\end{array}$

Microelements

$\begin{array}{lc}\mathrm{Fe}+++ & 500 \mu \mathrm{g} \\ \mathrm{Mn}++ & 439 \mu \mathrm{g} \\ \mathrm{Zn}++ & 154 \mu \mathrm{g} \\ \text { ® Agar } & 20.0 \mathrm{~g} \\ \text { ed Water } & 1000 \mathrm{ml}\end{array}$

Distilled Water $1000 \mathrm{ml}$ 
Tetracycline Hydrochloride $\quad 100 \mathrm{mg}$

Streptomycin Sulfate $\quad 10 \mathrm{mg}$

Antibiotics were added to cooled media $\left(50^{\circ} \mathrm{C}\right)$.

\section{Brom Cresol Green Agar:}

$\begin{array}{lc}\text { Difco }{ }^{\circledR} \text { Potato Dextrose Agar } & 24.0 \mathrm{~g} \\ \text { Malt Extract } & 7.0 \mathrm{~g} \\ \text { Yeast Extract } & 2.0 \mathrm{~g} \\ \text { Tannic Acid } & 0.8 \mathrm{~g} \\ \text { Brom Cresol Green } & 50.0 \mathrm{mg} \\ \text { Bacto }{ }^{\circledR} \text { Agar } & 13.5 \mathrm{~g} \\ \text { Tween } & 12 \mathrm{drops} \\ \text { Distilled Water } & 1000 \mathrm{ml}\end{array}$

Agar solutions were prepared in $1000 \mathrm{ml}$ flaks and autoclaved at $121 \mathrm{C}$ for 25 minutes. Approximately 25 to $30 \mathrm{ml}$ of the media was dispensed into 100 x $15 \mathrm{~mm}$ plastic Petri dishes. Agar slants were made by steaming GYE/A media for 30minutes, dispensing 7ml aliquots into 125 x 16 mm screw cap test tubes at $121 \mathrm{C}$ for 15 minutes. 


\section{Appendix B: Peptone Solution}

$\begin{array}{ll}\text { Difco }{ }^{\circledR} \text { Bacto-Peptone } & 1.0 \mathrm{~g} \\ \text { Distilled Water } & 1000 \mathrm{ml}\end{array}$

Peptone was added to distilled water in a $1000 \mathrm{ml}$ flask and stirred for 5 minutes. Nine $\mathrm{ml}$ of solution was dispensed by pipette into test tubes, capped and autoclaved for 25 minutes. Tubes were allowed to cool for 1 hour prior to use.

\section{Appendix C: dsRNA Extraction Protocol}

Cellophane (Flexal Corp.) is cut to the diameter of 100x15 mm Petri plates (Fisherbrand Scientific), added to a glass Petri dish containing distilled water, covered and autoclaved for 24 minutes at 15 psi. The cellophane is then aseptically transferred to PDA plates amended with tetracycline hydrochloride and streptomycin sulfate. Agar plugs containing mycelium of test isolates are replicated on 5 plates. Plates are incubated at $20^{\circ} \mathrm{C}$ for $7-10$ days. The mycelium is then scraped from the cellophane into a cold mortar, immersed in liquid nitrogen and ground to a fine powder.

The mycelial powder is added to a $30 \mathrm{ml}$ polyallomer screw cap test tube, to which is added: $10 \mathrm{ml} 2 \mathrm{X}$ STE (containing 20\% Sodium dodecyl sulfate), $11 \mathrm{ml}$ phenol (containing $0.1 \%$ 8-hydroxyquinoline and $8 \mathrm{ml}$ chloroform/isoamyl alcohol (24:1). Tubes are capped, covered with ice, and placed on a rotary shaker ( 100 rpm) for 30-45 minutes. After shaking, the tubes are centrifuged for 30 minutes at $8,000 \mathrm{rpm}$ at $0-4^{\circ} \mathrm{C}$. The aqueous phase is collected in a $25 \mathrm{ml}$ graduated cylinder and the volumn brought up to $20 \mathrm{ml}$ with $1 \mathrm{X}$ STE and $95 \% \mathrm{EtOH}$ added to a final concentration of $15 \%$.

Twelve grams of chromatographic cellulose powder (Whatman CF-11 cellulose) is equilibrated with $200 \mathrm{ml} \mathrm{STE}$ : 15\% EtOH. The cellulose solution is mixed continuously and $25 \mathrm{ml}$ is added to fritted glass columns and allowed to drain. The nucleic acid sample is then applied to the column and washed with $80-100 \mathrm{ml}$ STE: $15 \%$ EtOH. Bromphenol blue solution is added to the surface of the CF-11 column (3-5 drops) then nucleic acids are eluted with $11 \mathrm{ml} 1 \mathrm{X}$ STE (no alcohol). Elute is collected in a $30 \mathrm{ml}$ glass Corex tube.

Eighteen $\mathrm{ml}$ of $95 \% \mathrm{EtOH}$ and 98 drops of sodium acetate are added to each tube. The tubes are covered with parafilm and the contents mixed thoroughly. The samples are stored at $-20^{\circ} \mathrm{C}$ overnight.

Upon removal from the freezer, the tubes are centrifuged at $8000 \mathrm{rpm}$ for 30 minutes. The supernatant is decanted and the excess alcohol is wiped from the tubes along the line of centrifugation with kimwipes. The dsRNA precipitate is resuspended with $1 \mathrm{ml}$ of the resuspension buffer and vortexed for 30 seconds. 
Traces of DNA are removed from the nucleic acid sample by adding $100 \mu \mathrm{l} 0.5 \mathrm{M}$ $\mathrm{MgCl} 2$ and 20 $\mu$ l DNAse (Promega RQ1 RNAse-free DNAse) for 60 minutes. Two ml of cold $95 \% \mathrm{EtOH}$ and 1 drop of sodium acetate are then added to each tube. The solution is vortexed and the tubes are stored at $-20^{\circ} \mathrm{C}$ for 2 hours.

Samples are spun at $14,000 \mathrm{rpm}$ and alcohol is decanted. The remaining pellet is dried and resuspended in $15 \mu$ l resuspension buffer. An agarose gel stained with ethidium bromide is immersed in 1L of 1X TBE in an electrophoresis tank. Each well is loaded with $5-10 \mu$ l of sample. The gel is electrophoresed for 2 hours at $100 \mathrm{mV}$. Gels are examined under UV light $(250 \mathrm{~nm})$ for florescent bands of dsRNA and photographed with Genesnap software (Syngene ${ }^{\circledR}$ ).

\section{Buffers}

\section{X STE:}

0.5 M Tris

$61.0 \mathrm{~g}$

0.1 M Sodium chloride $\quad 58.0 \mathrm{~g}$

0.001 M Disodium EDTA $\quad 3.7 \mathrm{~g}$

Distilled Water

$1000.0 \mathrm{ml}$

Adjust $\mathrm{pH}$ to 6.87 with glacial acetic acid

\section{X TBE:}

Tris

$54.5 \mathrm{~g}$

Boric Acid

$27.8 \mathrm{~g}$

Disodium EDTA

$1.9 \mathrm{~g}$

Distilled Water

$1000.0 \mathrm{ml}$

\section{Bromcresol Blue Solution}

Bromcresol Blue

Sucrose

Distilled Water
$50.0 \mathrm{mg}$

$25.0 \mathrm{~g}$

$50.0 \mathrm{ml}$

\section{Resuspension Buffer}

10X TBE

$2.0 \mathrm{ml}$

Sucrose

$4.0 \mathrm{~g}$

Distilled Water

$38.0 \mathrm{ml}$ 


\section{Appendix D: Tree number and placement of hypovirulent isolates}

\begin{tabular}{|c|c|c|c|c|}
\hline $\begin{array}{l}\mathrm{T} \\
\mathrm{R} \\
\mathrm{E} \\
\mathrm{E}\end{array}$ & $50 \mathrm{CM}$ & $100 \mathrm{CM}$ & $150 \mathrm{CM}$ & $200 \mathrm{CM}$ \\
\hline 1 & Schomberg(Ep713) T & Ep155(Ep713) T & Bockenhauer(Ep713) T & Euro7ssv(Ep713) A \\
\hline 2 & JR10(Ep713) T & WR2(Ep713) A & Ep155(Ep713) A & Schomberg(Ep713) A \\
\hline 3 & Ep155(Ep713) A & Euro7ssv(Ep713) T & WR2(Ep713) A & Bockenhauer(Ep713) T \\
\hline 4 & JR10(Euro7)A & Bockenhauer(Euro7) A & Schomberg(Euro7) T & Ep155(Euro7) T \\
\hline 5 & Euro7ssv(Euro7) A & WR2(Euro7) T & Ep155(Euro7) T & WR2(Euro7) A \\
\hline 6 & Schomberg(Euro7) A & Euro7ssv(Euro7) A & Ep155(Euro7) A & WR2(Euro7) A \\
\hline 7 & Bockenhauer(Ep713) T & Ep155(Ep713) T & Schomberg(Ep713) T & WR2(Ep713) T \\
\hline 8 & WR2(Euro7) A & Euro7ssv(Euro7) A & WR2(Euro7) T & JR10(Euro7) A \\
\hline 9 & Schomberg(Ep713) A & Schomberg(Ep713) T & Euro7ssv(Ep713) A & JR10(Ep713) A \\
\hline 10 & Schomberg (Euro7) A & JR10(Euro7) A & JR10(Euro7) T & Bockenhauer(Euro7) A \\
\hline 11 & WR2(Euro7) T & Bockenhauer(Euro7) T & Euro7ssv(Euro7) T & JR10(Euro7) T \\
\hline 12 & Euro7ssv(Euro7) A & Ep155(Euro7) T & WR2(Euro7) T & JR10(Euro7) A \\
\hline 13 & Schomberg(Euro7) $\mathrm{T}$ & WR2(Euro7) T & Euro7ssv(Euro7) T & JR10(Euro7) A \\
\hline 14 & WR2(Coli) & Schomberg(Coli) & Ep155(Coli) & Euro7ssv(Coli) \\
\hline 15 & Schomberg(Euro7) A & WR2(Euro7) A & WR2(Euro7) T & Schomberg (Euro7) T \\
\hline 16 & Bockenhauer(Coli) & WR2(Coli) & Schomberg(Coli) & Euro7ssv(Coli) \\
\hline 17 & Euro7ssv(Coli) & JR10(Coli) & WR2(Coli) & Schomberg(Coli) \\
\hline 18 & JR10(Ep713) A & Ep155(Ep713) T & WR2(Ep713) A & Ep155(Ep713) A \\
\hline 19 & Euro7ssv(Euro7) A & Ep155(Euro7) A & Ep155(Euro7) T & Schomberg(Euro7) T \\
\hline 20 & Euro7ssv(Ep713) T & Ep155(Ep713) A & JR10(Ep713) T & Ep155(Ep713) T \\
\hline 21 & WR2(Euro7) A & Schomberg(Euro7) A & Ep155(Euro7) A & Ep155(Euro7) T \\
\hline 22 & WR2(Euro7) T & Ep155(Euro7) A & Euro7ssv(Euro7) T & Schomberg(Euro7) T \\
\hline 23 & JR10(Euro7) T & Euro7ssv(Euro7) T & Schomberg(Euro7) A & Bockenhauer(Euro7) A \\
\hline 24 & JR10(Coli) & Ep155(Coli) & JR10(Coli) & Schomberg(Coli) \\
\hline 25 & JR10(Coli) & Bockenhauer(Coli) & Schomberg(Coli) & Euro7ssv(Coli) \\
\hline 26 & JR10(Euro7) A & Ep155(Euro7) T & WR2(Euro7) A & Schomberg(Euro7) A \\
\hline 27 & Bockenhauer(Ep713) A & JR10(Ep713) A & WR2(Ep713) A & Ep155(Ep713) T \\
\hline 28 & Bockenhauer(Ep713) T & WR2(Ep713) A & Ep155(Ep713) A & Schomberg(Ep713) T \\
\hline 29 & WR2(Ep713) A & Schomberg(Ep713) A & Bockenhauer(Ep713) A & JR10(Ep713) A \\
\hline 30 & Schomberg(Ep713) A & Bockenhauer(Ep713) A & WR2(Ep713) T & Schomberg(Ep713) T \\
\hline 31 & Euro7ssv(Ep713) A & WR2(Ep713) A & JR10(Ep713) T & JR10(Ep713) A \\
\hline 32 & Bockenhauer(Euro7) A & Schomberg(Euro7) T & Euro7ssv(Euro7) T & Schomberg(Euro7) A \\
\hline 33 & Bockenhauer(Coli) & WR2(Coli) & Bockenhauer(Coli) & Ep155(Coli) \\
\hline 34 & Ep155(Ep713) T & JR10(Ep713) A & Schomberg(Ep713) A & Euro7ssv(Ep713) A \\
\hline 35 & WR2(Coli) & Bockenhauer(Coli) & JR10(Coli) & Ep155(Coli) \\
\hline 36 & WR2(Euro7) T & WR2(Euro7) A & JR10(Euro7) A & Bockenhauer(Euro7) T \\
\hline 37 & Ep155(Euro7) A & Bockenhauer(Euro7) A & Bockenhauer(Euro7) T & JR10(Euro7) T \\
\hline 38 & Ep155(Ep713) A & Schomberg(Ep713) A & Bockenhauer(Ep713) A & Euro7ssv(Ep713) T \\
\hline 39 & Bockenhauer(Euro7) T & JR10(Euro7) T & JR10(Euro7) A & Ep155(Euro7) A \\
\hline 40 & Bockenhauer(Ep713) T & JR10(Ep713) T & Euro7ssv(Ep713) T & Euro7ssv(Ep713) A \\
\hline 41 & Euro7ssv(Ep713) T & Schomberg(Ep713) T & JR10(Ep713) T & Bockenhauer(Ep713) T \\
\hline 42 & WR2(Ep713) T & Euro7ssv(Ep713) T & JR10(Ep713) T & Bockenhauer(Ep713) A \\
\hline 43 & Euro7ssv(Ep713) T & Schomberg(Ep713) T & Bockenhauer(Ep713) T & Ep155(Ep713) A \\
\hline 44 & Ep155(Euro7) T & Schomberg(Euro7) T & Schomberg(Euro7) A & Euro7ssv(Euro7) T \\
\hline 45 & JR10(Ep713) A & Ep155(Ep713) T & Bockenhauer(Ep713) A & WR2(Ep713) T \\
\hline 46 & Bockenhauer(Ep713) A & JR10(Ep713) A & WR2(Ep713) T & Euro7ssv(Ep713) T \\
\hline 47 & Schomberg(Coli) & Ep155(Coli) & JR10(Coli) & Ep155(Coli) \\
\hline 48 & Ep155(Coli) & WR2(Coli) & Bockenhauer(Coli) & Euro7ssv(Coli) \\
\hline 49 & Euro7ssv(Ep713) A & Bockenhauer(Ep713) T & Schomberg(Ep713)T & WR2(Ep713) T \\
\hline 50 & Euro7ssv(Coli) & WR2(Coli) & Bockenhauer(Coli) & Schomberg(Coli) \\
\hline 51 & JR10(Euro7) T & WR2(Euro7) A & Bockenhauer(Euro7) T & Bockenhauer(Euro7) A \\
\hline 52 & Bockenhauer(Ep713) A & WR2(Ep713) T & Ep155(EP713) T & JR10(Ep713) T \\
\hline 53 & Ep155(Euro7) A & Bockenhauer(Euro7) A & Ep155(Euro7) T & Schomberg(Euro7) T \\
\hline
\end{tabular}




\begin{tabular}{|l|l|l|l|l|}
\hline 54 & JR10(Coli) & WR(Coli) & Bockenhauer(Coli) & Euro7ssv(Coli) \\
\hline 55 & Ep155(Euro7) A & Euro7ssv(Euro7) T & Bockenhauer(Euro7) T & Euro7ssv(Euro7) A \\
\hline 56 & Euro7ssv(Ep713) A & JR10(Ep713) T & Schomberg(Ep713) A & WR2(Ep713) T \\
\hline 57 & WR2(Ep713) A & Schomberg(Ep713) A & Ep155(Ep713) A & Euro7ssv(Ep713) A \\
\hline 58 & Bockenhauer(Euro7) A & Bockenhauer(Euro7) T & Euro7ssv(Euro7) A & JR10(Euro7) T \\
\hline 59 & JR10(Coli) & Euro7ssv(Coli) & Ep155(Coli) & Schomberg(Coli) \\
\hline 60 & Euro7ssv(Euro7) A & JR10(Euro7) T & Euro7ssv(Euro7) T & Bockenhauer(Euro7) T \\
\hline 88 & Schomberg(Coli) & Bockenhauer(Coli) & JR10(Coli) & WR2(Coli) \\
\hline 89 & Bockenhauer(Euro7)T & Schomberg(Euro7)A & Bockenhauer(Euro7)A & Schomberg(Euro7)T \\
\hline 90 & Bockenhauer(Ep713)A & Schomberg(Ep713)T & Bockenhauer(Ep713)T & Schomberg(Ep713)A \\
\hline 91 & WR2(Euro7)A & JR10(Euro7)A & JR10(Euro7)T & WR2(Euro7)T \\
\hline 92 & WR2(Ep713)A & JR10(Ep713)T & WR2(Ep713)T & JR10(Ep713)A \\
\hline 93 & Ep155(Coli) & Euro7ssv(Coli) & & \\
\hline 94 & Ep155(Euro7)A & Ep155(Euro7)T & Euro7ssv(Euro7)A & Euro7ssv(Euro7)T \\
\hline 95 & Ep155(Ep713)A & Ep155(Ep713)T & Euro7ssv(Ep713)A & Euro7ssv(Ep713)T \\
\hline
\end{tabular}

\section{Virulent Isolate placement}

\begin{tabular}{|l|l|l|}
\hline TREE & $\mathbf{5 0}$ CM & $\mathbf{1 5 0 C M}$ \\
\hline 61 & WR2 & JR10 \\
\hline 62 & WR2 & Euro7ssv \\
\hline 63 & Ep155 & Bockenhauer \\
\hline 64 & Bockenhauer & Euro7ssv \\
\hline 65 & Ep155 & WR2 \\
\hline 66 & Euro7ssv & Schomberg \\
\hline 67 & WR2 & Ep155 \\
\hline 68 & Schomberg & Bockenhauer \\
\hline 69 & Ep155 & JR10 \\
\hline 70 & WR2 & JR10 \\
\hline 71 & Schomberg & WR2 \\
\hline 72 & Bockenhauer & JR10 \\
\hline 73 & JR10 & \\
\hline
\end{tabular}




\begin{tabular}{|l|l|l|}
\hline 74 & Ep155 & Euro7ssv \\
\hline 75 & Ep155 & Bockenhauer \\
\hline 76 & JR10 & WR2 \\
\hline 78 & Bockenhauer & JR10 \\
\hline 79 & WR2 & Ep155 \\
\hline 80 & Schomberg & Ep155 \\
\hline 81 & Euro7ssv & Schomberg \\
\hline 82 & Schomberg & Euro7ssv \\
\hline 83 & Bockenhauer & Euro7ssv \\
\hline 84 & JR10 & Bockenhauer \\
\hline 85 & Euro7ssv & Schomberg \\
\hline 86 & JR10 & WR2 \\
\hline 87 & Bockenhauer & Schomberg \\
\hline
\end{tabular}




\section{Appendix E: Histograms Illustrating Canker Recovery}

\section{Cankers Initiated by Bockenhauer Isolates}

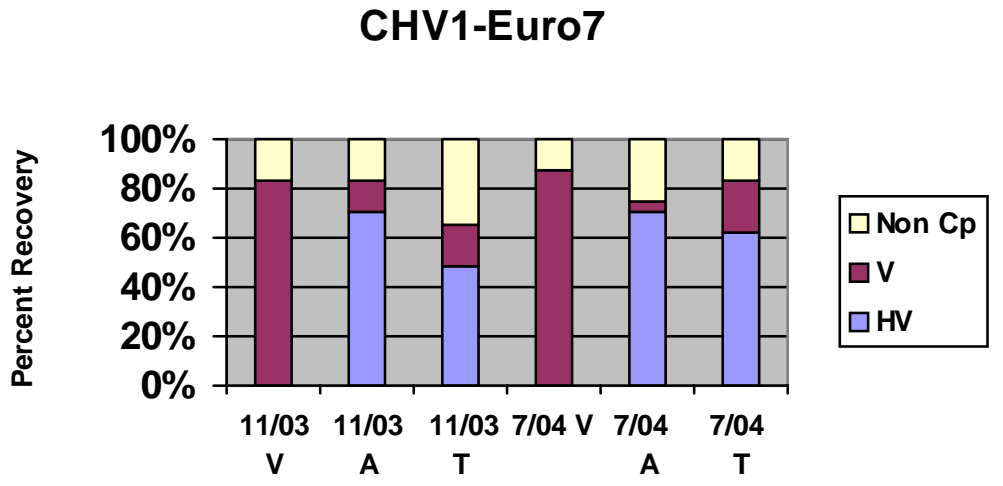

\section{CHV1-Ep713}

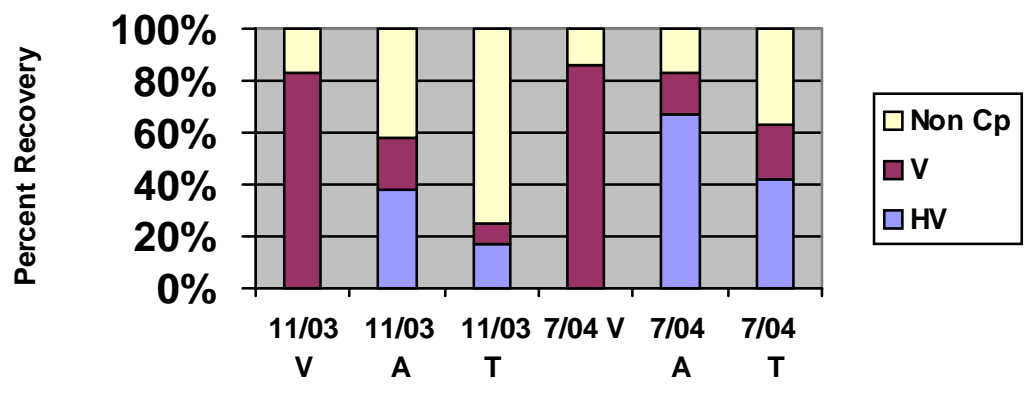

\section{CHV3-County Line}

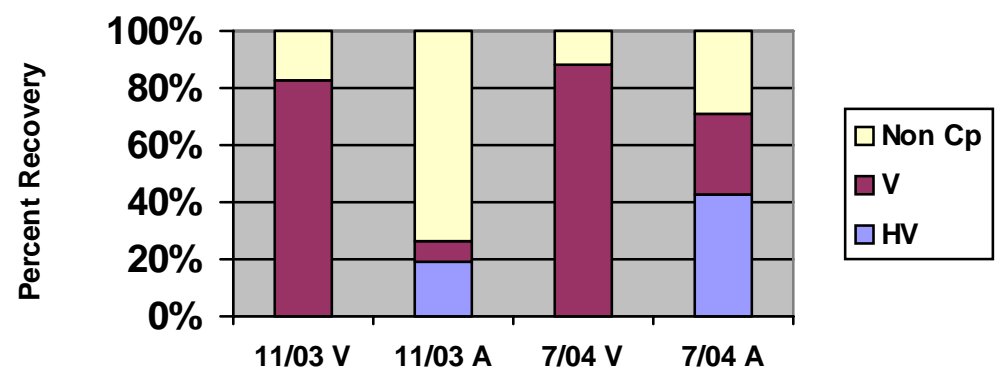

Figure 23. Histograms illustrating the percentage of hypovirulent (HV), virulent (V), and non-C. parastica (Non Cp) isolates recovered from cankers initiated by all Bockenhauer isolates sampled after 5 (11/03) and 12 months (7/04). A= anastomosed isolates. $\mathrm{T}=$ transfected isolates. $\mathrm{V}=$ virulent isolates. 


\section{Cankers Initiated by Schomberg Isolates}

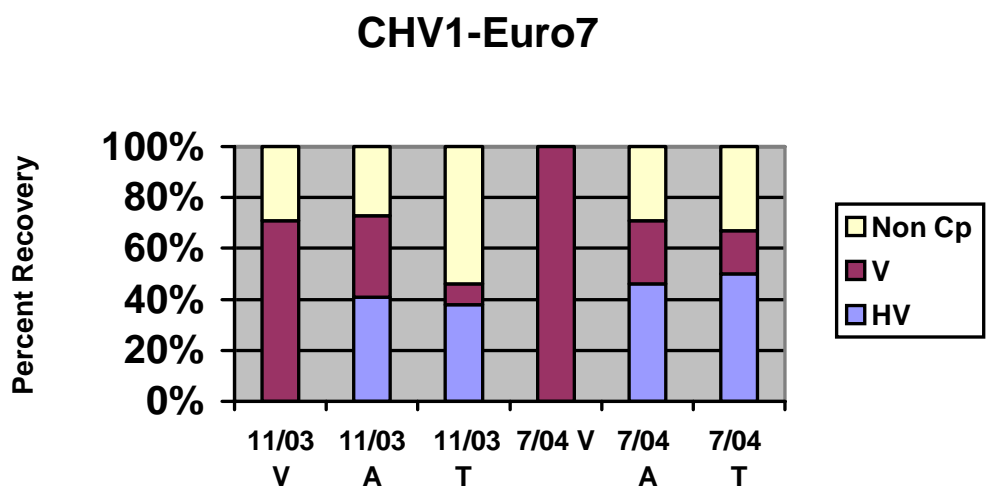

\section{CHV1-Ep713}

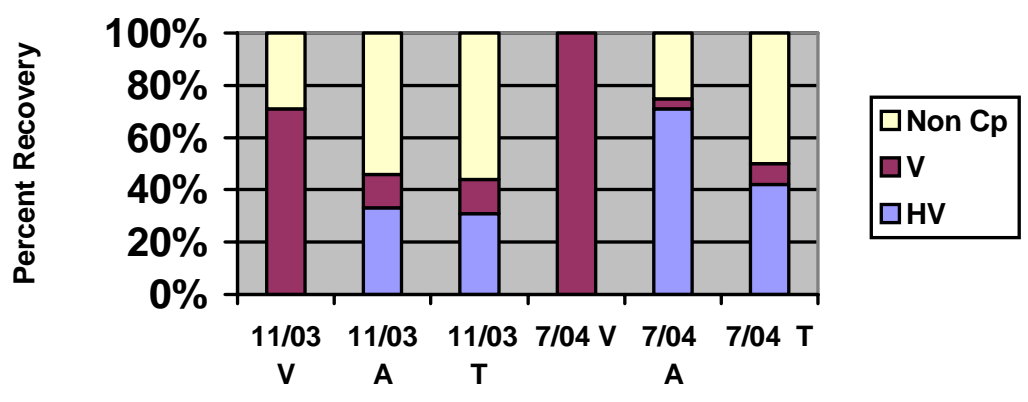

CHV3-County Line

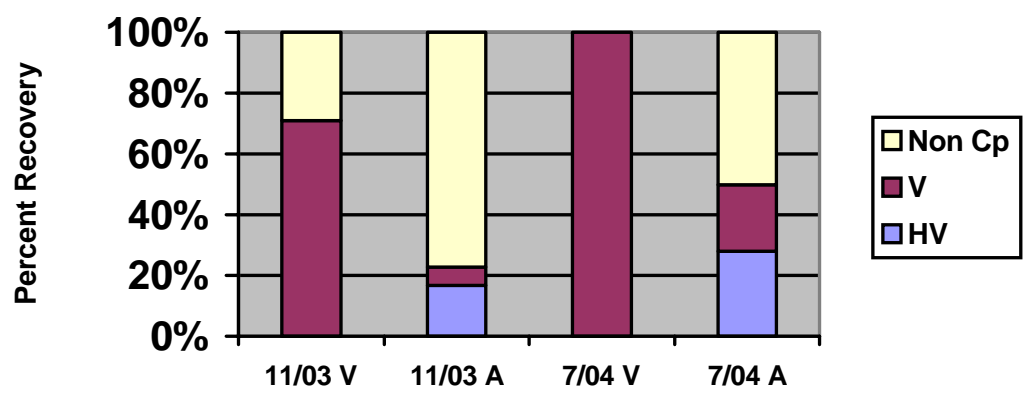

Figure 24. Histograms illustrating the percentage of hypovirulent (HV), virulent (V), and non-C. parastica (Non Cp) isolates recovered from cankers initiated by all Schomberg isolates sampled after 5 (11/03) and 12 months (7/04). $A=$ anastomosed isolates. $\mathrm{T}=$ transfected isolates. $\mathrm{V}=$ virulent isolates. 


\section{Cankers Initiated by Euro7ssv Isolates}

\section{CHV3-County Line}

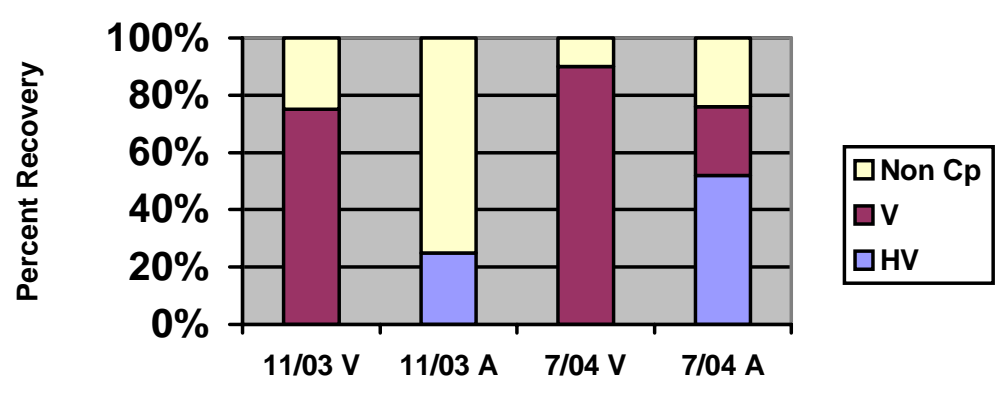

\section{CHV1-Ep713}

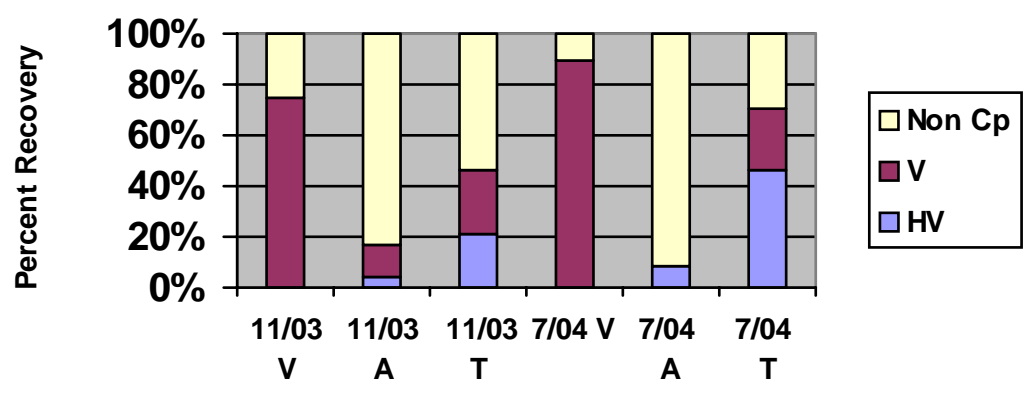

CHV3-County Line

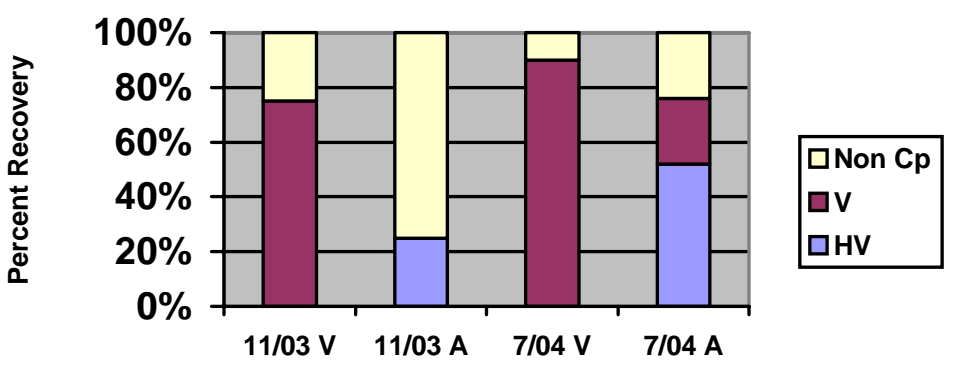

Figure 25. Histograms illustrating the percentage of hypovirulent (HV), virulent (V), and non-C. parastica (Non Cp) isolates recovered from cankers initiated by all Euro7ssv isolates sampled after 5 (11/03) and 12 months (7/04). A= anastomosed isolates. $\mathrm{T}=$ transfected isolates. $\mathrm{V}=$ virulent isolates. 


\section{Cankers Initiated by JR10 Isolates}

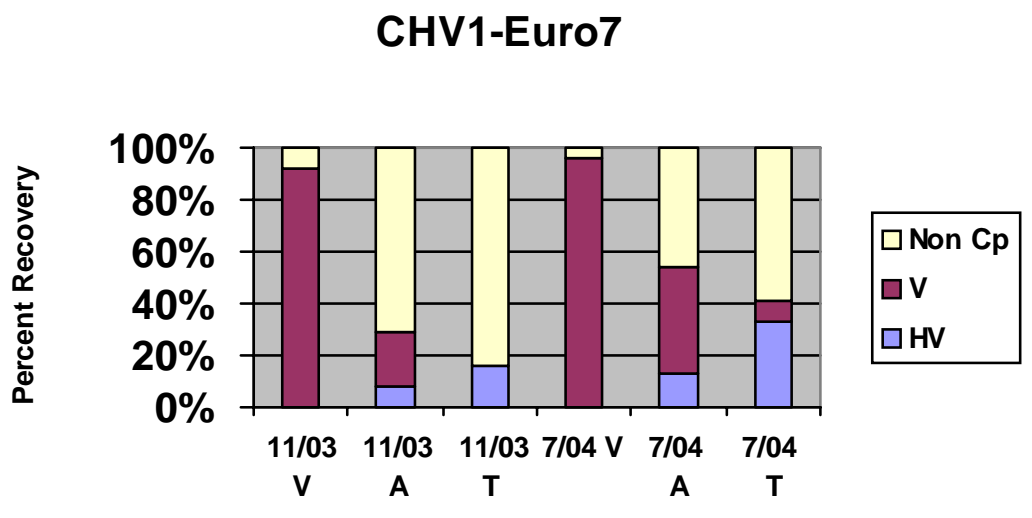

\section{CHV1-Ep713}

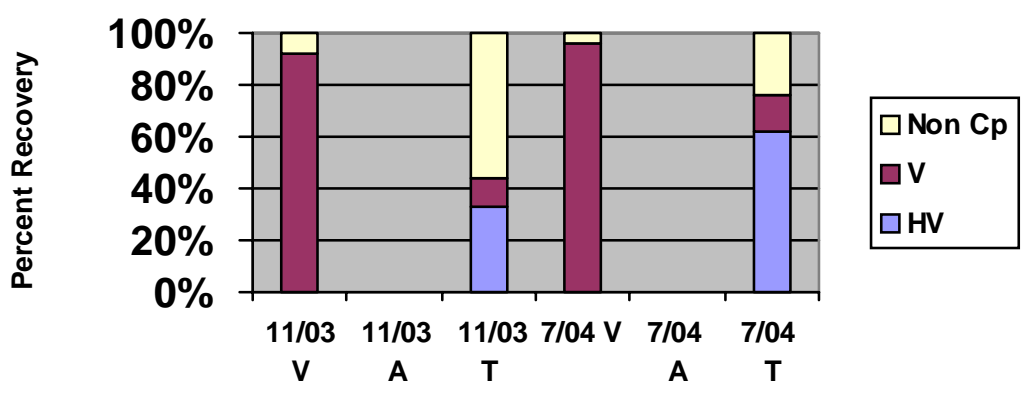

CHV3-County Line

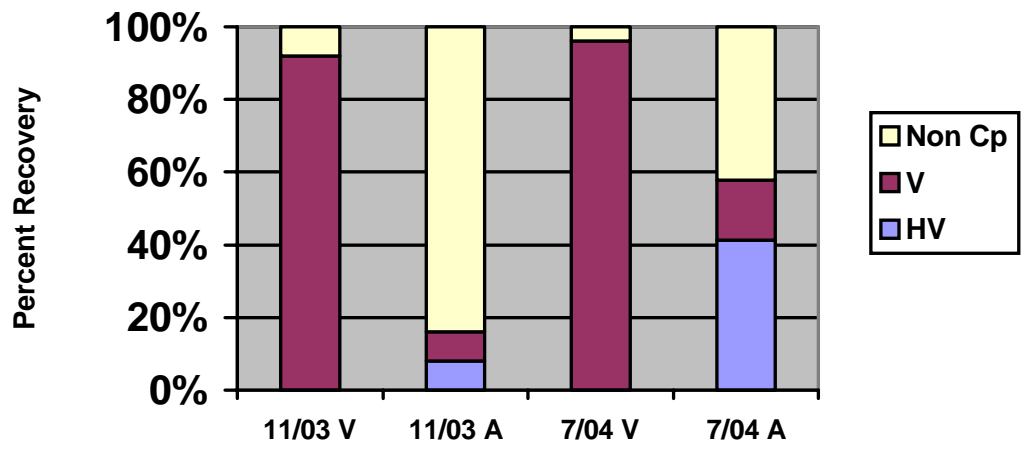

Figure 26. Histograms illustrating the percentage of hypovirulent (HV), virulent (V), and non-C. parastica (Non Cp) isolates recovered from cankers initiated by all JR10 isolates sampled after 5 (11/03) and 12 months (7/04). A= anastomosed isolates. $\mathrm{T}=$ transfected isolates. $\mathrm{V}=$ virulent isolates. 


\section{Cankers Initiated by WR2 Isolates}

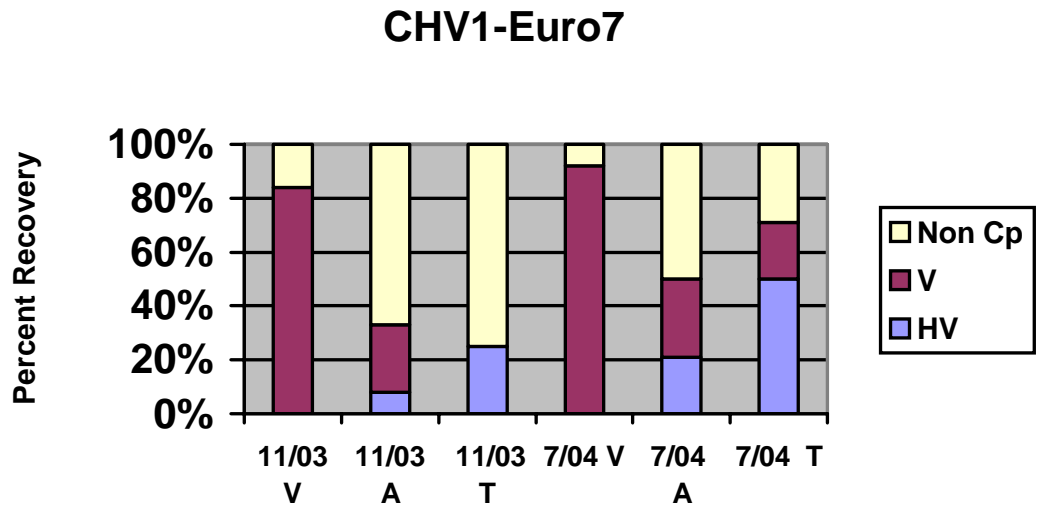

\section{CHV3-County Line}

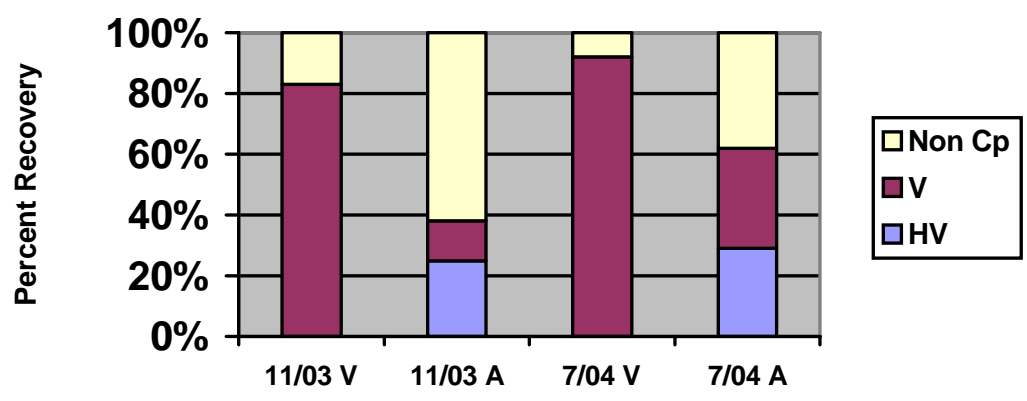

CHV3-County Line

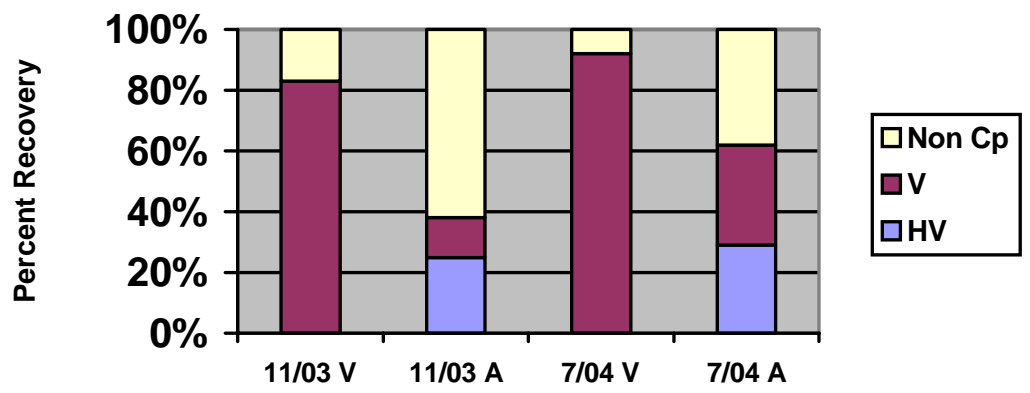

Figure 27. Histograms illustrating the percentage of hypovirulent (HV), virulent (V), and non-C. parastica (Non Cp) isolates recovered from cankers initiated by all WR2 isolates sampled after $5(11 / 03)$ and 12 months $(7 / 04) . A=$ anastomosed isolates. $\mathrm{T}=$ transfected isolates. $\mathrm{V}=$ virulent isolates. 


\section{Cankers Initiated by Ep155 Isolates}

\section{CHV1-Ep713}

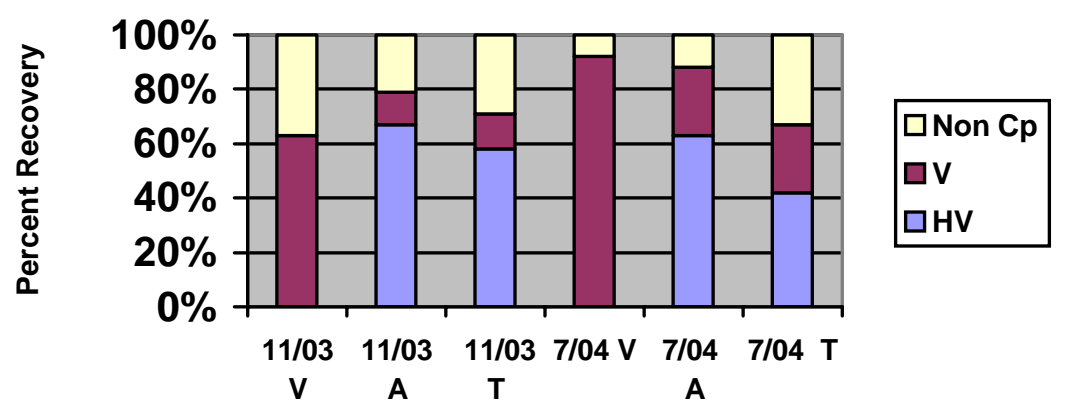

CHV3-County Line

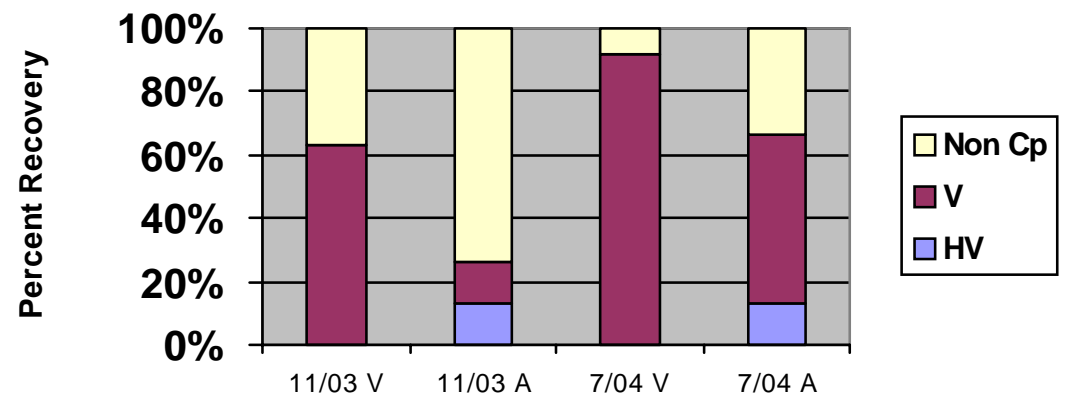

Figure 28. Histograms illustrating the percentage of hypovirulent (HV), virulent (V), and non-C. parastica (Non Cp) isolates recovered from cankers initiated by all Ep155 isolates sampled after 5 (11/03) and 12 months (7/04). A= anastomosed isolates. $\mathrm{T}=$ transfected isolates. $\mathrm{V}=$ virulent isolates. 


\section{Appendix F: ANOVA Tables}

One-way ANOVA evaluated virulent (virus-free) isolates and isolates that acquired the hypoviruses by either anastomosis or transfection by using a 1x3 factorial design. LSMeans contrasts were used to further determine where significant differences occurred.

Canker Growth after 4 Months

Analysis of Variance

\begin{tabular}{|l|l|l|l|l|}
\hline Source & DF & Sum of Squares & Mean Square & F Ratio \\
\hline Model & 2 & 495.563807 & 247.781904 & 177.8851 \\
\hline Error & 200 & 278.586439 & 1.39293219 & Prob $>$ F \\
\hline C. Total & 202 & 774.150246 &. & $<.0001$ \\
\hline
\end{tabular}

\section{Effect Tests}

\begin{tabular}{|l|l|l|l|l|l|}
\hline Source & $\begin{array}{l}\text { N. } \\
\text { Parm. }\end{array}$ & DF & $\begin{array}{l}\text { Sum of } \\
\text { Squares }\end{array}$ & F Ratio & Prob $>$ F \\
\hline method of infection & 2 & 2 & 495.563807 & 177.885115 & $<.0001$ \\
\hline
\end{tabular}

LSMeans contrasts between anastomosed and transfected isolates

\begin{tabular}{|l|l|}
\hline Sum of Squares & 0.86893744 \\
\hline Numerator DF & 1 \\
\hline Denominator DF & 200 \\
\hline F Ratio & 0.62381891 \\
\hline Prob $>$ F & 0.4305671 \\
\hline
\end{tabular}

$\underline{\text { Canker Growth after } 12 \text { Months }}$

Analysis of Variance

\begin{tabular}{|l|l|l|l|l|}
\hline Source & DF & Sum of Squares & Mean Square & F Ratio \\
\hline Model & 2 & 8130.93486 & 4065.46743 & 1525.642 \\
\hline Error & 200 & 532.951843 & 2.66475922 & Prob $>$ F \\
\hline C. Total & 202 & 8663.8867 &. & $<.0001$ \\
\hline
\end{tabular}

\section{Effect Tests}

\begin{tabular}{|l|l|l|l|l|l|}
\hline Source & $\begin{array}{l}\text { N. } \\
\text { Parm. }\end{array}$ & DF & $\begin{array}{l}\text { Sum of } \\
\text { Squares }\end{array}$ & F Ratio & Prob $>$ F \\
\hline method of infection & 2 & 2 & 8130.93486 & 1525.64157 & $<.0001$ \\
\hline
\end{tabular}


LSMeans contrasts between anastomosed and transfected isolates

\begin{tabular}{|l|l|}
\hline Sum of Squares & 3.59493776 \\
\hline Numerator DF & 1 \\
\hline Denominator DF & 200 \\
\hline F Ratio & 1.34906664 \\
\hline Prob $>$ F & 0.24682566 \\
\hline
\end{tabular}

$\underline{\text { HV recovery after } 4 \text { Months }}$

Analysis of Variance

\begin{tabular}{|l|l|l|l|l|}
\hline Source & DF & Sum of Squares & Mean Square & F Ratio \\
\hline Model & 2 & 3.54483794 & 1.77241897 & 20.7721 \\
\hline Error & 201 & 17.1506958 & 0.08532684 & Prob $>$ F \\
\hline C. Total & 203 & 20.6955338 & & $<.0001$ \\
\hline
\end{tabular}

\section{Effect Tests}

\begin{tabular}{|l|l|l|l|l|l|}
\hline Source & $\begin{array}{l}\text { N. } \\
\text { Parm. }\end{array}$ & DF & $\begin{array}{l}\text { Sum of } \\
\text { Squares }\end{array}$ & F Ratio & Prob $>$ F \\
\hline method of infection & 2 & 2 & 3.54483794 & 20.7721142 & $<.0001$ \\
\hline
\end{tabular}

LSMeans contrasts between anastomosed and transfected isolates

\begin{tabular}{|l|l|}
\hline Sum of Squares & 0.02385614 \\
\hline Numerator DF & 1 \\
\hline Denominator DF & 201 \\
\hline F Ratio & 0.27958539 \\
\hline Prob $>$ F & 0.59755648 \\
\hline
\end{tabular}

$\underline{\text { HV recovery after } 12 \text { Months }}$

Analysis of Variance

\begin{tabular}{|l|l|l|l|l|}
\hline Source & DF & Sum of Squares & Mean Square & F Ratio \\
\hline Model & 2 & 6.96753309 & 3.48376655 & 36.1839 \\
\hline Error & 201 & 19.3521837 & 0.09627952 & Prob $>$ F \\
\hline C. Total & 203 & 26.3197168 & & $<.0001$ \\
\hline
\end{tabular}

\section{Effect Tests}

\begin{tabular}{|l|l|l|l|l|l|}
\hline Source & $\begin{array}{l}\text { N. } \\
\text { Parm. }\end{array}$ & DF & $\begin{array}{l}\text { Sum of } \\
\text { Squares }\end{array}$ & F Ratio & Prob $>$ F \\
\hline method of infection & 2 & 2 & 6.96753309 & 36.1838792 & $<.0001$ \\
\hline
\end{tabular}


LSMeans contrasts between anastomosed and transfected isolates

\begin{tabular}{|l|l|}
\hline Sum of Squares & 0.33086475 \\
\hline Numerator DF & 1 \\
\hline Denominator DF & 201 \\
\hline F Ratio & 3.43650186 \\
\hline Prob $>$ F & 0.06523589 \\
\hline
\end{tabular}

Hypovirus Transmission to Conidia

Analysis of Variance

\begin{tabular}{|l|l|l|l|l|}
\hline Source & DF & Sum of Squares & Mean Square & F Ratio \\
\hline Model & 2 & 10.2155991 & 5.10779955 & 129.9049 \\
\hline Error & 75 & 2.94896471 & 0.03931953 & Prob $>$ F \\
\hline C. Total & 77 & 13.1645638 &. & $<.0001$ \\
\hline
\end{tabular}

Effect Tests

\begin{tabular}{|l|l|l|l|l|l|}
\hline Source & $\begin{array}{l}\text { N. } \\
\text { Parm. }\end{array}$ & DF & $\begin{array}{l}\text { Sum of } \\
\text { Squares }\end{array}$ & F Ratio & Prob $>$ F \\
\hline method of infection & 2 & 2 & 10.2155991 & 129.9049 & $<.0001$ \\
\hline
\end{tabular}

LSMeans contrasts between anastomosed and transfected isolates

\begin{tabular}{|l|l|}
\hline Sum of Squares & 0.0317095 \\
\hline Numerator DF & 1 \\
\hline Denominator DF & 75 \\
\hline F Ratio & 0.80645668 \\
\hline Prob $>$ F & 0.37204382 \\
\hline
\end{tabular}

Three-way ANOVA evaluated fungal isolates, method of infection, and hypoviruses using a 2x2x6 nested factorial design (virulent isolates were not included).

Canker Growth after 4 Months

Analysis of Variance

\begin{tabular}{|l|l|l|l|l|}
\hline Source & DF & Sum of Squares & Mean Square & F Ratio \\
\hline Model & 19 & 128.373159 & 6.75648206 & 12.9764964 \\
\hline Error & 135 & 70.2905506 & 0.52067075 & Prob $>$ F \\
\hline C. Total & 154 & 198.66371 &. & $<.0001$ \\
\hline
\end{tabular}


Effect Tests

\begin{tabular}{|l|l|l|l|l|l|}
\hline Source & $\begin{array}{l}\text { N. } \\
\text { Parm. }\end{array}$ & DF & $\begin{array}{l}\text { Sum of } \\
\text { Squares }\end{array}$ & F Ratio & Prob $>$ F \\
\hline isolate & 5 & 5 & 56.0433932 & 21.5273832 & $<.0001$ \\
\hline virus[isolate] & 5 & 5 & 33.7839413 & 12.9770845 & $<.0001$ \\
\hline method[isolate,virus] & 9 & 9 & 28.3457961 & 6.04899148 & $<.0001$ \\
\hline
\end{tabular}

Canker Growth after 12 months

Analysis of Variance

\begin{tabular}{|l|l|l|l|l|}
\hline Source & DF & Sum of Squares & Mean Square & F Ratio \\
\hline Model & 19 & 134.262935 & 7.06647026 & 9.3436 \\
\hline Error & 136 & 102.855655 & 0.75629158 & Prob $>$ F \\
\hline C. Total & 155 & 237.11859 &. & $<.0001$ \\
\hline
\end{tabular}

\section{Effect Tests}

\begin{tabular}{|l|l|l|l|l|l|}
\hline Source & $\begin{array}{l}\text { N. } \\
\text { Parm. }\end{array}$ & DF & $\begin{array}{l}\text { Sum of } \\
\text { Squares }\end{array}$ & F Ratio & Prob>F \\
\hline isolate & 5 & 5 & 47.5808953 & 12.5826855 & $<.0001$ \\
\hline virus[isolate] & 5 & 5 & 52.5629019 & 13.9001685 & $<.0001$ \\
\hline method[isolate,virus] & 9 & 9 & 28.6488095 & 4.20896007 & $<.0001$ \\
\hline
\end{tabular}

HV Recovery after 4 Months

Analysis of Variance

\begin{tabular}{|l|l|l|l|l|}
\hline Source & DF & Sum of Squares & Mean Square & F Ratio \\
\hline Model & 19 & 5.93943293 & 0.31260173 & 3.7562 \\
\hline Error & 135 & 11.2351191 & 0.0832231 & Prob $>$ F \\
\hline C. Total & 154 & 17.174552 & & $<.0001$ \\
\hline
\end{tabular}

\section{Effect Tests}

\begin{tabular}{|l|l|l|l|l|l|}
\hline Source & $\begin{array}{l}\text { N. } \\
\text { Parm. }\end{array}$ & DF & $\begin{array}{l}\text { Sum of } \\
\text { Squares }\end{array}$ & F Ratio & Prob>F \\
\hline isolate & 5 & 5 & 3.50536743 & 8.42402472 & $<.0001$ \\
\hline virus[isolate] & 5 & 5 & 1.1700346 & 2.81180236 & 0.0190 \\
\hline method[isolate,virus] & 9 & 9 & 0.90525794 & 1.20860927 & 0.2947 \\
\hline
\end{tabular}


$\underline{\text { HV Recovery after } 12 \text { Months }}$

Analysis of Variance

\begin{tabular}{|l|l|l|l|l|}
\hline Source & DF & Sum of Squares & Mean Square & F Ratio \\
\hline Model & 19 & 6.16982092 & 0.32472742 & 3.2681 \\
\hline Error & 136 & 13.5132275 & 0.09936197 & Prob $>$ F \\
\hline C. Total & 155 & 19.6830484 &. & $<.0001$ \\
\hline
\end{tabular}

Effect Tests

\begin{tabular}{|l|l|l|l|l|l|}
\hline Source & $\begin{array}{l}\text { N. } \\
\text { Parm. }\end{array}$ & DF & $\begin{array}{l}\text { Sum of } \\
\text { Squares }\end{array}$ & F Ratio & Prob $>$ F \\
\hline isolate & 5 & 5 & 2.66885 & 5.37197497 & $<.0002$ \\
\hline virus[isolate] & 5 & 5 & 1.32558705 & 2.66819809 & 0.0247 \\
\hline method[isolate,virus] & 9 & 9 & 2.19510582 & 2.45466806 & 0.0127 \\
\hline
\end{tabular}

One-way ANOVA with 26 treatment combinations compared isogenic anastomosed and transfected isolates using LSMeans contrasts.

$\underline{\text { Canker Growth after } 4 \text { months }}$

Analysis of Variance

\begin{tabular}{|l|l|l|l|l|}
\hline Source & DF & Sum of Squares & Mean Square & F Ratio \\
\hline Model & 25 & 647.250321 & 25.8900128 & 36.1114 \\
\hline Error & 177 & 126.899926 & 0.71694873 & Prob $>$ F \\
\hline C. Total & 202 & 774.150246 & & $<.0001$ \\
\hline
\end{tabular}

Effect Tests

\begin{tabular}{|l|l|l|l|l|l|}
\hline Source & $\begin{array}{l}\text { N. } \\
\text { Parm. }\end{array}$ & DF & $\begin{array}{l}\text { Sum of } \\
\text { Squares }\end{array}$ & F Ratio & Prob $>$ F \\
\hline isolate & 25 & 25 & 647.250321 & 36.1113866 & $<.0001$ \\
\hline
\end{tabular}

Canker Growth after 12 months

Analysis of Variance

\begin{tabular}{|l|l|l|l|l|}
\hline Source & DF & Sum of Squares & Mean Square & F Ratio \\
\hline Model & 25 & 8347.27546 & 333.891019 & 186.6602 \\
\hline Error & 177 & 316.611235 & 1.78876404 & Prob $>$ F \\
\hline C. Total & 202 & 8663.8867 & & $<.0001$ \\
\hline
\end{tabular}


Effect Tests

\begin{tabular}{|l|l|l|l|l|l|}
\hline Source & $\begin{array}{l}\text { N. } \\
\text { Parm. }\end{array}$ & DF & $\begin{array}{l}\text { Sum of } \\
\text { Squares }\end{array}$ & F Ratio & Prob $>$ F \\
\hline isolate & 25 & 25 & 8347.27546 & 186.66018 & $<.0001$ \\
\hline
\end{tabular}

$\underline{\text { HV Recovery after } 4 \text { months }}$

Analysis of Variance

\begin{tabular}{|l|l|l|l|l|}
\hline Source & DF & Sum of Squares & Mean Square & F Ratio \\
\hline Model & 25 & 9.46041472 & 0.37841659 & 5.9953 \\
\hline Error & 178 & 11.2351191 & 0.06311865 & Prob $>$ F \\
\hline C. Total & 203 & 20.6955338 &. & $<.0001$ \\
\hline
\end{tabular}

\section{Effect Tests}

\begin{tabular}{|l|l|l|l|l|l|}
\hline Source & $\begin{array}{l}\text { N. } \\
\text { Parm. }\end{array}$ & DF & $\begin{array}{l}\text { Sum of } \\
\text { Squares }\end{array}$ & F Ratio & Prob>F \\
\hline isolate & 25 & 25 & 9.46041472 & 5.99532168 & $<.0001$ \\
\hline
\end{tabular}

HV Recovery after 12 months

Analysis of Variance

\begin{tabular}{|l|l|l|l|l|}
\hline Source & DF & Sum of Squares & Mean Square & F Ratio \\
\hline Model & 25 & 12.8064893 & 0.51225957 & 6.7476 \\
\hline Error & 178 & 13.5132275 & 0.07591701 & Prob $>$ F \\
\hline C. Total & 203 & 26.3197168 & & $<.0001$ \\
\hline
\end{tabular}

\section{Effect Tests}

\begin{tabular}{|l|l|l|l|l|l|}
\hline Source & $\begin{array}{l}\text { N. } \\
\text { Parm. }\end{array}$ & DF & $\begin{array}{l}\text { Sum of } \\
\text { Squares }\end{array}$ & F Ratio & Prob $>$ F \\
\hline isolate & 25 & 25 & 12.8064893 & 6.74762587 & $<.0001$ \\
\hline
\end{tabular}


Two-way ANOVA assessed virulent, CHV3-County Line, CHV1-Euro7, and CHV1Ep713 isolates that acquired the hypovirus by anastomosis by using a $2 \times 4$ nested factorial design.

$\underline{\text { Canker Growth after } 4 \text { Months }}$

Analysis of Variance

\begin{tabular}{|l|l|l|l|l|}
\hline Source & DF & Sum of Squares & Mean Square & F Ratio \\
\hline Model & 15 & 124.300223 & 8.28668155 & 16.8298 \\
\hline Error & 104 & 51.2075893 & 0.49238067 & Prob $>$ F \\
\hline C. Total & 119 & 175.507813 & & $<.0001$ \\
\hline
\end{tabular}

Effect Tests

\begin{tabular}{|l|l|l|l|l|l|}
\hline Source & $\begin{array}{l}\text { N. } \\
\text { Parm. }\end{array}$ & DF & $\begin{array}{l}\text { Sum of } \\
\text { Squares }\end{array}$ & F Ratio & Prob $>$ F \\
\hline isolate & 5 & 5 & 41.466455 & 16.8432507 & $<.0001$ \\
\hline virus[isolate] & 10 & 10 & 73.1138452 & 14.8490488 & $<.0001$ \\
\hline
\end{tabular}

$\underline{\text { Canker Growth after } 12 \text { Months }}$

Analysis of Variance

\begin{tabular}{|l|l|l|l|l|}
\hline Source & DF & Sum of Squares & Mean Square & F Ratio \\
\hline Model & 15 & 121.74091 & 8.11606069 & 9.6358 \\
\hline Error & 106 & 89.2821429 & 0.84228437 & Prob $>$ F \\
\hline C. Total & 121 & 211.023053 &. & $<.0001$ \\
\hline
\end{tabular}

Effect Tests

\begin{tabular}{|l|l|l|l|l|l|}
\hline Source & $\begin{array}{l}\text { N. } \\
\text { Parm. }\end{array}$ & DF & $\begin{array}{l}\text { Sum of } \\
\text { Squares }\end{array}$ & F Ratio & Prob $>$ F \\
\hline isolate & 5 & 5 & 28.8046486 & 6.83964935 & $<.0001$ \\
\hline virus[isolate] & 10 & 10 & 85.2542632 & 10.121791 & $<.0001$ \\
\hline
\end{tabular}

$\underline{\text { HV Recovery after } 4 \text { Months }}$

Analysis of Variance

\begin{tabular}{|l|l|l|l|l|}
\hline Source & DF & Sum of Squares & Mean Square & F Ratio \\
\hline Model & 15 & 5.13086711 & 0.34205781 & 4.0473 \\
\hline Error & 105 & 8.14920635 & 0.07761149 & Prob $>$ F \\
\hline C. Total & 120 & 13.2800735 & & $<.0001$ \\
\hline
\end{tabular}


Effect Tests

\begin{tabular}{|l|l|l|l|l|l|}
\hline Source & $\begin{array}{l}\text { N. } \\
\text { Parm. }\end{array}$ & DF & $\begin{array}{l}\text { Sum of } \\
\text { Squares }\end{array}$ & F Ratio & Prob>F \\
\hline isolate & 5 & 5 & 2.15691648 & 5.55824016 & $<.0001$ \\
\hline virus[isolate] & 10 & 10 & 2.54231167 & 3.27568986 & .0010 \\
\hline
\end{tabular}

$\underline{\text { HV Recovery after } 12 \text { Months }}$

Analysis of Variance

\begin{tabular}{|l|l|l|l|l|}
\hline Source & DF & Sum of Squares & Mean Square & F Ratio \\
\hline Model & 15 & 5.84854498 & 0.389903 & 4.1599 \\
\hline Error & 104 & 9.74775132 & 0.09372838 & Prob $>$ F \\
\hline C. Total & 119 & 15.5962963 & & $<.0001$ \\
\hline
\end{tabular}

Effect Tests

\begin{tabular}{|l|l|l|l|l|l|}
\hline Source & $\begin{array}{l}\text { N. } \\
\text { Parm. }\end{array}$ & DF & $\begin{array}{l}\text { Sum of } \\
\text { Squares }\end{array}$ & F Ratio & Prob>F \\
\hline isolate & 5 & 5 & 2.49002953 & 5.31328842 & .0002 \\
\hline virus[isolate] & 10 & 10 & 3.11250467 & 3.32077086 & .0009 \\
\hline
\end{tabular}

$\underline{\text { Hypovirus Transmission to Conidia }}$

Analysis of Variance

\begin{tabular}{|l|l|l|l|l|}
\hline Source & DF & Sum of Squares & Mean Square & F Ratio \\
\hline Model & 15 & 5.13086711 & 0.34205781 & 4.0473 \\
\hline Error & 105 & 8.14920635 & 0.07761149 & Prob $>$ F \\
\hline C. Total & 120 & 13.2800735 & & $<.0001$ \\
\hline
\end{tabular}

\section{Effect Tests}

\begin{tabular}{|l|l|l|l|l|l|}
\hline Source & $\begin{array}{l}\text { N. } \\
\text { Parm. }\end{array}$ & DF & $\begin{array}{l}\text { Sum of } \\
\text { Squares }\end{array}$ & F Ratio & Prob>F \\
\hline isolate & 5 & 5 & 0.31802307 & 1.93378204 & 0.1069 \\
\hline virus[isolate] & 17 & 17 & 9.88659722 & 17.6814071 & $<.0001$ \\
\hline
\end{tabular}




\section{Pearson's $\chi 2$-test}

Stroma production between pooled anastomosed and transfected isolates (virulent isolates were excluded).

$\underline{\text { Stroma Production after } 4 \text { Months }}$

\begin{tabular}{|l|l|l|l|}
\hline Source & DF & -LogLike & Rsquare (U) \\
\hline Model & 4 & 1.59797517 & 0.01534902 \\
\hline Error & 135 & 102.511277 &. \\
\hline C. Total & 139 & 104.109252 &. \\
\hline N & 143 &. &. \\
\hline
\end{tabular}

\begin{tabular}{|l|l|l|}
\hline Test & ChiSquare & Prob $>$ ChiSq \\
\hline Pearson & 2.78825613 & 0.59386161 \\
\hline
\end{tabular}

$\underline{\text { Stroma Production after } 12 \text { Months }}$

\begin{tabular}{|l|l|l|l|}
\hline Source & DF & -LogLike & Rsquare (U) \\
\hline Model & 3 & 1.73569629 & 0.04697272 \\
\hline Error & 141 & 35.2154604 &. \\
\hline C. Total & 144 & 36.9511567 &. \\
\hline N & 147 &. &. \\
\hline
\end{tabular}

\begin{tabular}{|l|l|l|}
\hline Test & ChiSquare & Prob $>$ ChiSq \\
\hline Pearson & 2.692 & 0.4416 \\
\hline
\end{tabular}

Hypovirus Transmission to Vegetative Incompatible Isolates

\begin{tabular}{|l|l|l|l|}
\hline Source & DF & -LogLike & Rsquare (U) \\
\hline Model & 19 & 12.7939833 & 0.03332163 \\
\hline Error & 580 & 371.160293 &. \\
\hline C. Total & 599 & 383.954276 &. \\
\hline N & 600 &. &. \\
\hline
\end{tabular}

\begin{tabular}{|l|l|l|}
\hline Test & ChiSquare & Prob $>$ ChiSq \\
\hline Pearson & 24.5014952 & 0.1776134 \\
\hline
\end{tabular}

